\title{
ATLAS measurements of the properties of jets for boosted particle searches
}

\author{
G. Aad et al.* \\ (ATLAS Collaboration)
}

(Received 23 June 2012; published 15 October 2012)

\begin{abstract}
Measurements are presented of the properties of high transverse momentum jets, produced in protonproton collisions at a center-of-mass energy of $\sqrt{s}=7 \mathrm{TeV}$. The data correspond to an integrated luminosity of $35 \mathrm{pb}^{-1}$ and were collected with the ATLAS detector in 2010. Jet mass, width, eccentricity, planar flow and angularity are measured for jets reconstructed using the anti- $k_{t}$ algorithm with distance parameters $R=0.6$ and 1.0, with transverse momentum $p_{T}>300 \mathrm{GeV}$ and pseudorapidity $|\eta|<2$. The measurements are compared to the expectations of Monte Carlo generators that match leading-logarithmic parton showers to leading-order, or next-to-leading-order, matrix elements. The generators describe the general features of the jets, although discrepancies are observed in some distributions.
\end{abstract}

PACS numbers: $12.38 .-\mathrm{t}, 13.30 . \mathrm{Eg}$

\section{INTRODUCTION}

The high center-of-mass energy at the Large Hadron Collider (LHC) combined with the coverage and granularity of the ATLAS calorimeter provide an excellent environment to study hadronic jets. Measurements of dijet cross sections [1,2], jet shapes [3,4], jet substructure [5] and angular correlations [6,7] have already been published using the data taken by the ATLAS and CMS Collaborations in 2010.

Massive, hadronically decaying particles produced with a significant boost (such as top quarks, Higgs bosons, or new particles) will tend to have collimated decays, such that their decay products are contained within a single jet. The substructure of jets resulting from such decays is expected to result in deviations in the observables measured here for light quarks and gluons, thus providing discriminating power in heavy particle searches.

The observable jet properties presented here are mass, width, eccentricity, planar flow and angularity. All of these have been shown to be useful in Monte Carlo studies in the search for high transverse momentum $\left(p_{\mathrm{T}}\right)$, massive particles [8-14], and together they provide an important set of probes of the substructure of jets.

Three of these (mass, planar flow and angularity) have recently been measured by CDF [13] at the Tevatron. Angularities are a family of infrared-safe quantities that have characteristic distributions for two-body decays, while planar flow discriminates between two-body and many-body decays and, for large jet masses (above about $100 \mathrm{GeV}$ ), is largely independent of the jet mass. Eccentricity is a complementary observable to planar flow, with which it is highly anticorrelated. Jet width is a

*Full author list given at the end of the article.

Published by the American Physical Society under the terms of the Creative Commons Attribution 3.0 License. Further distribution of this work must maintain attribution to the author(s) and the published article's title, journal citation, and DOI. dimensionless quantity related to the jet mass and is thus expected to retain much of the discriminatory power without being as sensitive to the detector effects on energy scale and resolution that can hinder a mass measurement.

Jet substructure measurements can be particularly vulnerable to "pileup," i.e. particles produced in multiple $p p$ interactions that occur in addition to the primary interaction, within the sensitive time of the detector. These additional interactions result in diffuse, usually soft, energy deposits throughout the central region of the detector-the region of interest for the study of high- $p_{\mathrm{T}}$ jets. This additional energy deposition can be characterized by the number of reconstructed primary vertices $\left(N_{\mathrm{PV}}\right)[15,16]$, with events having a single good vertex $\left(N_{\mathrm{PV}}=1\right)$ being considered free from the effects of pileup. The 2010 ATLAS data set provides a unique opportunity to study these effects; a significant fraction of the 2010 data set comprises $N_{\mathrm{PV}}=1$ events, making this data set ideal for evaluating the effects of pileup on jet substructure measurements. This data set has an average $N_{\mathrm{PV}} \simeq 2.2$.

\section{THE ATLAS DETECTOR}

The ATLAS detector [17] at the LHC was designed to study a wide range of physics. It covers almost the entire solid angle around the collision point with layers of tracking detectors, calorimeters and muon chambers. Tracks and vertices are reconstructed with the inner detector, which consists of a silicon pixel detector, a silicon strip detector and a transition radiation tracker, all immersed in a $2 \mathrm{~T}$ axial magnetic field provided by a superconducting solenoid.

The ATLAS reference system is a Cartesian righthanded coordinate system, with the nominal collision point at the origin. The anticlockwise beam direction defines the positive $z$ axis, while the positive $x$ axis is defined as pointing from the collision point to the center of the LHC ring and the positive $y$ axis points upwards. The azimuthal angle $\phi$ is measured around the beam axis, 
and the polar angle $\theta$ is the angle measured with respect to the $z$ axis. The pseudorapidity is given by $\eta=$ $-\ln \tan (\theta / 2)$. Transverse momentum is defined relative to the beam axis.

For the measurements presented here, the highgranularity calorimeter systems are of particular importance. The ATLAS calorimeter system provides fine-grained measurements of shower energy depositions over a large range in $\eta$.

Electromagnetic calorimetry in the range $|\eta|<4.9$ is provided by liquid-argon (LAr) sampling calorimeters. This calorimeter system enables measurements of the shower energy in up to four depth segments. For the jets measured here, the transverse granularity ranges from $0.003 \times 0.10$ to $0.10 \times 0.10$ in $\Delta \eta \times \Delta \phi$, depending on depth segment and pseudorapidity.

Hadronic calorimetry in the range $|\eta|<1.7$ is provided by a steel/scintillator-tile sampling calorimeter. This system enables measurements of the shower energy deposition in three depth segments at a transverse granularity of typically $0.1 \times 0.1$. In the end caps $(|\eta|>1.5)$, LAr technology is used for the hadronic calorimeters that match the outer $\eta$ limits of the electromagnetic end-cap calorimeters. This system enables four measurements in depth of the shower energy deposition at a transverse granularity of either $0.1 \times 0.1(1.5<|\eta|<2.5)$ or $0.2 \times$ $0.2(2.5<|\eta|<3.2)$.

\section{MONTE CARLO SIMULATION}

The QCD predictions for the hadronic final state in inelastic $p p$ collisions are based on several Monte Carlo generators with different tunes.

The PYTHIA 6.423 generator [18] with the ATLAS Minimum Bias Tune 1 (AMBT1) [19] parameter set is used as the primary generator for comparisons with the data and for extracting corrections to the data for detector effects. The AMBT1 tune uses the MRST LO* [20] parton distribution function (PDF) set with leading-order (LO) perturbative QCD matrix elements for $2 \rightarrow 2$ processes and a leading-logarithmic, $p_{\mathrm{T}}$-ordered parton shower followed by fragmentation into final-state particles using a string model [21] with Lund functions [22] for light quarks and Bowler functions [23] for heavy quarks. In addition to charged particle measurements from ATLAS minimum bias data [24,25], the AMBT1 tune uses data from LEP, SPS and the Tevatron.

An additional PYTHIA tune, PERUGIA2010 [26,27], is used for comparison with AMBT1. The PERUGIA2010 tune also uses data from LEP, SPS and the Tevatron and additionally improves the description of jet shape measurements in LEP data. The CTEQ5L [28] PDF set is used. This tune of PYTHIA is used in the calculation of the systematic uncertainties on the measurements and for comparison with the data, along with the HERWIG++ 2.4 .2 generator with its default settings [29].
The more recent HERWIG++ 2.5.1 generator is included for comparison with the final measurements at particle level, as are the AUET2B tune [30,31] of PYTHIA 6.423, the POWHEG generator interfaced to this same PYTHIA tune, and the PYTHIA 8.153 generator [32] with tune 4C [27,30]. The major difference between HERWIG++ versions 2.4.2 and 2.5.1 is the inclusion of color reconnections in the latter. The POWHEG generator, which implements next-to-leadingorder (NLO) calculations within a shower Monte Carlo context [33-36], uses the CTEQ6M [28] PDF set.

Generated events are passed through the ATLAS detector simulation program [37], which is based on GEANT4 [38]. The quark-gluon string model with an additional precompound [39] is used for the fragmentation of nuclei, and the Bertini cascade model [40] is used to describe the interactions of hadrons with the nuclear medium.

Monte Carlo events are reconstructed and analyzed using the same event selection and simulated trigger as for the data. The size and position of the collision beam spot and the detailed description of detector conditions during the data-taking runs are included in the simulation.

\section{EVENT SELECTION}

Events containing pileup can be identified by the presence of more than one primary vertex in the event, herein referred to as $N_{\mathrm{PV}}>1$. Events recorded in the 2010 ATLAS data set contain an average $N_{\mathrm{PV}} \simeq 2.2$ and include a significant fraction of $N_{\mathrm{PV}}=1$ events $(\simeq 28 \%)$; these may be used for testing the pileup correction methods.

After applying data-quality requirements, the data sample corresponds to a total integrated luminosity of $35.0 \pm$ $1.1 \mathrm{pb}^{-1}[41,42]$.

\section{A. Trigger selection}

Events must pass the ATLAS first-level trigger requiring a jet (built from calorimeter towers with a granularity of $0.1 \times 0.1$ in $\Delta \eta \times \Delta \phi$ ) with transverse energy $E_{\mathrm{T}} \geq$ $95 \mathrm{GeV}$. The selection efficiency of this trigger has been found to be close to $100 \%$ for events satisfying the offline selection criteria implemented here, with a negligible dependence on jet mass [5].

\section{B. Primary vertex selection}

All events are required to have at least one good primary vertex. This is defined as a vertex with at least five tracks with $p_{\mathrm{T}}>150 \mathrm{MeV}$ and both transverse and longitudinal impact parameters consistent with the LHC beamspot $[15,16]$. The analysis presented here makes use of the full 2010 data set. The requirement of $N_{\mathrm{PV}}=1$ is applied only where derivation of pileup corrections is not possible.

\section{High- $p_{T}$ jet selection}

Jets are reconstructed from locally calibrated topological clusters [43] using the anti- $k_{t}$ algorithm [44] with 
distance parameters of $R=0.6$ and 1.0. Jets satisfying $p_{\mathrm{T}}>300 \mathrm{GeV}$ and $|\eta|<2$ are selected for analysis. Any event containing an $R=0.6$ jet with $p_{\mathrm{T}}>30 \mathrm{GeV}$ that fails to satisfy the criteria [45] designed to safeguard against jets caused by transient detector effects and beam backgrounds is excluded from this analysis.

In simulated data, jets are reconstructed from locally calibrated topological clusters to derive corrections for pileup and determine the systematic uncertainties and detector correction factors. The corrected data distributions are then compared to Monte Carlo predictions at particle level; in this case jets are reconstructed from stable particles as opposed to clusters. Particles are deemed to be stable for the purpose of jet reconstruction if their mean lifetimes are longer than $10 \mathrm{ps}$. Neutrinos and muons are excluded, just as they are for the Monte Carlo-based jet energy scale calibration that is applied to the data. This exclusion has a negligible effect on the final measurements.

The total numbers of jets in data satisfying the selection criteria detailed here are $\sim 122000 R=1.0$ jets and $\sim 87000 R=0.6$ jets; however, only the highest $p_{\mathrm{T}}$ jet in each event is selected for this analysis. The total numbers selected for analysis are $\sim 83000 R=1.0$ jets and $\sim 62000 R=0.6$ jets.

\section{SUBSTRUCTURE OBSERVABLES AND THEIR CORRELATIONS}

\section{A. Jet mass}

The jet mass $M$ is calculated from the energies and momenta of its constituents (particles or clusters) as follows:

$$
M^{2}=\left(\sum_{i} E_{i}\right)^{2}-\left(\sum_{i} \vec{p}_{i}\right)^{2},
$$

where $E_{i}$ and $\vec{p}_{i}$ are the energy and three-momentum of the $i$ th constituent. The sum is over all jet constituents in this and all subsequent summations. The standard ATLAS reconstruction procedure is followed: clusters have their masses set to zero, while Monte Carlo particles are assigned their correct masses.

\section{B. Jet width}

The jet width $W$ is defined as:

$$
W=\frac{\sum_{i} \Delta R^{i} p_{\mathrm{T}}^{i}}{\sum_{i} p_{\mathrm{T}}^{i}},
$$

where $\Delta R^{i}=\sqrt{\left(\Delta \phi_{i}\right)^{2}+\left(\Delta \eta_{i}\right)^{2}}$ is the radial distance between the jet axis and the $i$ th jet constituent and $p_{\mathrm{T}}^{i}$ is the constituent $p_{\mathrm{T}}$ with respect to the beam axis.

\section{Eccentricity}

The jet eccentricity $\mathcal{E}$ is calculated using a principal component analysis (PCA) [12]. The PCA method provides the vector which best describes the energyweighted geometrical distribution of the jet constituents in the $(\eta, \phi)$ plane. The eccentricity is used to characterize the deviation of the jet profile from a perfect circle in this plane, and is defined as

$$
\mathcal{E}=1-\frac{v_{\min }}{v_{\max }},
$$

where $v_{\max }\left(v_{\min }\right)$ is the maximum (minimum) value of variance of the jet constituents' positions with respect to the principal vector. The calculation consists of the following steps:

(1) For each jet the energy-weighted centers in $\eta$ and $\phi$ are calculated as

$$
\bar{\phi}_{\mathrm{jet}}=\frac{\sum_{i} \Delta \phi_{i} E_{i}}{\sum_{i} E_{i}}, \quad \bar{\eta}_{\mathrm{jet}}=\frac{\sum_{i} \Delta \eta_{i} E_{i}}{\sum_{i} E_{i}},
$$

where the energy and position in the $(\eta, \phi)$ plane of the $i$ th constituent with respect to the jet axis are denoted by $E_{i}, \Delta \eta_{i}$ and $\Delta \phi_{i}$.

(2) The PCA is performed to determine the vector $\vec{x}_{1}$ in $(\eta, \phi)$ space that passes through the energyweighted center of the face of the jet and results in a minimum in the variance of the constituents' positions. The angle $\theta$ of this vector with respect to the jet center $\left(\bar{\eta}_{\text {jet }}, \bar{\phi}_{\text {jet }}\right)$ is given by

$$
\tan 2 \theta=\frac{2 \times \sum_{i} E_{i} \Delta \phi_{i} \Delta \eta_{i}}{\sum_{i} E_{i}\left(\Delta \phi_{i}^{2}-\Delta \eta_{i}^{2}\right)}
$$

and the angle of the orthogonal vector $\vec{x}_{2}$ is $\theta-\frac{\pi}{2}$.

(3) The energy-weighted variances $v_{1}$ and $v_{2}$ with respect to $\vec{x}_{1}$ and $\vec{x}_{2}$ are calculated as

$$
\begin{aligned}
& v_{1}=\frac{1}{N} \sum_{i} E_{i}\left(\cos \theta \Delta \eta_{i}-\sin \theta \Delta \phi_{i}\right)^{2}, \\
& v_{2}=\frac{1}{N} \sum_{i} E_{i}\left(\sin \theta \Delta \eta_{i}+\cos \theta \Delta \phi_{i}\right)^{2},
\end{aligned}
$$

where $N$ is the number of constituents.

(4) Finally, the largest value of the variance is assigned to $v_{\max }$ and the smallest to $v_{\min }$. The jet eccentricity ranges from zero for perfectly circular jets to one for jets that appear pencil-like in the $(\eta, \phi)$ plane.

Eccentricity is measured for jets in the mass range $M>$ $100 \mathrm{GeV}$; this is the mass region of interest for the search for a Higgs boson or other massive, hadronically decaying particles predicted in various extensions to the Standard Model.

\section{Planar flow}

A variable complementary to the eccentricity is planar flow $P[10,46,47]$. The planar flow measures the degree to 
which the jet's energy is evenly spread over the plane across the face of the jet (high planar flow) versus spread linearly across the face of the jet (small planar flow).

To calculate planar flow, one first constructs a twodimensional matrix $I_{E}^{k l}$ :

$$
I_{E}^{k l}=\frac{1}{M} \sum_{i} \frac{1}{E_{i}} p_{i, k} p_{i, l} .
$$

Here, $M$ is the jet mass, $E_{i}$ is the energy of the $i$ th constituent of the jet and $p_{i, k}$ and $p_{i, l}$ are the $k$ and $l$ components of its transverse momentum calculated with respect to the jet axis. The planar flow is

$$
P=4 \times \frac{\operatorname{det}\left(I_{E}\right)}{\operatorname{Tr}\left(I_{E}\right)^{2}} .
$$

Vanishing or low planar flow corresponds to a linear energy deposition, as in the case of a two-pronged decay, while completely isotropic energy distributions are characterized by unit planar flow [10]. Jets with many-body kinematics are expected to have a planar flow distribution that peaks towards unity. In general, QCD jets have a rising $P$ distribution that peaks at $P=1$; the hadronization process has contributions from many soft gluons and is largely isotropic. However, jets with high $p_{\mathrm{T}}$ and high mass are well described by a single hard gluon emission. Consequently, these jets have a planar flow distribution that peaks at a low value [13]. The planar flow distributions are measured in the context of boosted, massive particle searches by applying a mass cut, $130<M<210 \mathrm{GeV}$, consistent with the window in which one would expect to observe a boosted top quark decay collimated within a single jet. The contribution from top quark decays in this subset of the data is negligible - here we measure the properties of light-quark and gluon jets that constitute a substantial fraction of the background in boosted top quark measurements.

\section{E. Angularity}

Angularities $\left(\tau_{a}\right)$ are a family of observables that are sensitive to the degree of symmetry in the energy flow inside a jet. The general formula for angularity [10] is given by

$$
\tau_{a}=\frac{1}{M} \sum_{i} E_{i} \sin ^{a} \theta_{i}\left[1-\cos \theta_{i}\right]^{1-a} .
$$

Here $a$ is a parameter that can be chosen to emphasize radiation near the edges $(a<0)$ or core $(a>0)$ of the jet, $M$ is the jet mass, $E_{i}$ is the energy of the $i$ th jet constituent and $\theta_{i}$ is its angle with respect to the jet axis. In the limit of small-angle radiation $\left(\theta_{i} \ll 1\right), \tau_{a}$ is approximated by

$$
\tau_{a} \simeq \frac{2^{(a-1)}}{M} \sum_{i} E_{i} \theta_{i}^{(2-a)} .
$$

Angularities are infrared-safe for $a \leq 2$ [13]. In the analysis presented here, Eq. (9) with a value of $a=-2$ is used. The $\tau_{-2}$ observable can be used as a discriminator for distinguishing QCD jets from boosted particle decays by virtue of the broader tail expected in the QCD distribution [10]. At a given high mass, the angularity of jets with two-body kinematics should peak around a minimum value $\tau_{a}^{\text {peak }} \simeq\left(\frac{M}{2 p_{\mathrm{T}}}\right)^{1-a}$, which corresponds to the two hard constituents being in a symmetric $p_{\mathrm{T}}$ configuration around the jet axis. An estimate for the maximum of the distribution can also be calculated in the limit of small-angle radiation, $\tau_{a}^{\max } \simeq\left(\frac{2}{R}\right)^{a}\left(\frac{M}{2 p_{\mathrm{T}}}\right)$ [13], which corresponds to a hard constituent close to the jet axis and a soft constituent on the jet edge.

The measurement of $\tau_{-2}$ is aimed primarily at testing QCD, which makes predictions for the shape of the $\tau_{-2}$ distribution in jets where the small-angle approximation is valid. For this reason, this measurement is made only for anti- $k_{t}$ jets with $R=0.6$.

Here, $\tau_{-2}$ is measured for jets in the mass range $100<$ $M<130 \mathrm{GeV}$. This mass region is chosen to have minimal contributions from hadronically decaying $W$ or $Z$ bosons or boosted top quarks (PYTHIA predictions estimate a relative fraction below $0.2 \%$ ).

\section{F. Correlations between the observables}

The levels of correlation between the variables presented here provide information that is valuable in deciding which variables may potentially be used together in a search for boosted particles. Here the correlation factors between pairs of variables are calculated as their covariance divided by the product of their standard deviations:

$$
\rho=\frac{\operatorname{cov}(x, y)}{\sigma_{x} \sigma_{y}} .
$$

Summaries of the correlations between all of the observables studied here are shown in Fig. 1 for $R=1.0$ jets in PYTHIA at particle level. The coefficients are shown both with and without a jet mass cut of $M>100 \mathrm{GeV}$ - the individual mass constraints for each observable are dropped here to allow the correlations between them to be calculated. Jets subjected to a mass cut are also restricted to $|\eta|<0.7$. This additional restriction on $\eta$ is applied wherever a mass cut is made on the observables presented here; this has a negligible effect on the shapes of the distributions while allowing direct comparisons with other measurements of the same quantities [13].

The strongest correlations observed are those between jet mass and width (85\%) and between planar flow and eccentricity $(-80 \%)$. The correlation between mass and width reduces considerably when jets are required to be in the kinematic region $M>100 \mathrm{GeV}$. This trend is followed by almost all observables. The planar flow and eccentricity, however, are even more strongly anticorrelated in high-mass jets $(-90 \%)$. The correlation between mass and $p_{\mathrm{T}}$ is weak (12\%-16\%). Angularity is largely uncorrelated with all of the other observables. 

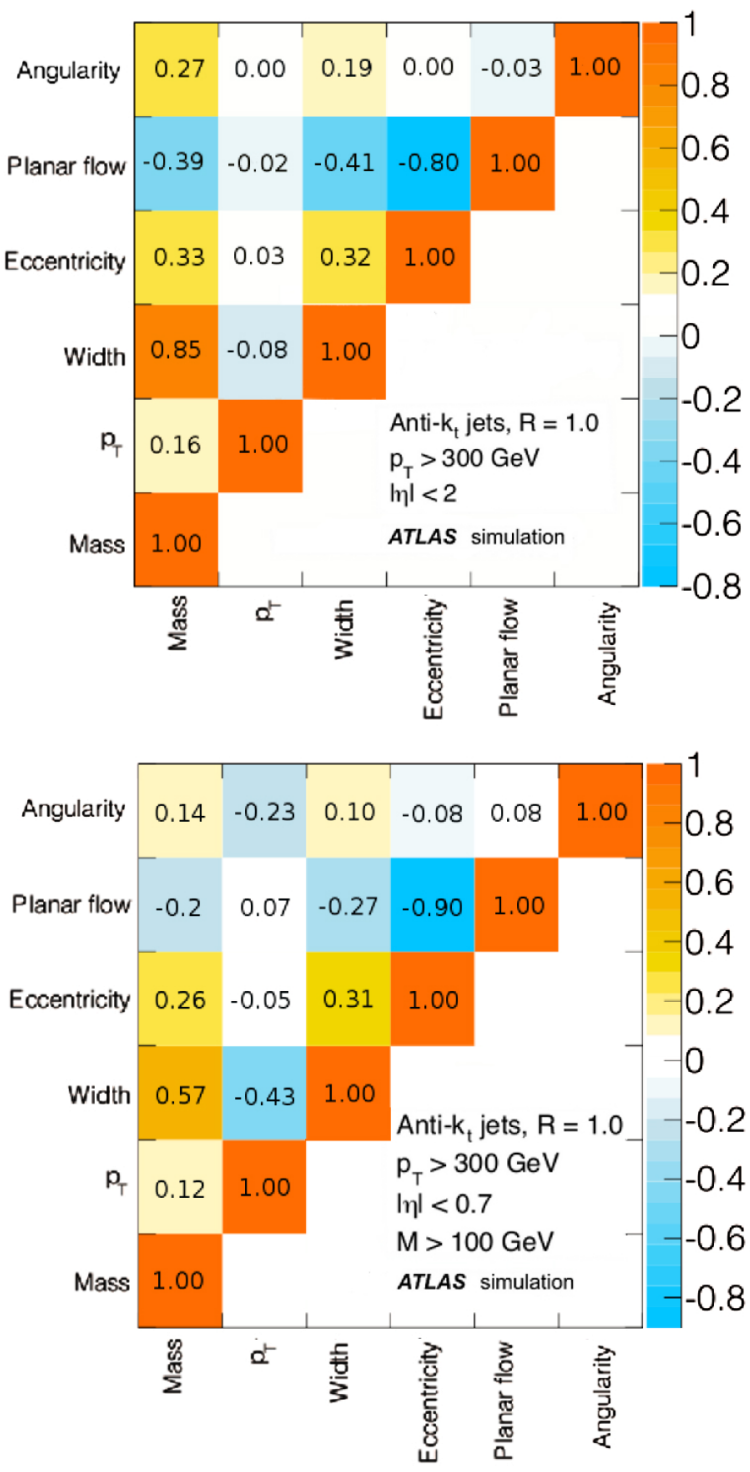

FIG. 1 (color online). The correlation coefficients between pairs of variables calculated in PYTHIA at particle level for $R=1.0$ jets with no mass constraint (top panel) and with a mass constraint of $M>100 \mathrm{GeV}$ (bottom panel).

\section{CORRECTIONS FOR PILEUP AND DETECTOR EFFECTS}

The contribution from pileup is measured using the complementary cone method first introduced by the CDF experiment $[13,48]$. A complementary cone is drawn at a right angle in azimuth to the jet $\left(\phi_{\text {comp }}=\phi_{\text {jet }} \pm \frac{\pi}{2}\right.$, $\left.\eta_{\text {comp }}=\eta_{\text {jet }}\right)$ and the energy deposits in this cone are added into the jet such that the effect on each of the jet properties can be quantified. The shift in each observable after this addition is attributed to pileup and the underlying event (UE), the latter being the diffuse radiation present in all events and partially coherent with the hard scatter. The effects of these two sources are separated by comparing events with $N_{\mathrm{PV}}=1$ (UE only) to those with $N_{\mathrm{PV}}>1$ (UE and pileup): the difference between the average shift for single-vertex and multiple-vertex events is attributed to the contribution from pileup only.

The presence of additional energy in events with $N_{\mathrm{PV}}>1$ affects the substructure observables in different ways; the effect of pileup on the shape of the $\tau_{-2}$ distribution is negligible (below 1\%) in this data set, and so no corrections are applied. The other observables under study have their distributions noticeably distorted by the presence of pileup. The $p_{\mathrm{T}}$-dependent corrections for this effect are applied to the mass, width and eccentricity distributions, while the planar flow distribution of high-mass jets is measured only in events with $N_{\mathrm{PV}}=1$. There are a small number of jets $\left(\sim 100\right.$ anti- $k_{t} R=0.6$ jets $)$ in the highmass range $(M>130 \mathrm{GeV})$, making it too difficult to derive robust pileup corrections for planar flow (which is limited to this mass range in this analysis) in this data set.

\section{A. Pileup corrections for $\boldsymbol{R}=\mathbf{0 . 6}$ jets}

The mass shift due to the UE and pileup in $N_{\mathrm{PV}}=1$ and $N_{\mathrm{PV}}>1$ events is shown in Fig. 2 for $R=0.6$ jets in the range $300<p_{\mathrm{T}}<400 \mathrm{GeV}$. The shift follows the expected behavior, given by

$$
\Delta M=p_{0_{M}}+\frac{p_{1_{M}}}{M}
$$

where $p_{i_{M}}$ and their associated uncertainties are determined from the data. $\Delta M$ is the increase in the jet mass due to the addition of the energy deposits in the complementary cone to the jet. Corrections to the jet mass are limited to the region $M>30 \mathrm{GeV}$, as the $\frac{p_{1_{M}}}{M}$ parametrization uses a leading-order approximation [48] and is only valid for $\Delta M \ll M$. This has a negligible effect on the final measurements in the range $M>20 \mathrm{GeV}$, as illustrated for

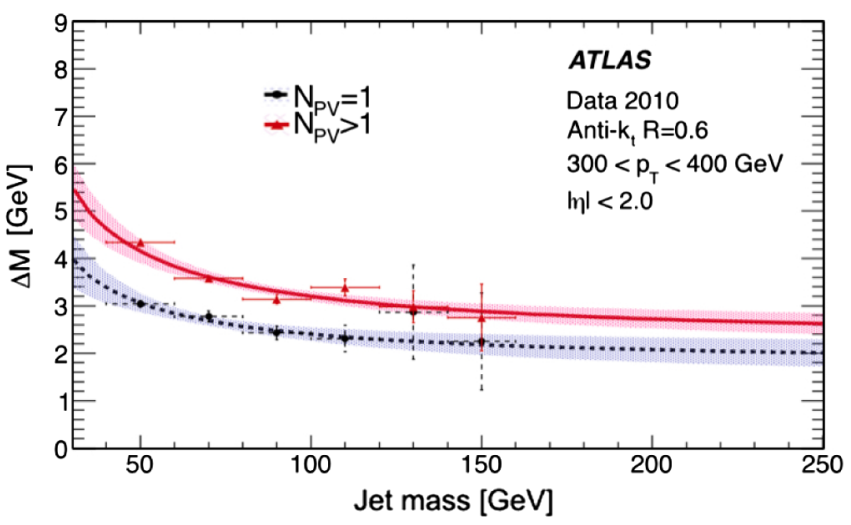

FIG. 2 (color online). The size of the mass shift in anti- $k_{t}$ $R=0.6$ jets with $300<p_{T}<400 \mathrm{GeV}$ in jets with pileup and $\mathrm{UE}\left(N_{\mathrm{PV}}>1\right.$, average $\left.N_{\mathrm{PV}} \simeq 2.2\right)$ and with $\mathrm{UE}$ alone $\left(N_{\mathrm{PV}}=1\right)$. The curves are fits of the form $\Delta M=p_{0_{M}}+\frac{p_{1_{M}}}{M}$. The difference between the curves gives the contribution to the jet mass from pileup only. The $1 \sigma$ uncertainties on the fits are shown in the error bands. 
$R=1.0$ jets in Fig. 3 ; low mass, high- $p_{\mathrm{T}}$ jets tend to have a small contribution from pileup.

The corresponding parametrizations for the shifts in width $\Delta W$ and eccentricity $\Delta \mathcal{E}$ are

$\Delta W=p_{0 W}+p_{1 W} W, \quad \Delta \mathcal{E}=p_{0 \mathcal{E}}+p_{1 \mathcal{E}} \mathcal{E}+p_{2 \mathcal{E}} \mathcal{E}^{2}$.

The pileup corrections for width and eccentricity are applied to jets across the full mass range.
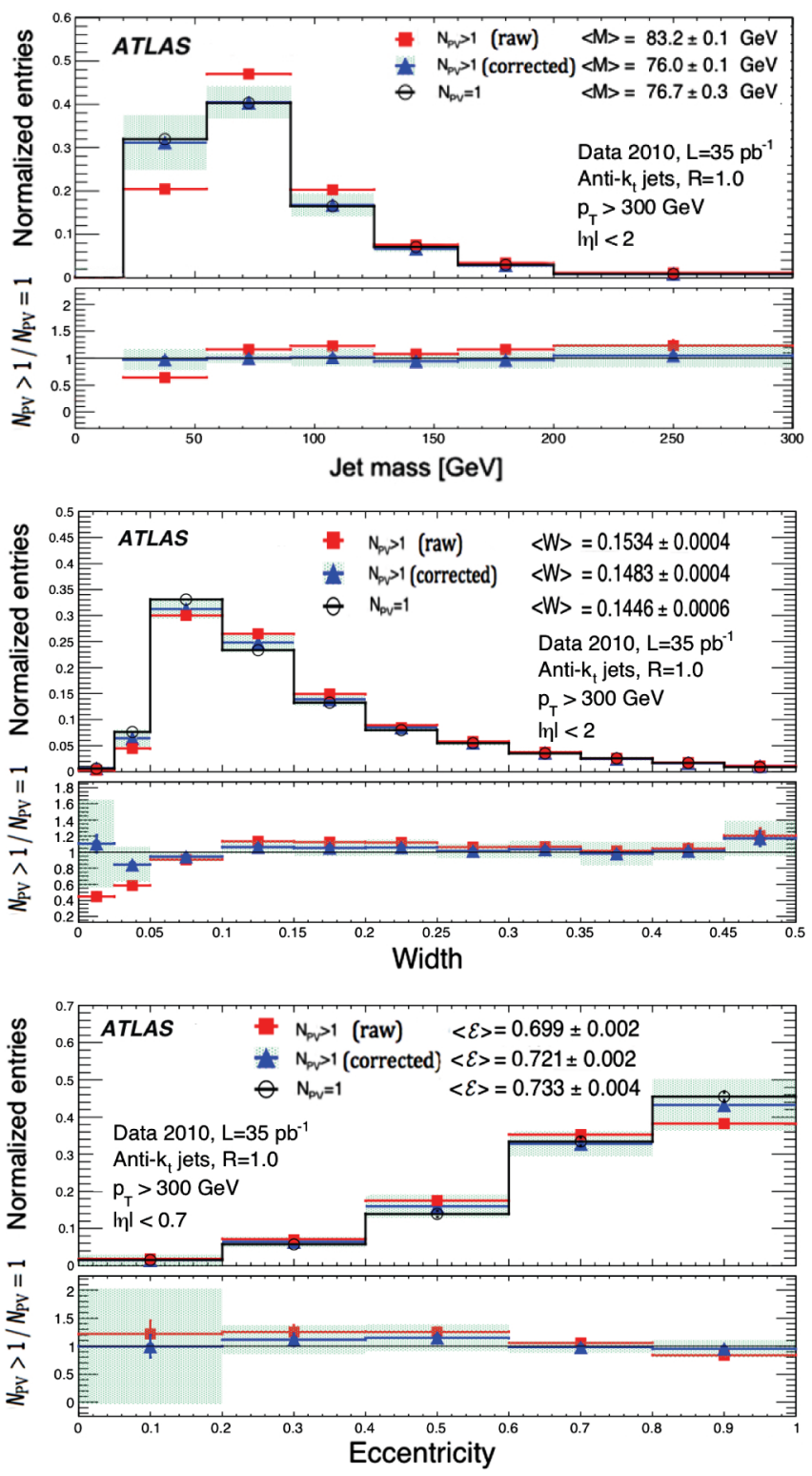

FIG. 3 (color online). The mass, width and eccentricity distributions before and after the pileup corrections. The (red) squares indicate the uncorrected data in the full data set, the (black) circles indicate the subset of this data with $N_{\mathrm{PV}}=1$ and the (blue) triangles indicate the full data set after pileup corrections. The mean value of each distribution is indicated in the legend with the corresponding statistical uncertainty. The lower region of each figure shows the measured ratio of $N_{\mathrm{PV}}>1$ to $N_{\mathrm{PV}}=1$ events.

\section{B. Pileup corrections for $\boldsymbol{R}=\mathbf{1 . 0}$ jets}

The complementary cone technique cannot be applied directly to $R=1.0$ jets due to the high probability of overlap between the complementary cone and the jet; scaling factors are therefore applied to the corrections measured for $R=0.6$ jets.

The scaling behavior is determined experimentally by comparing the pileup dependence in $R=0.6$ and $R=0.4$ jets. For each observable, the shifts for $R=0.4$ jets are fit to a functional form. The shifts for $R=0.6$ jets are then fit to a scaled version of this function, where all parameters are fixed at their $R=0.4$ values and the scaling is the only free parameter of the fit. The measured $R$ dependence is then validated with a comparison between $R=1.0$ jets in $N_{\mathrm{PV}}>1$ and $N_{\mathrm{PV}}=1$ events.

The predicted (observed) behaviors for the scaling of the shifts in mass and width are

$$
\begin{gathered}
\Delta M: p_{i_{M}} \sim R^{4}\left(R^{3.5}\right), \\
\Delta W: p_{0_{W}} \sim R^{3}\left(R^{2.5}\right), \quad p_{1_{W}} \sim R^{2}\left(R^{1}\right) .
\end{gathered}
$$

The phenomenological predictions [49] for scaling are used, and the discrepancies between predictions and observations are considered systematic uncertainties in this procedure.

There is no phenomenological prediction for the scaling of $\Delta \mathcal{E}$ with pileup; therefore the nominal value of the scaling of the shift in this variable is measured in data. The measurements find the scaling of the parametrization to be a function of mass:

$$
\begin{aligned}
& \Delta \mathcal{E}(M<40 \mathrm{GeV}): p_{i_{\mathcal{E}}} \sim R^{2}, \\
& \Delta \mathcal{E}(M \geq 40 \mathrm{GeV}): p_{i_{\mathcal{E}}} \sim R^{3} .
\end{aligned}
$$

The measured scaling is varied between $R^{2}$ and $R^{3}$ across the mass range in order to determine a conservative estimate of the systematic uncertainty introduced by this procedure.

The performance of the pileup correction procedure in the case of mass, width and eccentricity is shown in Fig. 3. The observable most sensitive to pileup is the jet mass; the mean $R=1.0$ jet mass is shifted upwards by $\sim 7 \mathrm{GeV}$ in events with $N_{\mathrm{PV}}>1$, and there is a significant change in the shape of the mass distribution. In the case of jet width and eccentricity, the effect of pileup is a small $(\sim 5 \%)$ shift towards wider, less eccentric jets. This supports the expected behavior: width is less sensitive to pileup than mass, making it a promising alternative to mass as a criterion for selecting jets of interest in boosted particle searches in the high pileup conditions of later LHC operations. For all observables the discrepancies between the pileup-corrected distributions and those for events with $N_{\text {PV }}=1$ are small, and agreement is obtained within the systematic uncertainties on the corrections. 

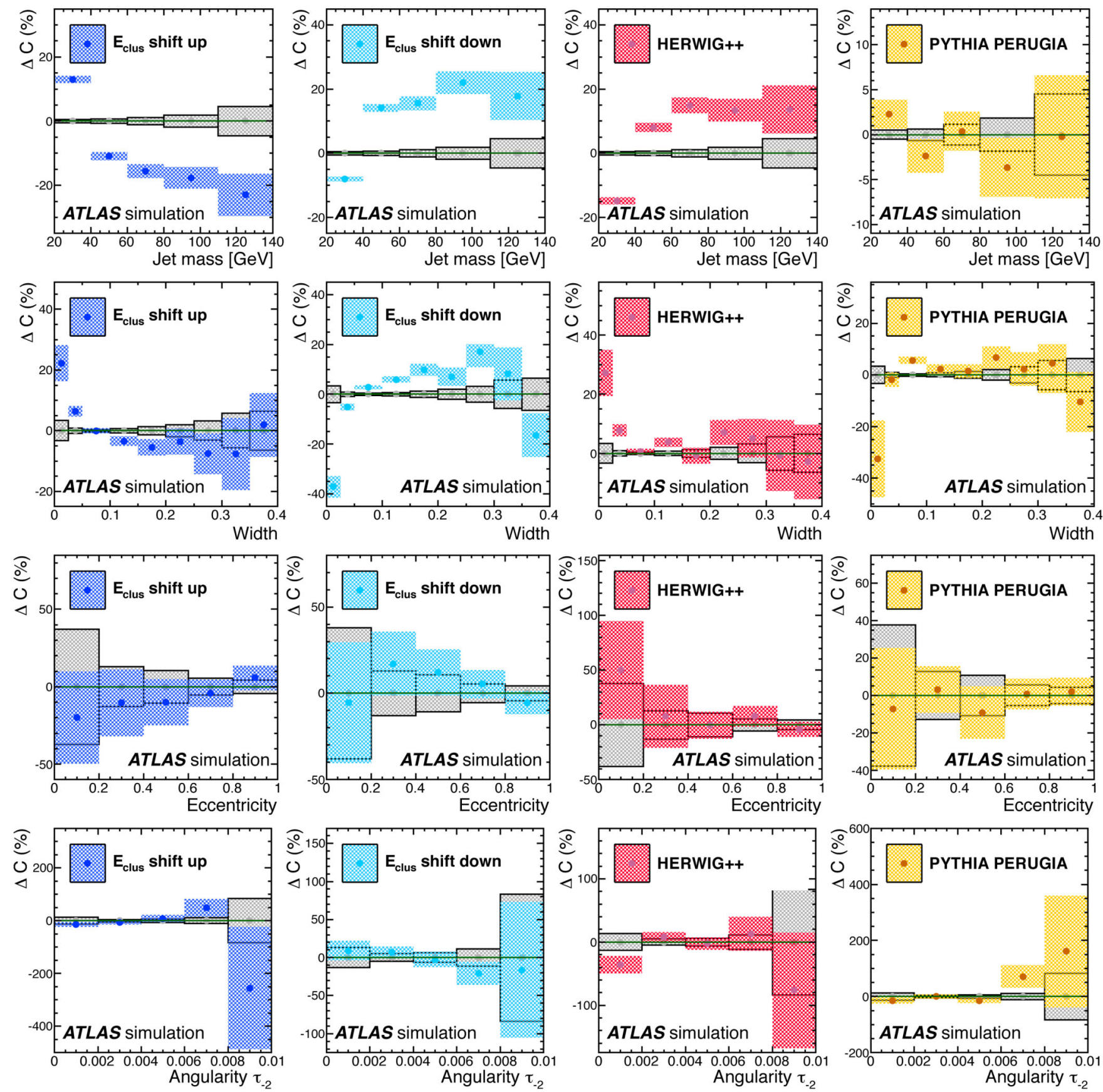

FIG. 4 (color online). The dominant sources of systematic uncertainty on the measurements are those resulting in large variations in the detector correction factors $C$. These correction factors are found bin by bin using $R=0.6$ jets in a PYTHIA AMBT1 sample with upward and downward variations of the cluster energy scale (first and second columns), and by using HERWIG++ (third column) and PYTHIA PERUGIA2010 (fourth column) in place of PYTHIA AMBT1. The differences $\Delta C$ found when comparing the correction factors obtained with the baseline PYTHIA AMBT1 sample are shown here for each of the properties measured in $R=0.6$ jets. The shaded bands indicate the statistical uncertainties.

\section{Corrections for detector effects}

After correcting the distributions for pileup, each distribution is corrected to particle level, using bin-by-bin corrections for detector effects. The bin migrations due to detector effects are determined and controlled by increasing the bin sizes until all bins have a purity and efficiency above 50\% according to Monte Carlo predictions, where purity and efficiency are defined as

$$
p_{i}=\frac{\mathcal{A}_{i}^{\text {part }+ \text { det }}}{\mathcal{A}_{i}^{\text {det }}}, \quad e_{i}=\frac{\mathcal{A}_{i}^{\text {part }+ \text { det }}}{\mathcal{A}_{i}^{\text {part }}} .
$$



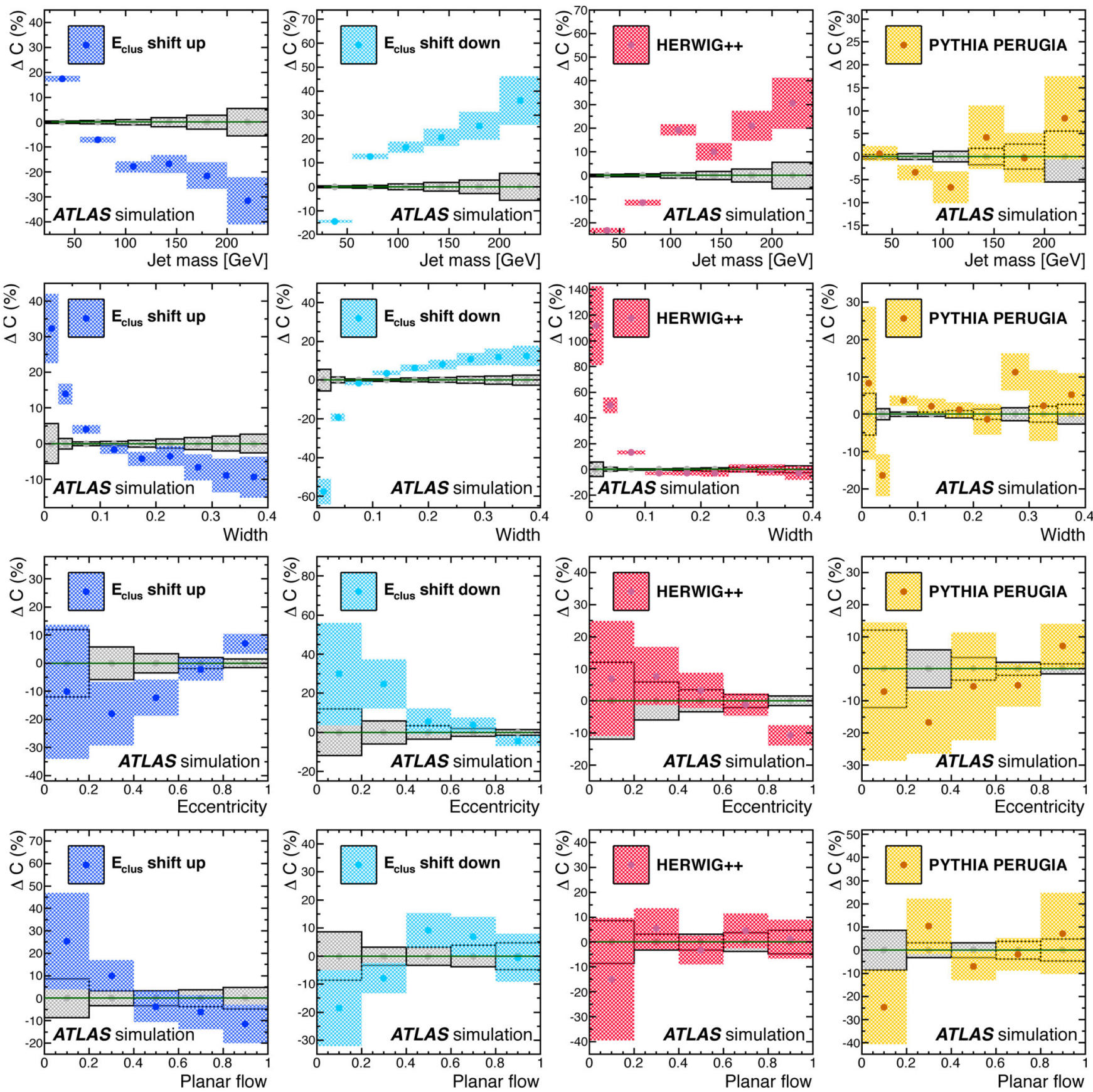

FIG. 5 (color online). The dominant sources of systematic uncertainty on the measurements are those resulting in large variations in the detector correction factors $C$. These correction factors are found bin by bin using $R=1.0$ jets in a PYTHIA AMBT1 sample with upward and downward variations of the cluster energy scale (first and second columns), and by using HERWIG++ (third column) and PYTHIA PERUGIA2010 (fourth column) in place of PYTHIA AMBT1. The differences $\Delta C$ found when comparing the correction factors obtained with the baseline PYTHIA AMBT1 sample are shown here for each of the properties measured in $R=1.0$ jets. The shaded bands indicate the statistical uncertainties.

Here $\mathcal{A}_{i}^{\text {part }+ \text { det }}$ is the number of detector-level jets (reconstructed from locally calibrated clusters) in bin $i$ that have a particle-level jet (reconstructed from stable Monte Carlo particles), matched within $\Delta R<0.2$ and falling in the same bin. $\mathcal{A}_{i}^{\text {part }}$ is the total number of particle-level jets in bin $i$ and $\mathcal{A}_{i}^{\text {det }}$ is the total number of detector-level jets in bin $i$.

The particle-level value for an observable in bin $i$ is found by multiplying its measured value by the relevant correction factor $C_{i}$ : 

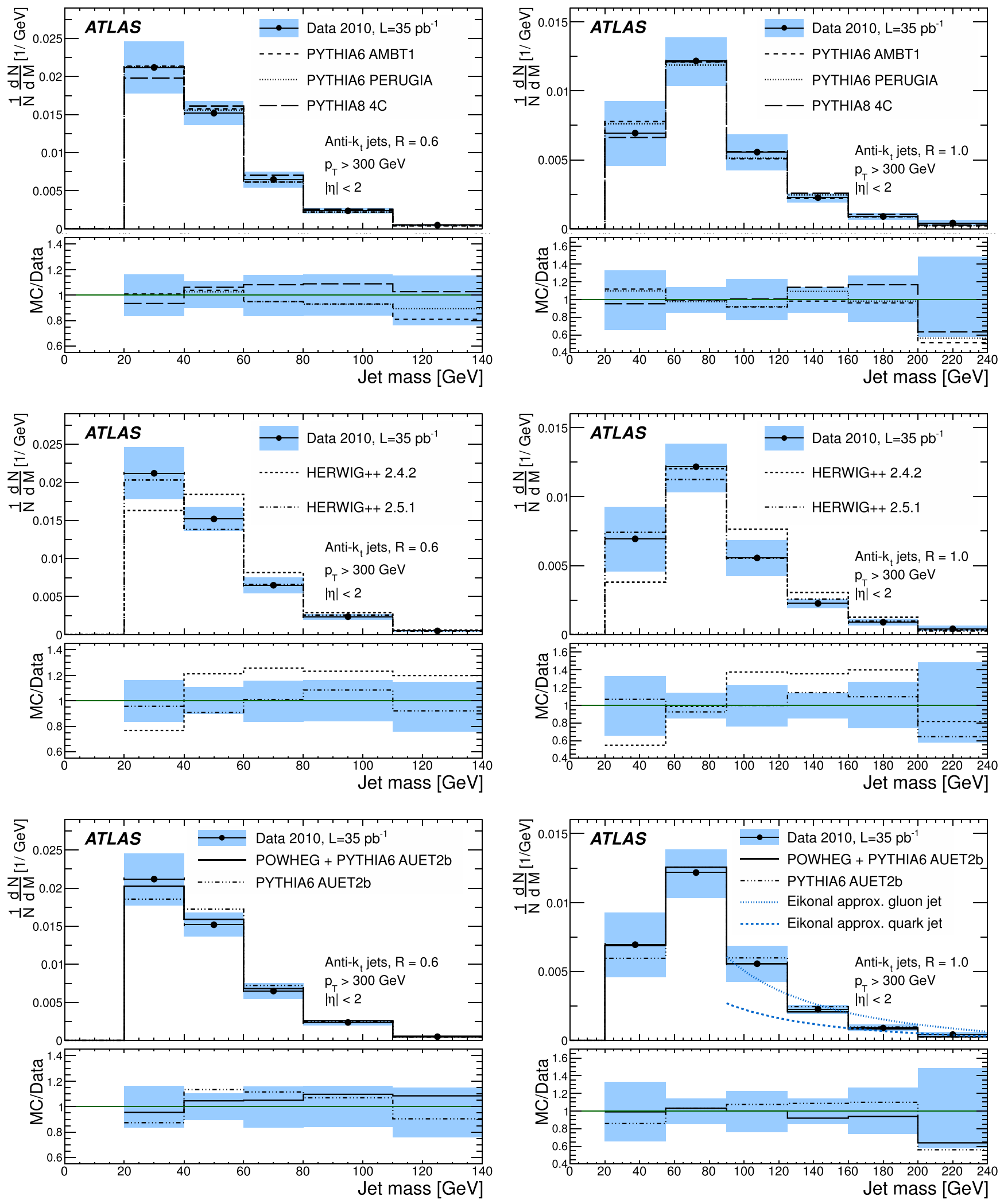

FIG. 6 (color online). The jet mass distributions for leading $p_{\mathrm{T}}$, anti- $k_{t} R=0.6$ (left) and $R=1.0$ (right) jets in the full 2010 data set, corrected for pileup and corrected to particle level. The data are compared to various tunes of PYTHIA 6 and PYTHIA 8 (top), HERWIG ++2.4 .2 and 2.5.1 (center) and PYTHIA AUET2B with and without POWHEG (bottom). The eikonal approximation of NLO QCD for quark and gluon jets is also included for the $R=1.0$ case (right, bottom). The shaded bands indicate the sum of statistical and systematic uncertainties. 


$$
C_{i}=\frac{\mathcal{A}_{i}^{\text {part }}}{\mathcal{A}_{i}^{\text {det }}}
$$

The size of the corrections varies quite significantly between observables and between bins, being around $20 \%$ for mass (5\%-10\% around the peak, $20 \%$ elsewhere) and width $(30 \%$ in the peak for $R=0.6$ jets, $1 \%-5 \%$ elsewhere). The corrections for eccentricity are below $10 \%$ in the peak, increasing to $40 \%$ in the most sparsely populated bin. The detector corrections for angularity and planar flow are smaller, generally around $0 \%-5 \%$.

\section{SYSTEMATIC UNCERTAINTIES}

The experimental systematic uncertainties can be divided into three categories: how well modeled the observables are in Monte Carlo simulations (Sec. VII A), the modeling of the detector material and cluster reconstruction (Sec. VII B) and the pileup corrections (Sec. VII C). These are evaluated by determining the difference in the factors obtained after the application of systematic variations to the samples used in the correction for detector effects. The dominant sources of uncertainty, described in detail below, arise from varying the cluster energy scale (CES) and from the differences found when the calculation of detector corrections is done using the HERWIG++ Monte Carlo sample in place of PYTHIA AMBT1. These dominant effects are shown in Fig. 4 for $R=0.6$ jets and Fig. 5 for $R=1.0$ jets.

\section{A. Uncertainties on the Monte Carlo model}

The distributions are corrected to particle level using the correction factors $C_{i}$ determined with a specific Monte Carlo generator, inclusive of parton shower, hadronization and UE model, which in this case is PYTHIA with the AMBT1 tune. To determine the uncertainty introduced on the final measurement by choosing this particular model to calculate the detector correction factors, the differences in these $C_{i}$ are found when the PYTHIA AMBT1 tune is replaced with the PERUGIA2010 tune, and with HERWIG++ (2.4.2).

A primary source of the uncertainty on the mass measurements is due to the observed differences in the detector correction factors between HERWIG++ and PYTHIA, with uncertainties ranging between $10 \%$ and $20 \%$ as shown in Figs. 4 and 5.

\section{B. Uncertainties on the detector material description and cluster reconstruction}

Performance studies [50] have shown that there is excellent agreement between the measured positions of clusters and tracks in data, indicating no systematic misalignment between the calorimeter and inner detector. The Monte Carlo modeling of the position of clusters with respect to tracks is also good, indicating that the
TABLE I. Measured values of the anti- $k_{t} R=0.6$ jet mass distribution given with their statistical and systematic uncertainties.

\begin{tabular}{lc}
\hline \hline $\operatorname{Bin}(\mathrm{GeV})$ & $\frac{1}{N} \frac{d N}{d M} \pm \operatorname{stat} \pm \operatorname{sys}\left(\times 10^{-4}\right)\left[\frac{1}{\mathrm{GeV}}\right]$ \\
\hline $20-40$ & $212 \pm 2 \pm 34$ \\
$40-60$ & $152 \pm 1 \pm 16$ \\
$60-80$ & $65 \pm 1_{-11}^{+10}$ \\
$80-110$ & $24 \pm 1 \pm 4$ \\
$110-140$ & $5.0 \pm 0.2_{-1.2}^{+0.8}$ \\
\hline \hline
\end{tabular}

detector simulation models the calorimeter position resolution adequately; however, there remains a small discrepancy between data and Monte Carlo in the mean and RMS of the track-cluster separation. This source of uncertainty is taken into account by (Gaussian) smearing the positions of simulated clusters in $\eta$ and $\phi$ by $5 \mathrm{mrad}$. This smearing is done independently in $\eta$ and $\phi$, and the impact on the measurement of the correction factors for each observable, bin by bin, is quantified by taking the difference, $\Delta C_{i}$, between the correction factors obtained before and after the position smearing. Smearing the positions in $\eta$ and $\phi$ results in small $\Delta C_{i}$ for mass and shapes alike, introducing uncertainties that do not exceed $5 \%$ in any bin.

The variation on the CES follows the procedure used by previous studies [3] according to

$$
p_{\text {clus,new }}=p_{\text {clus }} \times\left(1 \pm 0.05 \times\left(1+\frac{1.5}{p_{\mathrm{T}} / \mathrm{GeV}}\right)\right),
$$

where $p_{\text {clus }}$ is each component of the cluster's fourmomentum and $p_{\mathrm{T}}$ is the cluster $p_{\mathrm{T}}$ in GeV. The CES is varied up and down independently for each momentum component of each cluster, and the correction factors are recalculated in each case as before. The CES is a large source of systematic uncertainty in the measurement of mass (of order $20 \%$ across the mass range) and width (of order $10 \%$ beyond the first two bins). The effects of varying the CES are, in general, smaller for the eccentricity, planar flow and angularity measurements, all

TABLE II. Measured values of the anti- $k_{t} R=1.0$ jet mass distribution given with their statistical and systematic uncertainties.

\begin{tabular}{lc}
\hline \hline Bin $(\mathrm{GeV})$ & $\frac{1}{N} \frac{d N}{d M} \pm \operatorname{stat} \pm \operatorname{sys}\left(\times 10^{-4}\right)\left[\frac{1}{\mathrm{GeV}}\right]$ \\
\hline $20-55$ & $69 \pm 1_{-24}^{+23}$ \\
$55-90$ & $122 \pm 1_{-18}^{+17}$ \\
$90-125$ & $56 \pm 1 \pm 13$ \\
$125-160$ & $22.6 \pm 0.4_{-3.4}^{+3.2}$ \\
$160-200$ & $9.0 \pm 0.2_{-2.3}^{+2.4}$ \\
$200-240$ & $4.3 \pm 0.2_{-1.8}^{+2.1}$ \\
\hline \hline
\end{tabular}



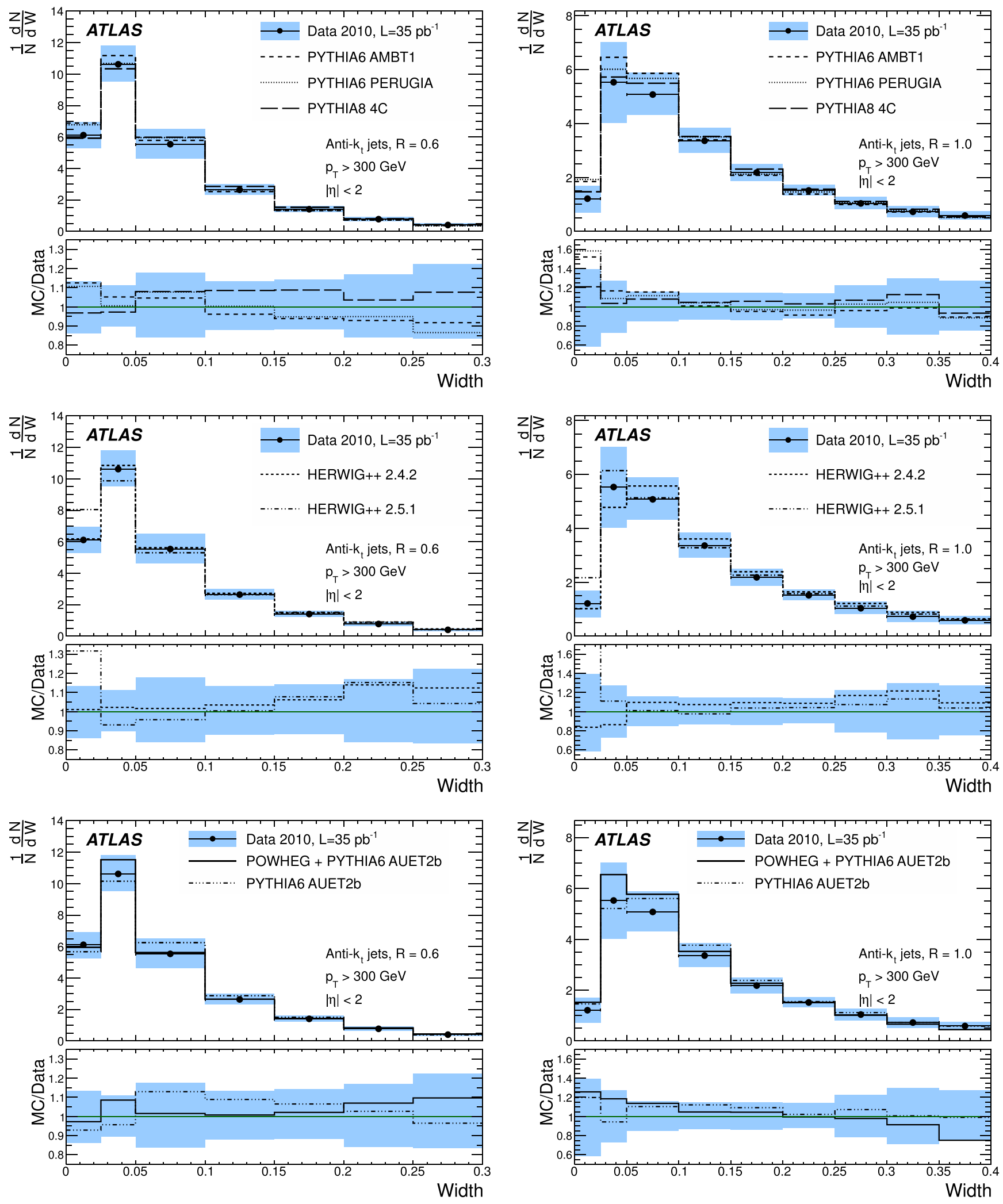

FIG. 7 (color online). The jet width distributions for leading $p_{\mathrm{T}}$, anti- $k_{t} R=0.6$ (left) and $R=1.0$ (right) jets in the full 2010 data set, corrected for pileup and corrected to particle level. 
of which are made on high-mass jets only. The effects of varying the CES on all observables are shown with the label $E_{\text {clus }}$ in Fig. 4 for $R=0.6$ jets and Fig. 5 for $R=1.0$ jets.

The uncertainty introduced as a result of losing energy due to dead material in the detector is taken into account by discarding a fraction of low energy $(E<2.5 \mathrm{GeV})$ clusters, following the technique and utilizing the observations of a previous study of the single hadron response at $\sqrt{s}=$ $900 \mathrm{GeV}$ [51]. Clusters are not included in jet reconstruction if they satisfy

$$
r \leq \mathcal{P}\left(E_{0}\right) \times e^{-2 E},
$$

where $r$ is a random number $r \in(0,1], \mathcal{P}\left(E_{0}\right)$ is the measured uncertainty $(28 \%)$ on the probability that a particle does not leave a cluster in the calorimeter, and $E$ is the cluster energy in $\mathrm{GeV}$. The impact on the measurement of each observable is quantified by comparing the correction factors before and after this dropping of low energy clusters. The impact of this variation is small, resulting in a contribution to the systematic uncertainty of less than a few percent in all measurements.

\section{Uncertainties on the pileup corrections}

There is a statistical uncertainty on the fit $f\left(x, p_{\mathrm{T}}, M\right)$ describing the pileup correction $\Delta x$ for observable $x$ in $R=0.6$ jets. Dedicated studies have shown that the parametrizations of the pileup corrections in data and in PYTHIA AMBT1 Monte Carlo with simulated pileup agree, within the statistical uncertainties, for jets across the $p_{\mathrm{T}}$ range considered. The statistical uncertainties on these fits are accounted for by implementing $+1 \sigma$ and $-1 \sigma$ variations independently, as shown for mass in Fig. 2. The correction factors are recalculated, and in each case the difference is taken as a contribution to the systematic uncertainty on the measurement. This is a small contribution to the overall systematic uncertainty on the measurements, contributing at most a few percent in bins that are statistically limited, and is a negligible $(<1 \%)$ effect elsewhere.

TABLE III. Measured values of the anti- $k_{t} R=0.6$ jet width distribution given with their statistical and systematic uncertainties.

\begin{tabular}{lc}
\hline \hline Bin & $\frac{1}{N} \frac{d N}{d W} \pm$ stat $\pm \operatorname{sys}\left(\times 10^{-1}\right)$ \\
\hline $0-0.025$ & $61.1 \pm 1.2_{-8.5}^{+8.2}$ \\
$0.025-0.05$ & $106 \pm 1_{-11}^{+12}$ \\
$0.05-0.1$ & $55.3 \pm 0.4_{-9}^{+10}$ \\
$0.1-0.15$ & $26.4 \pm 0.3_{-3}^{+4}$ \\
$0.15-0.2$ & $14.0 \pm 0.3 \pm 2$ \\
$0.2-0.25$ & $7.7 \pm 0.2_{-1.2}^{+1.3}$ \\
$0.25-0.3$ & $4.0 \pm 0.2_{-0.7}^{+0.9}$ \\
\hline \hline
\end{tabular}

For $R=1.0$ jets, the correction factors are scaled using the phenomenological predictions described in Sec. VIB. These scaling factors are also calculated in data and in PYTHIA AMBT1 Monte Carlo with simulated pileup; good agreement is observed, indicating that the effect of pileup on jets is well modeled. In the case of mass and width, where there is a phenomenological prediction for the scaling, this prediction is used for the determination of the nominal scaling factors and the variation is taken from the scaling factors found in data. In the case of eccentricity there is no phenomenological prediction for the scaling of the pileup corrections with $R$, so the behavior observed in data is used. The $R$ scaling of the pileup corrections for eccentricity is dependent on jet mass, so the variations found in data across the mass range are taken as the systematic variations.

The uncertainties introduced by the pileup corrections contribute a small amount (in general $1 \%-2 \%$ ) to the total systematic uncertainties on the mass, width and eccentricity.

The sources of systematic uncertainty described above are added in quadrature with the statistical uncertainty in each bin and symmetrized where appropriate (the contributions from the cluster energy scale and parametrization of the pileup corrections are determined separately for upward and downward fluctuations, and so are not symmetrized).

\section{RESULTS}

The distributions of jet characteristics presented in this section are corrected for detector effects and are compared to Monte Carlo predictions at the particle level. In the case of mass and $\tau_{-2}$, comparison is also made between data and the eikonal approximation [46] of NLO QCD. The results shown here are available in HEPDATA [52,53] and the analysis and data are available as a RIVET [54,55] routine.

TABLE IV. Measured values of the anti- $k_{t} R=1.0$ jet width distribution given with their statistical and systematic uncertainties.

\begin{tabular}{lc}
\hline \hline Bin & $\frac{1}{N} \frac{d N}{d W} \pm$ stat $\pm \operatorname{sys}\left(\times 10^{-1}\right)$ \\
\hline $0-0.025$ & $12.1 \pm 0.5_{-5.0}^{+4.8}$ \\
$0.025-0.05$ & $55.3 \pm 0.8 \pm 15.0$ \\
$0.05-0.1$ & $50.8 \pm 0.4_{-7.5}^{+8.2}$ \\
$0.1-0.15$ & $33.6 \pm 0.3_{-4.5}^{+4.9}$ \\
$0.15-0.2$ & $21.8 \pm 0.3_{-3.0}^{+3.3}$ \\
$0.2-0.25$ & $15.1 \pm 0.2_{-1.9}^{+2.1}$ \\
$0.25-0.3$ & $10.4 \pm 0.2_{-2.2}^{+2.4}$ \\
$0.3-0.35$ & $7.3 \pm 0.2_{-2.1}^{+2.2}$ \\
$0.35-0.4$ & $5.9 \pm 0.2_{-1.4}^{+1.6}$ \\
\hline \hline
\end{tabular}



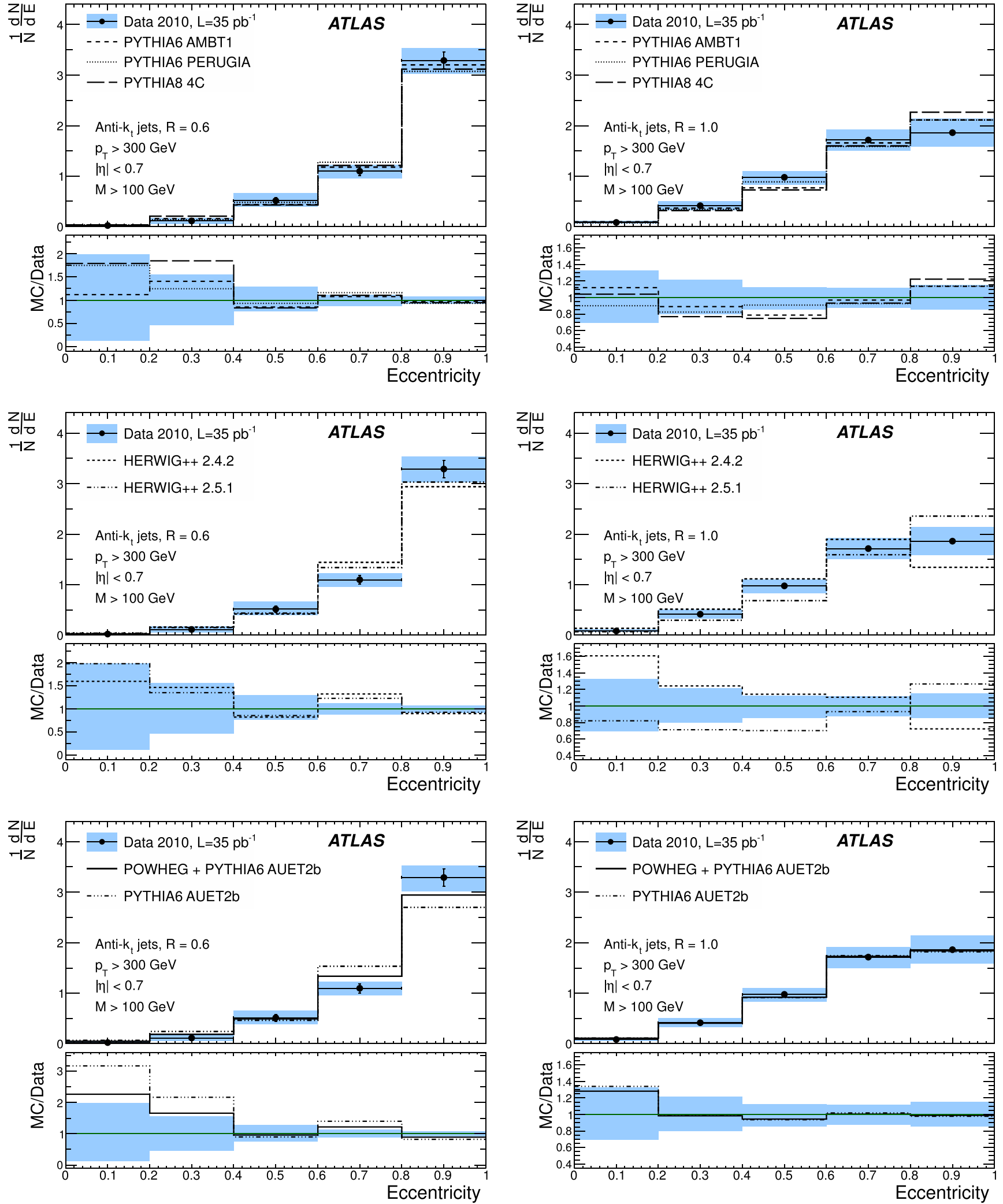

FIG. 8 (color online). The jet eccentricity distributions for high-mass $(M>100 \mathrm{GeV})$, leading $p_{\mathrm{T}}$, anti- $k_{t} R=0.6$ (left) and $R=$ 1.0 (right) jets in the full 2010 data set, corrected for pileup and corrected to particle level. 
G. AAD et al.

TABLE V. Measured values of the eccentricity distribution for anti- $k_{t} R=0.6$ jets with $M>100 \mathrm{GeV}$, given with their statistical and systematic uncertainties.

\begin{tabular}{lc}
\hline \hline Bin & $\frac{1}{N} \frac{d N}{d \mathcal{E}} \pm \operatorname{stat} \pm \operatorname{sys}\left(\times 10^{-1}\right)$ \\
\hline $0-0.2$ & $0.2 \pm 0.1 \pm 0.2$ \\
$0.2-0.4$ & $1.1 \pm 0.3 \pm 0.6$ \\
$0.4-0.6$ & $5.1 \pm 0.7_{-1.3}^{+1.5}$ \\
$0.6-0.8$ & $11.0 \pm 0.9_{-1.4}^{+1.3}$ \\
$0.8-1.0$ & $32.9 \pm 1.7_{-2.7}^{+2.4}$ \\
\hline \hline
\end{tabular}

\section{A. Jet mass}

The jet mass distributions are shown in Fig. 6 for jets satisfying $p_{\mathrm{T}}>300 \mathrm{GeV}$ and $|\eta|<2$, corrected to the particle level, and the corresponding numerical values are given in Tables I and II.

In the case of $R=1.0$ jets, the data are compared to the calculations for jet masses derived at NLO QCD in the eikonal approximation:

$$
J \simeq \alpha_{\mathrm{S}} \frac{4 C_{\mathrm{c}}}{\pi M} \log \left(\frac{1}{z} \tan \left(\frac{R}{2}\right) \sqrt{4-z^{2}}\right),
$$

where $J$ is the value of the jet mass distribution at $M, \alpha_{\mathrm{S}}$ is the strong coupling constant, $z=M / p_{\mathrm{T}}$, c represents the flavor of the parton which initiated the jet and $C_{\mathrm{c}}=\frac{4}{3}$ (3) for quarks (gluons). The strong coupling constant is calculated using the PYTHIA prediction of the average jet $p_{\mathrm{T}} \simeq$ $365 \mathrm{GeV}$ and has the value of $\alpha_{\mathrm{S}}=0.0994$. Theoretical uncertainties for such predictions are sizable (more than $30 \%$ ) [46] in the region above the mass peak. The lower mass region $M \lesssim 90 \mathrm{GeV}$ is strongly affected by nonperturbative physics and as such cannot be predicted by such calculations. The size and shape of the high-mass tail is in rough agreement with the analytical eikonal approximation for NLO QCD for jet masses above $90 \mathrm{GeV}$, with most of the data points lying between the predictions for quarkinitiated and gluon-initiated jets. QCD LO calculations predict that the jets in this sample should be roughly $50 \%$ quark initiated, with this fraction increasing as a function of the jet $p_{\mathrm{T}}$ cut [56].

Also included in Fig. 6 are a number of PYTHIA, HERWIG++, and POWHEG predictions for the jet mass distributions. Unlike the analytical calculation discussed

TABLE VI. Measured values of the eccentricity distribution for anti- $k_{t} R=1.0$ jets with $M>100 \mathrm{GeV}$, given with their statistical and systematic uncertainties.

\begin{tabular}{lc}
\hline \hline Bin & $\frac{1}{N} \frac{d N}{d \mathcal{E}} \pm$ stat $\pm \operatorname{sys}\left(\times 10^{-1}\right)$ \\
\hline $0-0.2$ & $0.8 \pm 0.1 \pm 0.3$ \\
$0.2-0.4$ & $4.2 \pm 0.2 \pm 0.9$ \\
$0.4-0.6$ & $9.8 \pm 0.3_{-1.4}^{+1.2}$ \\
$0.6-0.8$ & $17.2 \pm 0.4_{-2.2}^{+2.0}$ \\
$0.8-1.0$ & $18.6 \pm 0.4_{-2.7}^{+2.8}$ \\
\hline \hline
\end{tabular}

PHYSICAL REVIEW D 86, 072006 (2012)
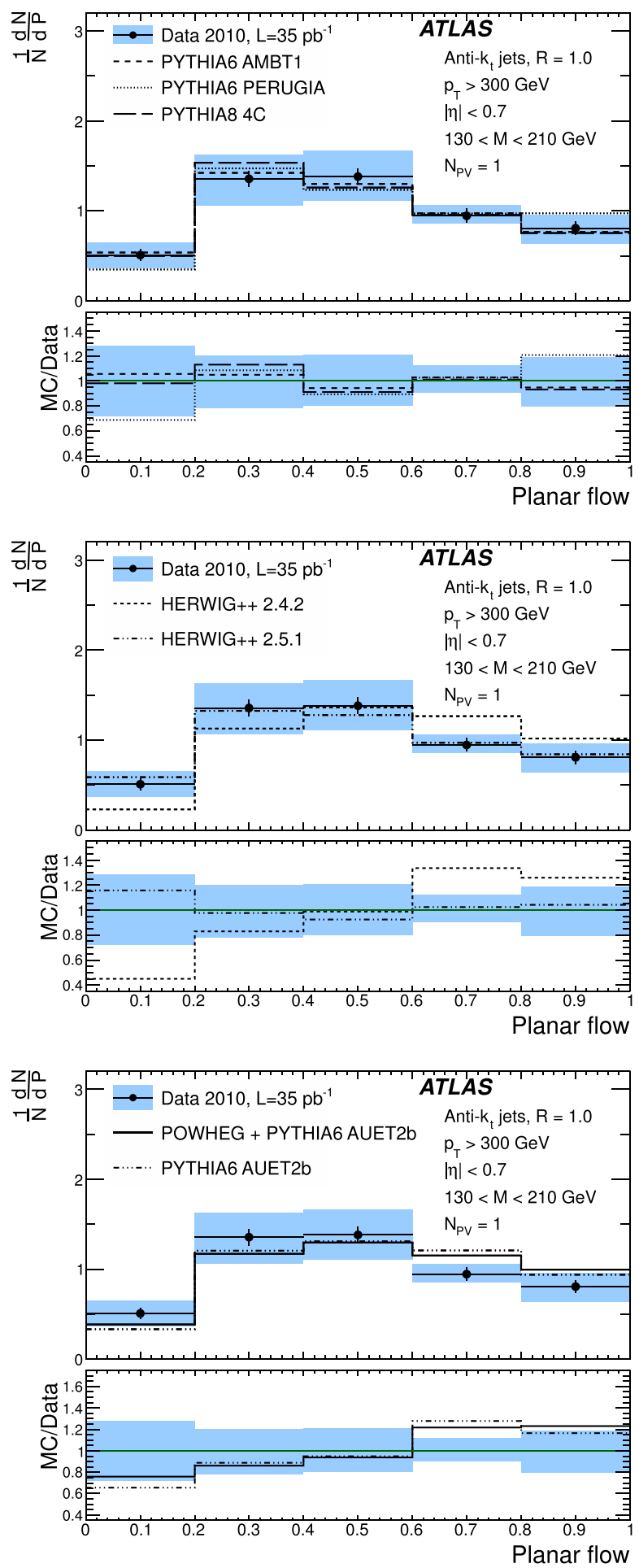

FIG. 9 (color online). The jet planar flow distributions for high-mass $(130<M<210 \mathrm{GeV})$, leading $p_{\mathrm{T}}$, anti- $k_{t} R=1.0$ jets in $N_{\mathrm{PV}}=1$ events, corrected to particle level.

above, the Monte Carlo predictions are meaningful down to the low mass region due to the inclusion of soft radiation and hadronization. The PYTHIA calculation describes the data well. The HERWIG++ 2.4 .2 prediction indicates a significant shift to a higher jet mass that is inconsistent with 
the data and the other Monte Carlo predictions, while the more recent HERWIG++ 2.5.1 generator is in much better agreement with the data. POWHEG+PYTHIA is in good agreement with data within systematic uncertainties across the whole mass range.

\section{B. Width}

The jet width distributions are shown in Fig. 7 for anti- $k_{t}$ jets reconstructed with distance parameters of $R=0.6$ and 1.0 , and the corresponding numerical values are given in Tables III and IV. There is significant variation between the different Monte Carlo predictions in the first bin, beyond which there is good agreement between the distribution measured in data and all the predictions.

\section{Eccentricity}

The eccentricity distributions for high-mass $(M>$ $100 \mathrm{GeV}$ ) anti- $k_{t}$ jets reconstructed with distance parameters of $R=0.6$ and $R=1.0$ are shown in Fig. 8, and the corresponding numerical values are given in Tables $\mathrm{V}$ and VI. The Monte Carlo predictions generally describe the data, while some small discrepancies can be observed between the various predictions and between predictions and data.

\section{Planar flow}

The planar flow distributions are shown only for events known to be uncontaminated by pileup, corresponding to events with $N_{\mathrm{PV}}=1$. These distributions are shown in Fig. 9 for jets reconstructed with the anti- $k_{t}$ algorithm with $R=1.0$ for the mass range $130<M<210 \mathrm{GeV}$, and the corresponding numerical values are given in Table VII. The HERWIG++ 2.4.2 generator predicts jets with a more planar, isotropic energy distribution than is observed in data, while version 2.5 .1 provides a very accurate description of the planar flow. The various PYTHIA and POWHEG Monte Carlo predictions also describe the data well, within uncertainties.

\section{E. Angularity}

The $\tau_{-2}$ distribution for anti- $k_{t} R=0.6$ jets in the mass region $100<M<130 \mathrm{GeV}$ is presented in Fig. 10, and

TABLE VII. Measured values of the planar flow distribution for anti- $k_{t} R=1.0$ jets in $N_{\mathrm{PV}}=1$ events with $130<M<210 \mathrm{GeV}$, given with their statistical and systematic uncertainties.

\begin{tabular}{lc}
\hline \hline Bin & $\frac{1}{N} \frac{d N}{d P} \pm$ stat $\pm \operatorname{sys}\left(\times 10^{-1}\right)$ \\
\hline $0-0.2$ & $5.1 \pm 0.7 \pm 1.4$ \\
$0.2-0.4$ & $13.6 \pm 0.9_{-3.0}^{+2.7}$ \\
$0.4-0.6$ & $13.8 \pm 0.9_{-2.7}^{+2.9}$ \\
$0.6-0.8$ & $9.5 \pm 0.8_{-0.9}^{+1.2}$ \\
$0.8-1.0$ & $8.1 \pm 0.7_{-1.7}^{+1.5}$ \\
\hline \hline
\end{tabular}
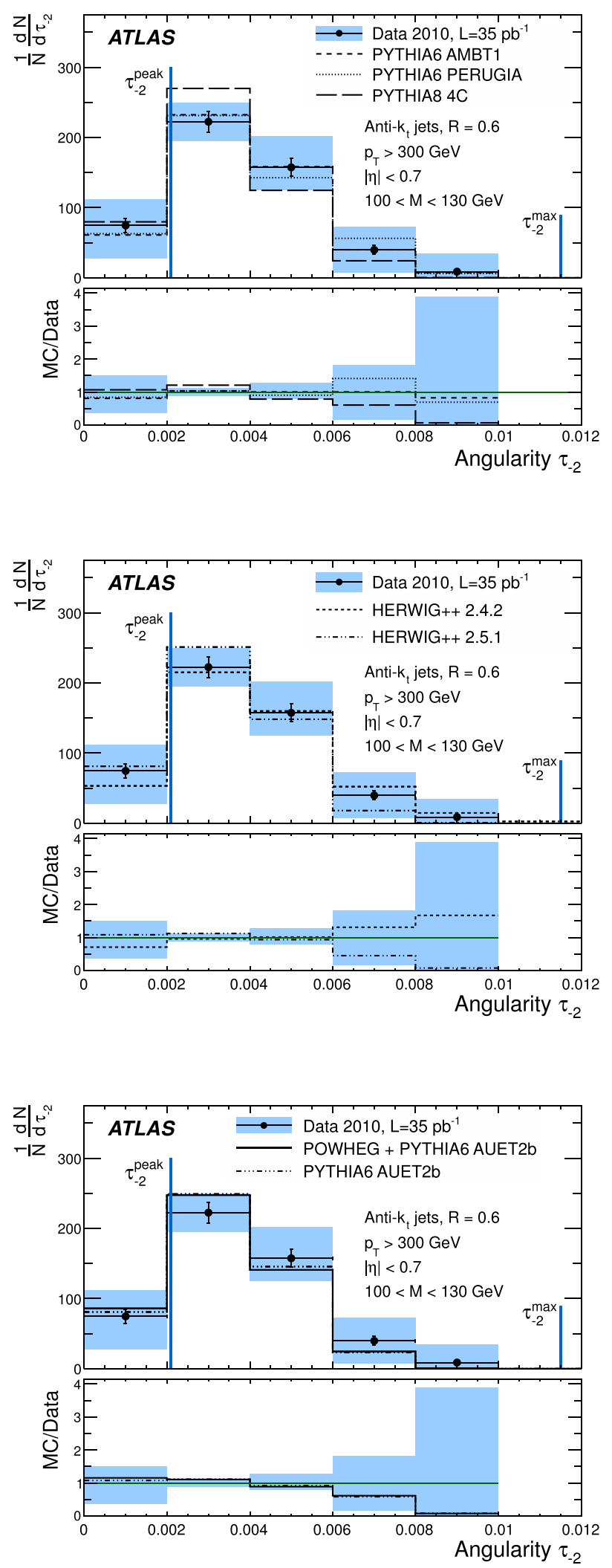

FIG. 10 (color online). The angularity $\tau_{-2}$ distributions for leading $p_{\mathrm{T}}$, anti- $k_{t} R=0.6$ jets in the mass range $100<M<$ $130 \mathrm{GeV}$, in the full 2010 data set, corrected to particle level. The peak and maximum positions predicted by the small-angle approximation of Eq. (10) are indicated. 
TABLE VIII. Measured values of the angularity $\tau_{-2}$ distribution for anti- $k_{t} R=0.6$ jets with $100<M<130 \mathrm{GeV}$, given with their statistical and systematic uncertainties.

\begin{tabular}{lc}
\hline \hline Bin & $\frac{1}{N} \frac{d N}{d \tau_{-2}} \pm$ stat \pm sys \\
\hline $0-0.002$ & $75 \pm 10_{-46}^{+38}$ \\
$0.002-0.004$ & $223 \pm 15_{-28}^{+27}$ \\
$0.004-0.006$ & $158 \pm 13_{-33}^{+44}$ \\
$0.006-0.008$ & $40 \pm 6 \pm 33$ \\
$0.008-0.010$ & $9 \pm 5_{-9}^{+26}$ \\
\hline \hline
\end{tabular}

the corresponding numerical values are given in Table VIII. The QCD predictions for the peak position and the maximum value of $\tau_{-2}$ [13], calculated using the averages $\langle M\rangle=111 \mathrm{GeV}$ and $\left\langle p_{\mathrm{T}}\right\rangle=434 \mathrm{GeV}$ of the jets in this kinematic region, are also shown on the distributions. Good agreement is observed between the data and the Monte Carlo simulation for the shape of the $\tau_{-2}$ distribution.

The comparison between data and the analytic QCD prediction is limited by the intrinsic resolution of the data distribution; however, there is good agreement between theory and data within these limitations. The position of the peak of the distribution, $\tau_{-2}^{\text {peak }}$, indicates that the majority of jets in this data set can be described by a twobody substructure in a symmetric $p_{\mathrm{T}}$ configuration with respect to the jet axis. No jets are observed above the smallangle kinematic limit, $\tau_{-2}^{\max }$.

\section{CONCLUSIONS}

The properties of high- $p_{\mathrm{T}}(>300 \mathrm{GeV})$ jets reconstructed with the anti- $k_{t}$ jet algorithm have been studied in $p p$ collisions at a center-of-mass energy of $7 \mathrm{TeV}$. There is good agreement between data and PYTHIA for all observables, and the POWHEG+PYTHIA prediction describes the mass distribution well for jets with $M>20 \mathrm{GeV}$. HERWIG++ 2.4.2 predicts jets with a slightly more isotropic energy flow and higher mass than observed in data, while HERWIG++ 2.5.1 predictions are in good agreement with the data. The angularity measurement of high-mass jets agrees with the small-angle QCD approximations.

\section{ACKNOWLEDGMENTS}

We thank CERN for the very successful operation of the LHC, as well as the support staff from our institutions without whom ATLAS could not be operated efficiently. We acknowledge the support of ANPCyT, Argentina; YerPhI, Armenia; ARC, Australia; BMWF, Austria; ANAS, Azerbaijan; SSTC, Belarus; CNPq and FAPESP, Brazil; NSERC, NRC and CFI, Canada; CERN; CONICYT, Chile; CAS, MOST and NSFC, China; COLCIENCIAS, Colombia; MSMT CR, MPO CR and VSC CR, Czech Republic; DNRF, DNSRC and Lundbeck Foundation, Denmark; EPLANET and ERC, European Union; IN2P3-CNRS, CEA-DSM/IRFU, France; GNAS, Georgia; BMBF, DFG, HGF, MPG and AvH Foundation, Germany; GSRT, Greece; ISF, MINERVA, GIF, DIP and Benoziyo Center, Israel; INFN, Italy; MEXT and JSPS, Japan; CNRST, Morocco; FOM and NWO, Netherlands; RCN, Norway; MNiSW, Poland; GRICES and FCT, Portugal; MERYS (MECTS), Romania; MES of Russia and ROSATOM, Russian Federation; JINR; MSTD, Serbia; MSSR, Slovakia; ARRS and MVZT, Slovenia; DST/NRF, South Africa; MICINN, Spain; SRC and Wallenberg Foundation, Sweden; SER, SNSF and Cantons of Bern and Geneva, Switzerland; NSC, Taiwan; TAEK, Turkey; STFC, the Royal Society and Leverhulme Trust, United Kingdom; DOE and NSF, United States of America. The crucial computing support from all WLCG partners is acknowledged gratefully, in particular from CERN and the ATLAS Tier-1 facilities at TRIUMF (Canada), NDGF (Denmark, Norway, Sweden), CC-IN2P3 (France), KIT/GridKA (Germany), INFNCNAF (Italy), NL-T1 (Netherlands), PIC (Spain), ASGC (Taiwan), RAL (UK) and BNL (USA) and in the Tier-2 facilities worldwide.
[1] ATLAS Collaboration, Phys. Rev. D 86, 014022 (2012).

[2] CMS Collaboration, Phys. Lett. B 700, 187 (2011).

[3] ATLAS Collaboration, Phys. Rev. D 83, 052003 (2011).

[4] CMS Collaboration, J. High Energy Phys. 06 (2012) 160.

[5] ATLAS Collaboration, J. High Energy Phys. 05 (2012) 128.

[6] ATLAS Collaboration, New J. Phys. 13, 053044 (2011).

[7] CMS Collaboration, Phys. Rev. Lett. 106, 201804 (2011).

[8] A. Altheimer et al., J. Phys. G 39, 063001 (2012).
[9] J. Butterworth, A. R. Davison, M. Rubin, and G. P. Salam, Phys. Rev. Lett. 100, 242001 (2008).

[10] L. Almeida, S. Lee, G. Perez, G. Sterman, I. Sung, and J. Virzi, Phys. Rev. D 79, 074017 (2009).

[11] Y. Cui, Z. Han, and M. Schwartz, Phys. Rev. D 83, 074023 (2011).

[12] S. Chekanov and J. Proudfoot, Phys. Rev. D 81, 114038 2010.

[13] T. Aaltonen et al. (CDF Collaboration), Phys. Rev. D 85, 091101 (2012). 
[14] T. Plehn, G. Salam, and M. Spannowsky, Phys. Rev. Lett. 104, 111801 (2010).

[15] ATLAS Collaboration, New J. Phys. 13, 053033 (2011).

[16] ATLAS Collaboration, Report No. ATLAS-CONF-2010069, 2010 (https://cdsweb.cern.ch/record/1281344).

[17] ATLAS Collaboration, JINST 3, S08003 (2008).

[18] T. Sjostrand, S. Mrenna, and P. Skands, J. High Energy Phys. 05 (2006) 026.

[19] ATLAS Collaboration, Report No. ATLAS-CONF-2010031, 2010 (https://cdsweb.cern.ch/record/1277665).

[20] A. Sherstnev and R. Thorne, Eur. Phys. J. C 55, 553 (2008).

[21] B. Andersson, G. Gustafson, G. Ingelman, and T. Sjöstrand, Phys. Rep. 97, 31 (1983).

[22] B. Andersson, G. Gustafson, and G. Soderberg, Z. Phys. C 20, 317 (1983).

[23] M. Bowler, Z. Phys. C 11, 169 (1981).

[24] ATLAS Collaboration, Phys. Lett. B 688, 21 (2010).

[25] ATLAS Collaboration, Report No. ATLAS-CONF-2010024, 2010 (https://cdsweb.cern.ch/record/1277656).

[26] P. Skands, Phys. Rev. D 82, 074018 (2010).

[27] ATLAS Collaboration, Phys. Rev. D 84, 054001 (2011).

[28] H. L. Lai, J. Huston, S. Kuhlmann, J. Morfin, F. Olness, J. F. Owens, J. Pumplin, and W. K. Tung, Eur. Phys. J. C 12, 375 (2000).

[29] M. Bahr et al., Eur. Phys. J. C 58, 639 (2008).

[30] ATLAS Collaboration, Report No. ATL-PHYS-PUB2011-009, 2011 (https://cdsweb.cern.ch/record/1363300).

[31] ATLAS Collaboration, Report No. ATL-PHYS-PUB2011-014, 2011 (https://cdsweb.cern.ch/record/1400677).

[32] T. Sjostrand, S. Mrenna, and P. Skands, Comput. Phys. Commun. 178, 852 (2008).

[33] S. Alioli, K. Hamilton, P. Nason, C. Oleari, and E. Re, J. High Energy Phys. 04 (2011) 081.

[34] P. Nason, J. High Energy Phys. 11 (2004) 040.

[35] S. Frixione, P. Nason, and C. Oleari, J. High Energy Phys. 11 (2007) 070.
[36] S. Alioli, P. Nason, C. Oleari, and E. Re, J. High Energy Phys. 06 (2010) 043.

[37] ATLAS Collaboration, Eur. Phys. J. C 70, 823 (2010).

[38] S. Agostinelli et al., Nucl. Instrum. Methods Phys. Res., Sect. A 506, 250 (2003).

[39] G. Folger and J. Wellisch, arXiv:nucl-th/0306007.

[40] H. W. Bertini, Phys. Rev. 188, 1711 (1969).

[41] ATLAS Collaboration, Eur. Phys. J. C 71, 1630 (2011).

[42] ATLAS Collaboration, Report No. ATLAS-CONF-2011011, 2011 (https://cdsweb.cern.ch/record/1334563).

[43] ATLAS Collaboration, Report No. ATL-LARG- PUB2008-002, 2008 (https://cdsweb.cern.ch/record/1099735).

[44] M. Cacciari, G. Salam, and G. Soyez, J. High Energy Phys. 04 (2008) 063.

[45] ATLAS Collaboration, arXiv:1112.6426 [Eur. Phys. J. C (to be published)].

[46] L. Almeida, S. Lee, G. Perez, I. Sung, and J. Virzi, Phys. Rev. D 79, 074012 (2009).

[47] J. Thaler and L.-T. Wang, J. High Energy Phys. 7 (2008) 092.

[48] R. Alon, E. Duchovni, G. Perez, A. Pranko, and P. Sinervo, Phys. Rev. D 84, 114025 (2011).

[49] M. Dasgupta, L. Magnea, and G. Salam, J. High Energy Phys. 02 (2008) 055.

[50] ATLAS Collaboration, Phys. Rev. Lett. 106, 172002, 2011.

[51] ATLAS Collaboration, arXiv:1203.1302 [Eur. Phys. J. C (to be published)].

[52] A. Buckley and M. Whalley, Proc. Sci., ACAT2010 (2010) 067 [http://arxiv.org/abs/1006.0517v2].

[53] HEPDATA: ATLAS measurements of the properties of jets for boosted particle searches, http://hepdata.cedar.ac.uk/ view/red4992.

[54] A. Buckley et al., arXiv:1003.0694v6.

[55] RIVET routine: ATLAS measurements of the properties of jets for boosted particle searches, http://rivet.hepforge.org/ analyses.

[56] J. Gallichio and M. Schwartz, J. High Energy Phys. 10 (2011) 103.

G. Aad, ${ }^{47}$ B. Abbott, ${ }^{110}$ J. Abdallah, ${ }^{11}$ S. Abdel Khalek, ${ }^{114}$ A. A. Abdelalim,${ }^{48}$ O. Abdinov, ${ }^{10}$ B. Abi,,${ }^{11}$ M. Abolins,${ }^{87}$ O. S. AbouZeid,${ }^{157}$ H. Abramowicz,${ }^{152}$ H. Abreu, ${ }^{135}$ E. Acerbi ${ }^{88 a}, 88 b$ B. S. Acharya, ${ }^{163 a, 163 b}$ L. Adamczyk, ${ }^{37}$ D. L. Adams, ${ }^{24}$ T. N. Addy,${ }^{55}$ J. Adelman, ${ }^{175}$ S. Adomeit, ${ }^{97}$ P. Adragna, ${ }^{74}$ T. Adye, ${ }^{128}$ S. Aefsky, ${ }^{22}$ J. A. Aguilar-Saavedra, ${ }^{123 b, b}$ M. Aharrouche ${ }^{80}$ S. P. Ahlen,${ }^{21}$ F. Ahles,${ }^{47}$ A. Ahmad, ${ }^{147}$ M. Ahsan, ${ }^{40}$ G. Aielli, ${ }^{132 a, 132 b}$ T. Akdogan, ${ }^{18 a}$ T. P. A. Akesson, ${ }^{78}$ G. Akimoto, ${ }^{154}$ A. V. Akimov, ${ }^{93}$ A. Akiyama, ${ }^{65}$ M. S. Alam, ${ }^{1}$ M. A. Alam, ${ }^{75}$ J. Albert, ${ }^{168}$ S. Albrand,${ }^{54}$ M. Aleksa, ${ }^{29}$ I. N. Aleksandrov, ${ }^{63}$ F. Alessandria ${ }^{88 a}$ C. Alexa, ${ }^{25 a}$ G. Alexander, ${ }^{152}$ G. Alexandre, ${ }^{48}$ T. Alexopoulos, ${ }^{9}$ M. Alhroob, ${ }^{163 a, 163 c}$ M. Aliev, ${ }^{15}$ G. Alimonti, ${ }^{88 a}$ J. Alison, ${ }^{119}$ B. M. M. Allbrooke, ${ }^{17}$ P. P. Allport,${ }^{72}$ S. E. Allwood-Spiers, ${ }^{52}$ J. Almond,${ }^{81}$ A. Aloisio, ${ }^{101 a, 101 b}$ R. Alon, ${ }^{171}$ A. Alonso, ${ }^{78}$ B. Alvarez Gonzalez, ${ }^{87}$ M. G. Alviggi, ${ }^{101 \mathrm{a}, 101 \mathrm{~b}}$ K. Amako, ${ }^{64}$ C. Amelung, ${ }^{22}$ V. V. Ammosov, ${ }^{127, a}$ A. Amorim, ${ }^{123 a, c}$ N. Amram, ${ }^{152}$ C. Anastopoulos ${ }^{29}$ L. S. Ancu,${ }^{16}$ N. Andari, ${ }^{114}$ T. Andeen, ${ }^{34}$ C. F. Anders,${ }^{57 b}$ G. Anders, ${ }^{57 a}$ K. J. Anderson, ${ }^{30}$ A. Andreazza, ${ }^{88 a, 88 b}$ V. Andrei ${ }^{57 a}$ X. S. Anduaga, ${ }^{69}$ P. Anger, ${ }^{43}$ A. Angerami ${ }^{34}$ F. Anghinolfi, ${ }^{29}$ A. Anisenkov, ${ }^{106}$ N. Anjos, ${ }^{123 a}$ A. Annovi, ${ }^{46}$ A. Antonaki, ${ }^{8}$ M. Antonelli, ${ }^{46}$ A. Antonov, ${ }^{95}$ J. Antos, ${ }^{143 b}$ F. Anulli, ${ }^{131 a}$ S. Aoun, ${ }^{82}$ L. Aperio Bella, ${ }^{4}$ R. Apolle,${ }^{117, d}$ G. Arabidze, ${ }^{87}$ I. Aracena, ${ }^{142}$ Y. Arai, ${ }^{64}$ A. T. H. Arce, ${ }^{44}$ S. Arfaoui, ${ }^{147}$ J-F. Arguin, ${ }^{14}$ E. Arik, ${ }^{18 a, a}$ M. Arik, ${ }^{18 a}$ A. J. Armbruster, ${ }^{86}$ O. Arnaez ${ }^{80}$ V. Arnal, ${ }^{79}$ C. Arnault, ${ }^{114}$ A. Artamonov, ${ }^{94}$ G. Artoni, ${ }^{131 \mathrm{a}, 131 \mathrm{~b}}$ D. Arutinov,${ }^{20}$ S. Asai, ${ }^{154}$ R. Asfandiyarov,${ }^{172}$ S. Ask,${ }^{27}$ B. Asman, ${ }^{145 a, 145 b}$ L. Asquith, ${ }^{5}$ K. Assamagan, ${ }^{24}$ A. Astbury, ${ }^{168}$ B. Aubert, ${ }^{4}$ E. Auge, ${ }^{114}$ K. Augsten, ${ }^{126}$ M. Aurousseau, ${ }^{144 a}$ G. Avolio, ${ }^{162}$ R. Avramidou, ${ }^{9}$ D. Axen, ${ }^{167}$ G. Azuelos, ${ }^{92, \mathrm{e}}$ Y. Azuma, ${ }^{154}$ M. A. Baak, ${ }^{29}$ G. Baccaglioni, ${ }^{88 \mathrm{a}}$ C. Bacci, ${ }^{133 a, 133 b}$ A. M. Bach,${ }^{14}$ H. Bachacou, ${ }^{135} \mathrm{~K}$. Bachas,${ }^{29}$ M. Backes,${ }^{48}$ M. Backhaus ${ }^{20}$ 
E. Badescu, ${ }^{25 a}$ P. Bagnaia, ${ }^{131 a, 131 b}$ S. Bahinipati, ${ }^{2}$ Y. Bai, ${ }^{32 a}$ D. C. Bailey, ${ }^{157}$ T. Bain, ${ }^{157}$ J. T. Baines, ${ }^{128}$ O. K. Baker ${ }^{175}$ M. D. Baker ${ }^{24}$ S. Baker,${ }^{76}$ E. Banas, ${ }^{38}$ P. Banerjee, ${ }^{92}$ Sw. Banerjee, ${ }^{172}$ D. Banfi, ${ }^{29}$ A. Bangert, ${ }^{149}$ V. Bansal, ${ }^{168}$ H. S. Bansil, ${ }^{17}$ L. Barak, ${ }^{171}$ S. P. Baranov, ${ }^{93}$ A. Barbaro Galtieri, ${ }^{14}$ T. Barber,${ }^{47}$ E. L. Barberio, ${ }^{85}$ D. Barberis, ${ }^{49 a, 49 b}$ M. Barbero, ${ }^{20}$ D. Y. Bardin, ${ }^{63}$ T. Barillari, ${ }^{98}$ M. Barisonzi, ${ }^{174}$ T. Barklow, ${ }^{142}$ N. Barlow,${ }^{27}$ B. M. Barnett, ${ }^{128}$ R. M. Barnett, ${ }^{14}$ A. Baroncelli, ${ }^{133 a}$ G. Barone, ${ }^{48}$ A. J. Barr, ${ }^{117}$ F. Barreiro, ${ }^{79}$

J. Barreiro Guimarães da Costa, ${ }^{56}$ P. Barrillon, ${ }^{114}$ R. Bartoldus, ${ }^{142}$ A. E. Barton, ${ }^{70}$ V. Bartsch, ${ }^{148}$ R. L. Bates, ${ }^{52}$ L. Batkova, ${ }^{143}$ J. R. Batley, ${ }^{27}$ A. Battaglia, ${ }^{16}$ M. Battistin, ${ }^{29}$ F. Bauer, ${ }^{135}$ H. S. Bawa, ${ }^{142, f}$ S. Beale, ${ }^{97}$ T. Beau, ${ }^{77}$ P. H. Beauchemin,${ }^{160}$ R. Beccherle, ${ }^{49 a}$ P. Bechtle,${ }^{20}$ H. P. Beck, ${ }^{16}$ A. K. Becker,${ }^{174}$ S. Becker, ${ }^{97}$ M. Beckingham, ${ }^{137}$ K. H. Becks, ${ }^{174}$ A. J. Beddall, ${ }^{18 \mathrm{c}}$ A. Beddall, ${ }^{18 \mathrm{c}}$ S. Bedikian, ${ }^{175}$ V. A. Bednyakov, ${ }^{63}$ C. P. Bee,${ }^{82}$ M. Begel,${ }^{24}$ S. Behar Harpaz, ${ }^{151}$ M. Beimforde, ${ }^{98}$ C. Belanger-Champagne, ${ }^{84}$ P. J. Bell, ${ }^{48}$ W. H. Bell, ${ }^{48}$ G. Bella, ${ }^{152}$ L. Bellagamba, ${ }^{19 a}$ F. Bellina,${ }^{29}$ M. Bellomo, ${ }^{29}$ A. Belloni, ${ }^{56}$ O. Beloborodova, ${ }^{106, g}$ K. Belotskiy, ${ }^{95}$ O. Beltramello, ${ }^{29}$ O. Benary, ${ }^{152}$ D. Benchekroun, ${ }^{134 a}$ K. Bendtz, ${ }^{145 a, 145 b}$ N. Benekos, ${ }^{164}$ Y. Benhammou, ${ }^{152}$ E. Benhar Noccioli ${ }^{48}$ J. A. Benitez Garcia, ${ }^{158 b}$ D. P. Benjamin, ${ }^{44}$ M. Benoit, ${ }^{114}$ J. R. Bensinger, ${ }^{22}$ K. Benslama, ${ }^{129}$ S. Bentvelsen, ${ }^{104}$ D. Berge, ${ }^{29}$ E. Bergeaas Kuutmann, ${ }^{41}$ N. Berger ${ }^{4}$ F. Berghaus, ${ }^{168}$ E. Berglund, ${ }^{104}$ J. Beringer, ${ }^{14}$ P. Bernat, ${ }^{76}$ R. Bernhard, ${ }^{47}$ C. Bernius, ${ }^{24}$ T. Berry,${ }^{75}$ C. Bertella, ${ }^{82}$ A. Bertin,,${ }^{19 a, 19 b}$ F. Bertolucci, ${ }^{121 a, 121 b}$ M. I. Besana, ${ }^{88 a, 88 b}$ G. J. Besjes, ${ }^{103}$ N. Besson, ${ }^{135}$ S. Bethke, ${ }^{98}$ W. Bhimji, ${ }^{45}$ R. M. Bianchi, ${ }^{29}$ M. Bianco,${ }^{71 a, 71 b}$ O. Biebel, ${ }^{97}$ S. P. Bieniek, ${ }^{76}$ K. Bierwagen, ${ }^{53}$ J. Biesiada, ${ }^{14}$ M. Biglietti, ${ }^{133 a}$ H. Bilokon, ${ }^{46}$ M. Bindi, ${ }^{19 a, 19 b}$ S. Binet,${ }^{114}$ A. Bingul, ${ }^{18 \mathrm{c}}$ C. Bini, ${ }^{131 \mathrm{a}, 131 \mathrm{~b}}$ C. Biscarat, ${ }^{177}$ U. Bitenc, ${ }^{47}$ K. M. Black,${ }^{21}$ R. E. Blair, ${ }^{5}$ J.-B. Blanchard, ${ }^{135}$ G. Blanchot, ${ }^{29}$ T. Blazek, ${ }^{143 a}$ C. Blocker, ${ }^{22}$ J. Blocki, ${ }^{38}$ A. Blondel,,${ }^{48}$ W. Blum,${ }^{80}$ U. Blumenschein, ${ }^{53}$ G. J. Bobbink, ${ }^{104}$ V. B. Bobrovnikov, ${ }^{106}$ S. S. Bocchetta, ${ }^{78}$ A. Bocci, ${ }^{44}$ C. R. Boddy, ${ }^{117}$ M. Boehler, ${ }^{41}$ J. Boek, ${ }^{174}$ N. Boelaert, ${ }^{35}$ J. A. Bogaerts, ${ }^{29}$ A. Bogdanchikov, ${ }^{106}$ A. Bogouch, ${ }^{89, a}$ C. Bohm, ${ }^{145 a}$ J. Bohm, ${ }^{124}$ V. Boisvert ${ }^{75}$

T. Bold, ${ }^{37}$ V. Boldea, ${ }^{25 a}$ N. M. Bolnet, ${ }^{135}$ M. Bomben, ${ }^{77}$ M. Bona ${ }^{74}$ M. Bondioli, ${ }^{162}$ M. Boonekamp, ${ }^{135}$ C. N. Booth, ${ }^{138}$ S. Bordoni, ${ }^{77}$ C. Borer, ${ }^{16}$ A. Borisov, ${ }^{127}$ G. Borissov, ${ }^{70}$ I. Borjanovic, ${ }^{12 a}$ M. Borri, ${ }^{81}$ S. Borroni, ${ }^{86}$ V. Bortolotto, ${ }^{133 a, 133 \mathrm{~b}}$ K. Bos, ${ }^{104} \mathrm{D}$. Boscherini, ${ }^{19 \mathrm{a}} \mathrm{M}$. Bosman, ${ }^{11} \mathrm{H}$. Boterenbrood, ${ }^{104} \mathrm{D}$. Botterill, ${ }^{128} \mathrm{~J}$. Bouchami, ${ }^{92}$ J. Boudreau, ${ }^{122}$ E. V. Bouhova-Thacker,${ }^{70}$ D. Boumediene, ${ }^{33}$ C. Bourdarios,,${ }^{114}$ N. Bousson, ${ }^{82}$ A. Boveia,${ }^{30}$ J. Boyd,${ }^{29}$ I. R. Boyko, ${ }^{63}$ I. Bozovic-Jelisavcic, ${ }^{12 b}$ J. Bracinik, ${ }^{17}$ P. Branchini, ${ }^{133 a}$ A. Brandt, ${ }^{7}$ G. Brandt,${ }^{117}$ O. Brandt,${ }^{53}$

U. Bratzler, ${ }^{155}$ B. Brau, ${ }^{83}$ J. E. Brau, ${ }^{113}$ H. M. Braun, ${ }^{174, a}$ S. F. Brazzale, ${ }^{163 a, 163 c}$ B. Brelier, ${ }^{157}$ J. Bremer, ${ }^{29}$ K. Brendlinger, ${ }^{119}$ R. Brenner, ${ }^{165}$ S. Bressler, ${ }^{171}$ D. Britton, ${ }^{52}$ F. M. Brochu, ${ }^{27}$ I. Brock, ${ }^{20}$ R. Brock,${ }^{87}$ E. Brodet, ${ }^{152}$ F. Broggi, ${ }^{88 \mathrm{a}}$ C. Bromberg, ${ }^{87}$ J. Bronner, ${ }^{98}$ G. Brooijmans,${ }^{34}$ T. Brooks ${ }^{75}$ W. K. Brooks,${ }^{31 \mathrm{~b}}$ G. Brown, ${ }^{81}$ H. Brown, ${ }^{7}$

P. A. Bruckman de Renstrom,${ }^{38}$ D. Bruncko, ${ }^{143 \mathrm{~b}}$ R. Bruneliere, ${ }^{47} \mathrm{~S}$. Brunet,${ }^{59}$ A. Bruni, ${ }^{19 \mathrm{a}} \mathrm{G}$. Bruni, ${ }^{19 \mathrm{a}}$ M. Bruschi, ${ }^{19 a}$ T. Buanes, ${ }^{13}$ Q. Buat, ${ }^{54}$ F. Bucci, ${ }^{48}$ J. Buchanan, ${ }^{117}$ P. Buchholz, ${ }^{140}$ R. M. Buckingham,,${ }^{117}$ A. G. Buckley, ${ }^{45}$ S. I. Buda, ${ }^{25 a}$ I. A. Budagov, ${ }^{63}$ B. Budick, ${ }^{107}$ V. Büscher,${ }^{80}$ L. Bugge, ${ }^{116}$ O. Bulekov, ${ }^{95}$ A. C. Bundock, ${ }^{72}$ M. Bunse, ${ }^{42}$ T. Buran,,${ }^{116}$ H. Burckhart, ${ }^{29}$ S. Burdin, ${ }^{72}$ T. Burgess, ${ }^{13}$ S. Burke,,${ }^{128}$ E. Busato, ${ }^{33}$ P. Bussey, ${ }^{52}$ C. P. Buszello, ${ }^{165}$ B. Butler, ${ }^{142}$ J. M. Butler, ${ }^{21}$ C. M. Buttar, ${ }^{52}$ J. M. Butterworth, ${ }^{76}$ W. Buttinger ${ }^{27}$ S. Cabrera Urbán, ${ }^{166}$ D. Caforio, ${ }^{19 a, 19 b}$ O. Cakir, ${ }^{3 a}$ P. Calafiura, ${ }^{14}$ G. Calderini,${ }^{77}$ P. Calfayan, ${ }^{97}$ R. Calkins, ${ }^{105}$ L. P. Caloba, ${ }^{23 a}$ R. Caloi, ${ }^{131 a, 131 b}$ D. Calvet, ${ }^{33}$ S. Calvet, ${ }^{33}$ R. Camacho Toro, ${ }^{33}$ P. Camarri, ${ }^{132 a, 132 b}$ D. Cameron, ${ }^{116}$ L. M. Caminada ${ }^{14}$ S. Campana, ${ }^{29}$ M. Campanelli, ${ }^{76}$ V. Canale, ${ }^{101 a, 101 b}$ F. Canelli, ${ }^{30, h}$ A. Canepa, ${ }^{158 a}$ J. Cantero, ${ }^{79}$ R. Cantrill, ${ }^{75}$ L. Capasso, ${ }^{101 a, 101 b}$ M. D. M. Capeans Garrido, ${ }^{29}$ I. Caprini ${ }^{25 a}$ M. Caprini, ${ }^{25 a}$ D. Capriotti, ${ }^{98}$

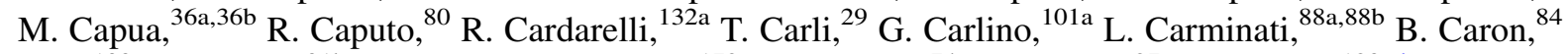
S. Caron, ${ }^{103}$ E. Carquin, ${ }^{31 \mathrm{~b}}$ G. D. Carrillo Montoya ${ }^{172}$ A. A. Carter, ${ }^{74}$ J. R. Carter, ${ }^{27}$ J. Carvalho, ${ }^{123 a, i}$ D. Casadei, ${ }^{107}$ M. P. Casado, ${ }^{11}$ M. Cascella, ${ }^{121 a, 121 b}$ C. Caso, ${ }^{49 a, 49 b, a}$ A. M. Castaneda Hernandez, ${ }^{172, j}$ E. Castaneda-Miranda, ${ }^{172}$ V. Castillo Gimenez, ${ }^{166}$ N. F. Castro, ${ }^{123 a}$ G. Cataldi, ${ }^{71 a}$ P. Catastini, ${ }^{56}$ A. Catinaccio, ${ }^{29}$ J. R. Catmore, ${ }^{29}$ A. Cattai, ${ }^{29}$

G. Cattani, ${ }^{132 a, 132 b}$ S. Caughron,${ }^{87}$ P. Cavalleri, ${ }^{77}$ D. Cavalli, ${ }^{88 a}$ M. Cavalli-Sforza, ${ }^{11}$ V. Cavasinni, ${ }^{121 a, 121 b}$ F. Ceradini, ${ }^{133 a, 133 b}$ A. S. Cerqueira, ${ }^{23 b}$ A. Cerri, ${ }^{29}$ L. Cerrito, ${ }^{74}$ F. Cerutti, ${ }^{46}$ S. A. Cetin, ${ }^{18 b}$ A. Chafaq, ${ }^{134 a}$ D. Chakraborty, ${ }^{105}$ I. Chalupkova, ${ }^{125}$ K. Chan, ${ }^{2}$ B. Chapleau, ${ }^{84}$ J. D. Chapman, ${ }^{27}$ J. W. Chapman, ${ }^{86}$ E. Chareyre, ${ }^{77}$ D. G. Charlton, ${ }^{17}$ V. Chavda,${ }^{81}$ C. A. Chavez Barajas, ${ }^{29}$ S. Cheatham,${ }^{84}$ S. Chekanov, ${ }^{5}$ S. V. Chekulaev, ${ }^{158 a}$ G. A. Chelkov, ${ }^{63}$ M. A. Chelstowska, ${ }^{103}$ C. Chen, ${ }^{62}$ H. Chen, ${ }^{24}$ S. Chen, ${ }^{32 c}$ X. Chen, ${ }^{172}$ A. Cheplakov, ${ }^{63}$ R. Cherkaoui El Moursli, ${ }^{134 \mathrm{e}}$ V. Chernyatin,${ }^{24}$ E. Cheu, ${ }^{6}$ S. L. Cheung, ${ }^{157}$ L. Chevalier, ${ }^{135}$ G. Chiefari, ${ }^{101 \mathrm{a}, 101 \mathrm{~b}}$ L. Chikovani, ${ }^{50 a, a}$ J. T. Childers, ${ }^{29}$ A. Chilingarov, ${ }^{70}$ G. Chiodini, ${ }^{71 a}$ A. S. Chisholm,,${ }^{17}$ R. T. Chislett, ${ }^{76}$

M. V. Chizhov, ${ }^{63}$ G. Choudalakis, ${ }^{30}$ S. Chouridou, ${ }^{136}$ I. A. Christidi ${ }^{76}$ A. Christov, ${ }^{47}$ D. Chromek-Burckhart, ${ }^{29}$ M. L. Chu, ${ }^{150}$ J. Chudoba, ${ }^{124}$ G. Ciapetti, ${ }^{131 a, 131 b}$ A. K. Ciftci, ${ }^{3 a}$ R. Ciftci,${ }^{3 a}$ D. Cinca, ${ }^{33}$ V. Cindro, ${ }^{73}$ C. Ciocca, ${ }^{19 a, 19 b}$ 
A. Ciocio, ${ }^{14}$ M. Cirilli, ${ }^{86}$ P. Cirkovic, ${ }^{12 b}$ M. Citterio, ${ }^{88 a}$ M. Ciubancan, ${ }^{25 a}$ A. Clark, ${ }^{48}$ P. J. Clark, ${ }^{45}$ W. Cleland, ${ }^{122}$ J. C. Clemens, ${ }^{82}$ B. Clement, ${ }^{54}$ C. Clement, ${ }^{145 a, 145 b}$ Y. Coadou, ${ }^{82}$ M. Cobal, ${ }^{163 a, 163 \mathrm{c}}$ A. Coccaro, ${ }^{137}$ J. Cochran, ${ }^{62}$ J. G. Cogan, ${ }^{142}$ J. Coggeshall, ${ }^{164}$ E. Cogneras, ${ }^{177}$ J. Colas, ${ }^{4}$ A. P. Colijn,,${ }^{104}$ N. J. Collins, ${ }^{17}$ C. Collins-Tooth, ${ }^{52}$ J. Collot, ${ }^{54}$ T. Colombo, ${ }^{118 a, 118 b}$ G. Colon, ${ }^{83}$ P. Conde Muiño, ${ }^{123 a}$ E. Coniavitis, ${ }^{117}$ M. C. Conidi, ${ }^{11}$ S. M. Consonni, ${ }^{88 a, 88 b}$ V. Consorti, ${ }^{47}$ S. Constantinescu, ${ }^{25 a}$ C. Conta, ${ }^{18 a, 118 b}$ G. Conti, ${ }^{56}$ F. Conventi, ${ }^{101 a, k}$ M. Cooke, ${ }^{14}$ B. D. Cooper ${ }^{76}$ A. M. Cooper-Sarkar, ${ }^{17}$ K. Copic, ${ }^{14}$ T. Cornelissen, ${ }^{174}$ M. Corradi, ${ }^{19 a}$ F. Corriveau, ${ }^{84,1}$ A. Cortes-Gonzalez, ${ }^{164}$ G. Cortiana,${ }^{98}$ G. Costa,${ }^{88 a}$ M. J. Costa, ${ }^{166}$ D. Costanzo, ${ }^{138}$ T. Costin, ${ }^{30}$ D. Côté, ${ }^{29}$ L. Courneyea, ${ }^{168}$ G. Cowan, ${ }^{75}$ C. Cowden, ${ }^{27}$ B. E. Cox, ${ }^{81}$ K. Cranmer, ${ }^{107}$ F. Crescioli, ${ }^{121 a, 121 b}$ M. Cristinziani, ${ }^{20}$ G. Crosetti, ${ }^{36 a, 36 b}$ R. Crupi ${ }^{71 a, 71 b}$ S. Crépé-Renaudin, ${ }^{54}$ C.-M. Cuciuc ${ }^{25 a}$ C. Cuenca Almenar, ${ }^{175}$

T. Cuhadar Donszelmann, ${ }^{138}$ M. Curatolo, ${ }^{46}$ C. J. Curtis,${ }^{17}$ C. Cuthbert, ${ }^{149}$ P. Cwetanski ${ }^{59}$ H. Czirr, ${ }^{140}$ P. Czodrowski, ${ }^{43}$ Z. Czyczula, ${ }^{175}$ S. D'Auria,${ }^{52}$ M. D'Onofrio, ${ }^{72}$ A. D'Orazio, ${ }^{131 a, 131 b}$

M. J. Da Cunha Sargedas De Sousa, ${ }^{123 a}$ C. Da Via ${ }^{81}$ W. Dabrowski, ${ }^{37}$ A. Dafinca, ${ }^{117}$ T. Dai,${ }^{86}$ C. Dallapiccola,${ }^{83}$ M. Dam,${ }^{35}$ M. Dameri, ${ }^{49 a, 49 b}$ D. S. Damiani ${ }^{136}$ H. O. Danielsson, ${ }^{29}$ V. Dao, ${ }^{48}$ G. Darbo, ${ }^{49 a}$ G. L. Darlea, ${ }^{25 b}$ W. Davey ${ }^{20}$ T. Davidek, ${ }^{125}$ N. Davidson,${ }^{85}$ R. Davidson, ${ }^{70}$ E. Davies, ${ }^{117, d}$ M. Davies, ${ }^{92}$ A. R. Davison, ${ }^{76}$ Y. Davygora ${ }^{57 a}$ E. Dawe, ${ }^{141}$ I. Dawson, ${ }^{138}$ R. K. Daya-Ishmukhametova, ${ }^{22}$ K. De, ${ }^{7}$ R. de Asmundis, ${ }^{101 a}$ S. De Castro, ${ }^{19 a, 19 b}$ S. De Cecco, ${ }^{77}$ J. de Graat,${ }^{97}$ N. De Groot,${ }^{103}$ P. de Jong, ${ }^{104}$ C. De La Taille, ${ }^{114}$ H. De la Torre, ${ }^{79}$ F. De Lorenzi, ${ }^{62}$ L. de Mora,${ }^{70}$ L. De Nooij, ${ }^{104}$ D. De Pedis, ${ }^{131 a}$ A. De Salvo, ${ }^{131 a}$ U. De Sanctis, ${ }^{163 a, 163 c}$ A. De Santo, ${ }^{148}$ J. B. De Vivie De Regie, ${ }^{114}$ G. De Zorzi, ${ }^{131 a, 131 b}$ W. J. Dearnaley, ${ }^{70}$ R. Debbe,${ }^{24}$ C. Debenedetti,${ }^{45}$ B. Dechenaux ${ }^{54}$ D. V. Dedovich, ${ }^{63}$ J. Degenhardt, ${ }^{119}$ C. Del Papa, ${ }^{163 a, 163 c}$ J. Del Peso, ${ }^{79}$ T. Del Prete, ${ }^{121 a, 121 b}$ T. Delemontex,${ }^{54}$ M. Deliyergiyever ${ }^{73}$ A. Dell'Acqua,${ }^{29}$ L. Dell' Asta, ${ }^{21}$ M. Della Pietra, ${ }^{101 \mathrm{a}, \mathrm{k}}$ D. della Volpe,${ }^{101 \mathrm{a}, 101 \mathrm{~b}}$ M. Delmastro, ${ }^{4}$ P. A. Delsart, ${ }^{54}$ C. Deluca, ${ }^{104}$ S. Demers,${ }^{175}$ M. Demichev, ${ }^{63}$ B. Demirkoz,${ }^{11, m}$ J. Deng, ${ }^{162}$ S. P. Denisov, ${ }^{127}$ D. Derendarz, ${ }^{38}$ J. E. Derkaoui, ${ }^{134 \mathrm{~d}}$ F. Derue,${ }^{77}$ P. Dervan,${ }^{72}$ K. Desch,${ }^{20}$ E. Devetak,${ }^{147}$ P. O. Deviveiros, ${ }^{104}$ A. Dewhurst, ${ }^{128}$ B. DeWilde, ${ }^{147}$ S. Dhaliwal, ${ }^{157}$ R. Dhullipudi, ${ }^{24, n}$ A. Di Ciaccio, ${ }^{132 a, 132 b}$ L. Di Ciaccio, ${ }^{4}$ A. Di Girolamo, ${ }^{29}$ B. Di Girolamo, ${ }^{29}$ S. Di Luise,${ }^{133 a, 133 b}$ A. Di Mattia, ${ }^{172}$ B. Di Micco, ${ }^{29}$ R. Di Nardo ${ }^{46}$ A. Di Simone, ${ }^{132 a, 132 b}$ R. Di Sipio, ${ }^{19 a, 19 b}$ M. A. Diaz, ${ }^{31 a}$ E. B. Diehl, ${ }^{86}$ J. Dietrich, ${ }^{41}$ T. A. Dietzsch, ${ }^{57 a}$ S. Diglio, ${ }^{85}$ K. Dindar Yagci, ${ }^{39}$ J. Dingfelder, ${ }^{20}$ C. Dionisi, ${ }^{131 a, 131 b}$ P. Dita, ${ }^{25 a}$ S. Dita, ${ }^{25 a}$ F. Dittus, ${ }^{29}$ F. Djama, ${ }^{82}$ T. Djobava, ${ }^{50 b}$ M. A. B. do Vale, ${ }^{23 c}$ A. Do Valle Wemans, ${ }^{123 a, o}$ T. K. O. Doan, ${ }^{4}$ M. Dobbs,${ }^{84}$ R. Dobinson, ${ }^{29, a}$ D. Dobos, ${ }^{29}$ E. Dobson, ${ }^{29, p}$ J. Dodd,${ }^{34}$ C. Doglioni,${ }^{48}$ T. Doherty, ${ }^{52}$ Y. Doi,${ }^{64, a}$ J. Dolejsi,${ }^{125}$ I. Dolenc, ${ }^{73}$ Z. Dolezal, ${ }^{125}$ B. A. Dolgoshein, ${ }^{95, a}$ T. Dohmae,${ }^{154}$ M. Donadelli, ${ }^{23 d}$ M. Donega,${ }^{119}$ J. Donini, ${ }^{33}$ J. Dopke, ${ }^{29}$ A. Doria, ${ }^{101 \mathrm{a}}$ A. Dos Anjos, ${ }^{172}$ A. Dotti, ${ }^{121 \mathrm{a}, 121 \mathrm{~b}}$ M. T. Dova, ${ }^{69}$ A. D. Doxiadis, ${ }^{104}$ A. T. Doyle, ${ }^{52}$ M. Dris,${ }^{9}$ J. Dubbert ${ }^{98}$ S. Dube,${ }^{14}$ E. Duchovni, ${ }^{171}$ G. Duckeck, ${ }^{97}$ A. Dudarev, ${ }^{29}$ F. Dudziak,${ }^{62}$ M. Dührssen, ${ }^{29}$ I. P. Duerdoth, ${ }^{81}$

L. Duflot, ${ }^{114}$ M-A. Dufour, ${ }^{84}$ M. Dunford ${ }^{29}$ H. Duran Yildiz, ${ }^{3 a}$ R. Duxfield, ${ }^{138}$ M. Dwuznik, ${ }^{37}$ F. Dydak,${ }^{29}$ M. Düren,${ }^{51}$ J. Ebke, ${ }^{97}$ S. Eckweiler, ${ }^{80}$ K. Edmonds,${ }^{80}$ C. A. Edwards, ${ }^{75}$ N. C. Edwards,${ }^{52}$ W. Ehrenfeld,${ }^{41}$ T. Eifert, ${ }^{142}$ G. Eigen, ${ }^{13}$ K. Einsweiler, ${ }^{14}$ E. Eisenhandler, ${ }^{74}$ T. Ekelof, ${ }^{165}$ M. El Kacimi, ${ }^{134 c}$ M. Ellert,${ }^{165}$ S. Elles, ${ }^{4}$ F. Ellinghaus,${ }^{80}$ K. Ellis, ${ }^{74}$ N. Ellis, ${ }^{29}$ J. Elmsheuser,${ }^{97}$ M. Elsing, ${ }^{29}$ D. Emeliyanov, ${ }^{128}$ R. Engelmann, ${ }^{147}$ A. Engl,${ }^{97}$

B. Epp ${ }^{60}$ A. Eppig,${ }^{86}$ J. Erdmann, ${ }^{53}$ A. Ereditato,${ }^{16}$ D. Eriksson, ${ }^{145 a}$ J. Ernst, ${ }^{1}$ M. Ernst, ${ }^{24}$ J. Ernwein, ${ }^{135}$

D. Errede,${ }^{164}$ S. Errede, ${ }^{164}$ E. Ertel,${ }^{80}$ M. Escalier, ${ }^{114}$ C. Escobar, ${ }^{122}$ X. Espinal Curull, ${ }^{11}$ B. Esposito, ${ }^{46}$ F. Etienne, ${ }^{82}$ A. I. Etienvre, ${ }^{135}$ E. Etzion, ${ }^{152}$ D. Evangelakou, ${ }^{53}$ H. Evans, ${ }^{59}$ L. Fabbri, ${ }^{19 a},{ }^{19 b}$ C. Fabre,${ }^{29}$ R. M. Fakhrutdinov, ${ }^{127}$ S. Falciano, ${ }^{131 a}$ Y. Fang, ${ }^{172}$ M. Fanti ${ }^{88 a, 88 b}$ A. Farbin, ${ }^{7}$ A. Farilla, ${ }^{133 a}$ J. Farley, ${ }^{147}$ T. Farooque, ${ }^{157}$ S. Farrell, ${ }^{162}$

S. M. Farrington, ${ }^{117}$ P. Farthouat, ${ }^{29}$ P. Fassnacht, ${ }^{29}$ D. Fassouliotis, ${ }^{8}$ B. Fatholahzadeh, ${ }^{157}$ A. Favareto, ${ }^{88 a, 88 b}$ L. Fayard, ${ }^{114}$ S. Fazio, ${ }^{36 a, 36 b}$ R. Febbraro, ${ }^{33}$ P. Federic,,${ }^{143 a}$ O. L. Fedin, ${ }^{120}$ W. Fedorko ${ }^{87}$ M. Fehling-Kaschek, ${ }^{47}$ L. Feligioni, ${ }^{82}$ D. Fellmann, ${ }^{5}$ C. Feng, ${ }^{32 \mathrm{~d}}$ E. J. Feng, ${ }^{30}$ A. B. Fenyuk, ${ }^{127}$ J. Ferencei, ${ }^{143 \mathrm{~b}}$ W. Fernando,${ }^{5}$ S. Ferrag ${ }^{52}$ J. Ferrando, ${ }^{52}$ V. Ferrara, ${ }^{41}$ A. Ferrari, ${ }^{165}$ P. Ferrari, ${ }^{104}$ R. Ferrari, ${ }^{118 a}$ D. E. Ferreira de Lima, ${ }^{52}$ A. Ferrer, ${ }^{166}$ D. Ferrere, ${ }^{48}$ C. Ferretti, ${ }^{86}$ A. Ferretto Parodi,${ }^{49 a, 49 b}$ M. Fiascaris,${ }^{30}$ F. Fiedler ${ }^{80}$ A. Filipčič,${ }^{73}$ F. Filthaut, ${ }^{103}$ M. Fincke-Keeler, ${ }^{168}$ M. C. N. Fiolhais, ${ }^{123 a, i}$ L. Fiorini, ${ }^{166}$ A. Firan, ${ }^{39}$ G. Fischer, ${ }^{41}$ M. J. Fisher, ${ }^{108}$ M. Flechl, ${ }^{47}$ I. Fleck, ${ }^{140}$ J. Fleckner, ${ }^{80}$ P. Fleischmann, ${ }^{173}$ S. Fleischmann, ${ }^{174}$ T. Flick, ${ }^{174}$ A. Floderus, ${ }^{78}$ L. R. Flores Castillo, ${ }^{172}$ M. J. Flowerdew, ${ }^{98}$ T. Fonseca Martin, ${ }^{16}$ A. Formica, ${ }^{135}$ A. Forti, ${ }^{81}$ D. Fortin, ${ }^{158 a}$ D. Fournier, ${ }^{114}$ H. Fox,${ }^{70}$ P. Francavilla, ${ }^{11}$ S. Franchino, ${ }^{118 a, 118 b}$ D. Francis,${ }^{29}$ T. Frank,${ }^{171}$ S. Franz, ${ }^{29}$ M. Fraternali, ${ }^{118 a, 118 b}$ S. Fratina ${ }^{119}$

S. T. French,${ }^{27}$ C. Friedrich,${ }^{41}$ F. Friedrich, ${ }^{43}$ R. Froeschl,${ }^{29}$ D. Froidevaux,${ }^{29}$ J. A. Frost, ${ }^{27}$ C. Fukunaga,${ }^{155}$ E. Fullana Torregrosa, ${ }^{29}$ B. G. Fulsom, ${ }^{142}$ J. Fuster, ${ }^{166}$ C. Gabaldon, ${ }^{29}$ O. Gabizon, ${ }^{171}$ T. Gadfort ${ }^{24}$ S. Gadomski, ${ }^{48}$ G. Gagliardi, ${ }^{49 a, 49 b}$ P. Gagnon, ${ }^{59}$ C. Galea, ${ }^{97}$ E. J. Gallas, ${ }^{117}$ V. Gallo, ${ }^{16}$ B. J. Gallop, ${ }^{128}$ P. Gallus, ${ }^{124}$ K. K. Gan, ${ }^{108}$ 
Y. S. Gao, ${ }^{142, f}$ A. Gaponenko, ${ }^{14}$ F. Garberson, ${ }^{175}$ M. Garcia-Sciveres, ${ }^{14}$ C. García, ${ }^{166}$ J. E. García Navarro, ${ }^{166}$ R. W. Gardner ${ }^{30}$ N. Garelli ${ }^{29}$ H. Garitaonandia, ${ }^{104}$ V. Garonne,${ }^{29}$ J. Garvey, ${ }^{17}$ C. Gatti, ${ }^{46}$ G. Gaudio, ${ }^{118 a}$ B. Gaur, ${ }^{140}$ L. Gauthier, ${ }^{135}$ P. Gauzzi, ${ }^{131 \mathrm{a}, 131 \mathrm{~b}}$ I. L. Gavrilenko, ${ }^{93}$ C. Gay, ${ }^{167}$ G. Gaycken,${ }^{20}$ E. N. Gazis, ${ }^{9}$ P. Ge, ${ }^{32 \mathrm{~d}}$ Z. Gecse, ${ }^{167}$ C. N. P. Gee, ${ }^{128}$ D. A. A. Geerts, ${ }^{104}$ Ch. Geich-Gimbel, ${ }^{20}$ K. Gellerstedt, ${ }^{145 a, 145 b}$ C. Gemme, ${ }^{49 a}$ A. Gemmell, ${ }^{52}$ M. H. Genest, ${ }^{54}$ S. Gentile, ${ }^{131 a, 131 b}$ M. George, ${ }^{53}$ S. George, ${ }^{75}$ P. Gerlach, ${ }^{174}$ A. Gershon, ${ }^{152}$ C. Geweniger, ${ }^{57 a}$ H. Ghazlane, ${ }^{134 b}$ N. Ghodbane, ${ }^{33}$ B. Giacobbe, ${ }^{19 a}$ S. Giagu, ${ }^{131 a, 131 b}$ V. Giakoumopoulou, ${ }^{8}$ V. Giangiobbe, ${ }^{11}$ F. Gianotti ${ }^{29}$ B. Gibbard ${ }^{24}$ A. Gibson, ${ }^{157}$ S. M. Gibson, ${ }^{29}$ D. Gillberg, ${ }^{28}$ A. R. Gillman, ${ }^{128}$ D. M. Gingrich, ${ }^{2, e}$ J. Ginzburg, ${ }^{152}$ N. Giokaris, ${ }^{8}$ M. P. Giordani, ${ }^{163 \mathrm{c}}$ R. Giordano, ${ }^{101 \mathrm{a}, 101 \mathrm{~b}}$ F. M. Giorgi, ${ }^{15}$ P. Giovannini, ${ }^{98}$ P. F. Giraud,${ }^{135}$ D. Giugni, ${ }^{88 a}$ M. Giunta, ${ }^{92}$ P. Giusti, ${ }^{19 a}$ B. K. Gjelsten, ${ }^{116}$ L. K. Gladilin, ${ }^{96}$ C. Glasman, ${ }^{79}$ J. Glatzer, ${ }^{47}$ A. Glazov, ${ }^{41}$

K. W. Glitza, ${ }^{174}$ G. L. Glonti, ${ }^{63}$ J. R. Goddard, ${ }^{74}$ J. Godfrey, ${ }^{141}$ J. Godlewski, ${ }^{29}$ M. Goebel, ${ }^{41}$ T. Göpfert, ${ }^{43}$ C. Goeringer ${ }^{80}$ C. Gössling, ${ }^{42}$ S. Goldfarb, ${ }^{86}$ T. Golling, ${ }^{175}$ A. Gomes, ${ }^{123 a, c}$ L. S. Gomez Fajardo, ${ }^{41}$ R. Gonçalo, ${ }^{75}$ J. Goncalves Pinto Firmino Da Costa, ${ }^{41}$ L. Gonella, ${ }^{20}$ S. Gonzalez, ${ }^{172}$ S. González de la Hoz, ${ }^{166}$ G. Gonzalez Parra, ${ }^{11}$ M. L. Gonzalez Silva, ${ }^{26}$ S. Gonzalez-Sevilla, ${ }^{48}$ J. J. Goodson, ${ }^{147}$ L. Goossens,${ }^{29}$ P. A. Gorbounov, ${ }^{94}$ H. A. Gordon, ${ }^{24}$ I. Gorelov, ${ }^{102}$ G. Gorfine, ${ }^{174}$ B. Gorini, ${ }^{29}$ E. Gorini, ${ }^{71 a, 71 b}$ A. Gorišek, ${ }^{73}$ E. Gornicki, ${ }^{38}$ B. Gosdzik, ${ }^{41}$ A. T. Goshaw, M. Gosselink, ${ }^{104}$ M. I. Gostkin, ${ }^{63}$ I. Gough Eschrich, ${ }^{162}$ M. Gouighri, ${ }^{134 a}$ D. Goujdami, ${ }^{134 c}$ M. P. Goulette, ${ }^{48}$ A. G. Goussiou, ${ }^{137}$ C. Goy, ${ }^{4}$ S. Gozpinar, ${ }^{22}$ I. Grabowska-Bold, ${ }^{37}$ P. Grafström, ${ }^{29}$ K-J. Grahn, ${ }^{41}$ F. Grancagnolo, ${ }^{71 a}$ S. Grancagnolo, ${ }^{15}$ V. Grassi, ${ }^{147}$ V. Gratchev, ${ }^{120}$ N. Grau,${ }^{34}$ H. M. Gray, ${ }^{29}$ J. A. Gray, ${ }^{147}$ E. Graziani, ${ }^{133 a}$

O. G. Grebenyuk, ${ }^{120}$ T. Greenshaw, ${ }^{72}$ Z. D. Greenwood, ${ }^{24, n}$ K. Gregersen,${ }^{35}$ I. M. Gregor, ${ }^{41}$ P. Grenier, ${ }^{142}$ J. Griffiths, ${ }^{137}$ N. Grigalashvili, ${ }^{63}$ A. A. Grillo, ${ }^{136}$ S. Grinstein, ${ }^{11}$ Y. V. Grishkevich, ${ }^{96}$ J.-F. Grivaz, ${ }^{114}$ E. Gross, ${ }^{171}$ J. Grosse-Knetter, ${ }^{53}$ J. Groth-Jensen, ${ }^{171} \mathrm{~K}$. Grybel,${ }^{140}$ D. Guest, ${ }^{175}$ C. Guicheney, ${ }^{33}$ A. Guida, ${ }^{71 a, 71 b}$ S. Guindon, ${ }^{53}$ U. Gul ${ }^{52}$ H. Guler ${ }^{84, q}$ J. Gunther, ${ }^{124}$ B. Guo, ${ }^{157}$ J. Guo, ${ }^{34}$ P. Gutierrez, ${ }^{110}$ N. Guttman, ${ }^{152}$ O. Gutzwiller, ${ }^{172}$ C. Guyot, ${ }^{135}$ C. Gwenlan, ${ }^{117}$ C. B. Gwilliam, ${ }^{72}$ A. Haas, ${ }^{142}$ S. Haas,${ }^{29}$ C. Haber, ${ }^{14}$ H. K. Hadavand,${ }^{39}$ D. R. Hadley, ${ }^{17}$ P. Haefner, ${ }^{20}$ F. Hahn,${ }^{29}$ S. Haider, ${ }^{29}$ Z. Hajduk ${ }^{38}$ H. Hakobyan, ${ }^{176}$ D. Hall, ${ }^{117}$ J. Haller, ${ }^{53}$ K. Hamacher, ${ }^{174}$ P. Hamal, ${ }^{112}$ M. Hamer, ${ }^{53}$ A. Hamilton, ${ }^{144 b, r}$ S. Hamilton, ${ }^{160}$ L. Han,${ }^{32 b}$ K. Hanagaki, ${ }^{115}$ K. Hanawa, ${ }^{159}$ M. Hance, ${ }^{14}$ C. Handel, ${ }^{80}$ P. Hanke, ${ }^{57 a}$ J. R. Hansen, ${ }^{35}$ J. B. Hansen, ${ }^{35}$ J. D. Hansen, ${ }^{35}$ P. H. Hansen, ${ }^{35}$ P. Hansson, ${ }^{142}$ K. Hara, ${ }^{159}$ G. A. Hare, ${ }^{136}$ T. Harenberg, ${ }^{174}$ S. Harkusha, ${ }^{89}$ D. Harper, ${ }^{86}$ R. D. Harrington, ${ }^{45}$ O. M. Harris, ${ }^{137}$ K. Harrison, ${ }^{17}$ J. Hartert, ${ }^{47}$ F. Hartjes, ${ }^{104}$ T. Haruyama, ${ }^{64}$ A. Harvey, ${ }^{55}$ S. Hasegawa, ${ }^{100}$ Y. Hasegawa, ${ }^{139}$ S. Hassani, ${ }^{135}$ S. Haug ${ }^{16}$ M. Hauschild, ${ }^{29}$ R. Hauser, ${ }^{87}$ M. Havranek, ${ }^{20}$ C. M. Hawkes, ${ }^{17}$ R. J. Hawkings, ${ }^{29}$ A. D. Hawkins, ${ }^{78}$ D. Hawkins, ${ }^{162}$ T. Hayakawa, ${ }^{65}$ T. Hayashi, ${ }^{159}$ D. Hayden, ${ }^{75}$ C. P. Hays, ${ }^{117}$ H. S. Hayward, ${ }^{72}$ S. J. Haywood, ${ }^{128}$ M. He, ${ }^{32 d}$

S. J. Head,${ }^{17}$ V. Hedberg ${ }^{78}$ L. Heelan, ${ }^{7}$ S. Heim,${ }^{87}$ B. Heinemann, ${ }^{14}$ S. Heisterkamp, ${ }^{35}$ L. Helary, ${ }^{4}$ C. Heller,${ }^{97}$ M. Heller ${ }^{29}$ S. Hellman, ${ }^{145 a, 145 b}$ D. Hellmich, ${ }^{20}$ C. Helsens,${ }^{11}$ R. C. W. Henderson, ${ }^{70}$ M. Henke, ${ }^{57 a}$ A. Henrichs, ${ }^{53}$ A. M. Henriques Correia, ${ }^{29}$ S. Henrot-Versille, ${ }^{114}$ C. Hensel,${ }^{53}$ T. Hen $\beta,{ }^{174}$ C. M. Hernandez, ${ }^{7}$

Y. Hernández Jiménez, ${ }^{166}$ R. Herrberg,${ }^{15}$ G. Herten, ${ }^{47}$ R. Hertenberger ${ }^{97}$ L. Hervas, ${ }^{29}$ G. G. Hesketh,${ }^{76}$ N.P. Hessey, ${ }^{104}$ E. Higón-Rodriguez, ${ }^{166}$ J. C. Hill, ${ }^{27}$ K. H. Hiller, ${ }^{41}$ S. Hillert, ${ }^{20}$ S. J. Hillier, ${ }^{17}$ I. Hinchliffe, ${ }^{14}$ E. Hines,${ }^{119}$ M. Hirose, ${ }^{115}$ F. Hirsch, ${ }^{42}$ D. Hirschbuehl, ${ }^{174}$ J. Hobbs, ${ }^{147}$ N. Hod,${ }^{152}$ M. C. Hodgkinson, ${ }^{138}$ P. Hodgson, ${ }^{138}$ A. Hoecker, ${ }^{29}$ M. R. Hoeferkamp, ${ }^{102}$ J. Hoffman, ${ }^{39}$ D. Hoffmann, ${ }^{82}$ M. Hohlfeld, ${ }^{80}$ M. Holder, ${ }^{140}$ S. O. Holmgren, ${ }^{145 a}$ T. Holy, ${ }^{126}$ J. L. Holzbauer, ${ }^{87}$ T. M. Hong, ${ }^{119}$ L. Hooft van Huysduynen, ${ }^{107}$ C. Horn, ${ }^{142}$ S. Horner ${ }^{47}$ J-Y. Hostachy, ${ }^{54}$ S. Hou,${ }^{150}$ A. Hoummada, ${ }^{134 a}$ J. Howard, ${ }^{117}$ J. Howarth, ${ }^{81}$ I. Hristova, ${ }^{15}$ J. Hrivnac, ${ }^{114}$ T. Hryn'ova, ${ }^{4}$ P. J. Hsu, ${ }^{80}$ S.-C. Hsu, ${ }^{14}$ Z. Hubacek, ${ }^{126}$ F. Hubaut, ${ }^{82}$ F. Huegging, ${ }^{20}$ A. Huettmann, ${ }^{41}$ T. B. Huffman, ${ }^{117}$ E. W. Hughes, ${ }^{34}$ G. Hughes,${ }^{70}$ M. Huhtinen,${ }^{29}$ M. Hurwitz, ${ }^{14}$ U. Husemann, ${ }^{41}$ N. Huseynov, ${ }^{63, s}$ J. Huston, ${ }^{87}$ J. Huth, ${ }^{56}$ G. Iacobucci, ${ }^{48}$ G. Iakovidis, ${ }^{9}$ M. Ibbotson, ${ }^{81}$ I. Ibragimov ${ }^{140}$ L. Iconomidou-Fayard, ${ }^{114}$ J. Idarraga ${ }^{114}{ }^{14}$. Iengo, ${ }^{101 a}$ O. Igonkina, ${ }^{104}$ Y. Ikegami,${ }^{64} \mathrm{M}$. Ikeno,${ }^{64} \mathrm{D}$. Iliadis, ${ }^{153} \mathrm{~N}$. Ilic, ${ }^{157} \mathrm{~T}$. Ince, ${ }^{20} \mathrm{~J}$. Inigo-Golfin, ${ }^{29} \mathrm{P}$. Ioannou, ${ }^{8}$ M. Iodice, ${ }^{133 a} \mathrm{~K}$. Iordanidou, ${ }^{8} \mathrm{~V}$. Ippolito, ${ }^{131 \mathrm{a}, 131 \mathrm{~b}} \mathrm{~A}$. Irles Quiles, ${ }^{166} \mathrm{C}$. Isaksson, ${ }^{165} \mathrm{~A}$. Ishikawa, ${ }^{65} \mathrm{M}$. Ishino, ${ }^{66}$ R. Ishmukhametov, ${ }^{39}$ C. Issever, ${ }^{117}$ S. Istin, ${ }^{18 a}$ A. V. Ivashin, ${ }^{127}$ W. Iwanski, ${ }^{38}$ H. Iwasaki, ${ }^{64} \mathrm{~J}$. M. Izen,${ }^{40}$ V. Izzo, ${ }^{101 a}$ B. Jackson, ${ }^{119}$ J. N. Jackson, ${ }^{72}$ P. Jackson, ${ }^{142}$ M. R. Jaekel, ${ }^{29}$ V. Jain, ${ }^{59}$ K. Jakobs, ${ }^{47}$ S. Jakobsen, ${ }^{35}$ T. Jakoubek, ${ }^{124}$ J. Jakubek, ${ }^{126}$ D. K. Jana, ${ }^{110}$ E. Jansen, ${ }^{76}$ H. Jansen, ${ }^{29}$ A. Jantsch, ${ }^{98}$ M. Janus, ${ }^{47}$ G. Jarlskog, ${ }^{78}$ L. Jeanty, ${ }^{56}$ I. Jen-La Plante, ${ }^{30}$ P. Jenni, ${ }^{29}$ A. Jeremie, ${ }^{4}$ P. Jež, ${ }^{35}$ S. Jézéquel, ${ }^{4}$ M. K. Jha, ${ }^{19 a} \mathrm{H}$. Ji, ${ }^{172}$ W. Ji, ${ }^{80}$ J. Jia, ${ }^{147}$ Y. Jiang, ${ }^{32 b}$ M. Jimenez Belenguer, ${ }^{41}$ S. Jin ${ }^{32 a}$ O. Jinnouchi, ${ }^{156}$ M. D. Joergensen, ${ }^{35}$ D. Joffe, ${ }^{39}$ M. Johansen, ${ }^{145 a, 145 b}$

K. E. Johansson, ${ }^{145 a}$ P. Johansson, ${ }^{138}$ S. Johnert, ${ }^{41}$ K. A. Johns, ${ }^{6}$ K. Jon-And,${ }^{145 a, 145 b}$ G. Jones,${ }^{169}$ R. W. L. Jones, ${ }^{70}$

T. J. Jones, ${ }^{72}$ C. Joram,${ }^{29}$ P. M. Jorge, ${ }^{123 a}$ K. D. Joshi, ${ }^{81}$ J. Jovicevic, ${ }^{146}$ T. Jovin, ${ }^{12 b}$ X. Ju, ${ }^{172}$ C. A. Jung, ${ }^{42}$ R. M. Jungst,${ }^{29}$ V. Juranek, ${ }^{124}$ P. Jussel,${ }^{60}$ A. Juste Rozas, ${ }^{11}$ S. Kabana,${ }^{16}$ M. Kaci, ${ }^{166}$ A. Kaczmarska, ${ }^{38}$ P. Kadlecik, ${ }^{35}$ 
M. Kado, ${ }^{114}$ H. Kagan, ${ }^{108}$ M. Kagan ${ }^{56}$ E. Kajomovitz, ${ }^{151}$ S. Kalinin, ${ }^{174}$ L. V. Kalinovskaya, ${ }^{63}$ S. Kama, ${ }^{39}$ N. Kanaya ${ }^{154}$ M. Kaneda, ${ }^{29}$ S. Kaneti ${ }^{27}$ T. Kanno, ${ }^{156}$ V. A. Kantserov, ${ }^{95}$ J. Kanzaki, ${ }^{64}$ B. Kaplan, ${ }^{175}$ A. Kapliy, ${ }^{30}$ J. Kaplon, ${ }^{29}$ D. Kar, ${ }^{52}$ M. Karagounis, ${ }^{20}$ K. Karakostas, ${ }^{9}$ M. Karnevskiy, ${ }^{41}$ V. Kartvelishvili, ${ }^{70}$ A. N. Karyukhin, ${ }^{127}$ L. Kashif, ${ }^{172}$ G. Kasieczka, ${ }^{57 b}$ R. D. Kass, ${ }^{108}$ A. Kastanas, ${ }^{13}$ M. Kataoka, ${ }^{4}$ Y. Kataoka, ${ }^{154}$ E. Katsoufis, ${ }^{9}$ J. Katzy, ${ }^{41}$ V. Kaushik, ${ }^{6}$ K. Kawagoe, ${ }^{68}$ T. Kawamoto, ${ }^{154}$ G. Kawamura, ${ }^{80}$ M. S. Kayl, ${ }^{104}$ V. A. Kazanin, ${ }^{106}$ M. Y. Kazarinov, ${ }^{63}$ R. Keeler, ${ }^{168}$ R. Kehoe, ${ }^{39}$ M. Keil,${ }^{53}$ G. D. Kekelidze, ${ }^{63}$ J. S. Keller, ${ }^{137}$ M. Kenyon, ${ }^{52}$ O. Kepka, ${ }^{124}$ N. Kerschen, ${ }^{29}$ B. P. Kerševan, ${ }^{73}$ S. Kersten, ${ }^{174}$ K. Kessoku, ${ }^{154}$ J. Keung, ${ }^{157}$ F. Khalil-zada, ${ }^{10}$ H. Khandanyan, ${ }^{164}$ A. Khanov, ${ }^{111}$ D. Kharchenko, ${ }^{63}$ A. Khodinov, ${ }^{95}$ A. Khomich, ${ }^{57 a}$ T. J. Khoo, ${ }^{27}$ G. Khoriauli, ${ }^{20}$ A. Khoroshilov, ${ }^{174}$ V. Khovanskiy, ${ }^{94}$ E. Khramov ${ }^{63}$ J. Khubua, ${ }^{50 b}$ H. Kim, ${ }^{145 a, 145 b}$ M. S. Kim,${ }^{2}$ S. H. Kim, ${ }^{159}$ N. Kimura, ${ }^{170}$ O. Kind, ${ }^{15}$ B. T. King, ${ }^{72}$ M. King, ${ }^{65}$ R. S. B. King, ${ }^{117}$ J. Kirk, ${ }^{128}$ A. E. Kiryunin, ${ }^{98}$ T. Kishimoto, ${ }^{65}$ D. Kisielewska, ${ }^{37}$ T. Kittelmann, ${ }^{122}$

E. Kladiva, ${ }^{143 b}$ M. Klein, ${ }^{72}$ U. Klein, ${ }^{72}$ K. Kleinknecht, ${ }^{80}$ M. Klemetti, ${ }^{84}$ A. Klier, ${ }^{171}$ P. Klimek, ${ }^{145 a, 145 b}$ A. Klimentov, ${ }^{24}$ R. Klingenberg, ${ }^{42}$ J. A. Klinger, ${ }^{81}$ E. B. Klinkby, ${ }^{35}$ T. Klioutchnikova, ${ }^{29}$ P. F. Klok, ${ }^{103}$ S. Klous, ${ }^{104}$ E.-E. Kluge, ${ }^{57 a}$ T. Kluge, ${ }^{72}$ P. Kluit, ${ }^{104}$ S. Kluth, ${ }^{98}$ N. S. Knecht, ${ }^{157}$ E. Kneringer,${ }^{60}$ E. B. F. G. Knoops,${ }^{82}$ A. Knue, ${ }^{53}$ B. R. Ko, ${ }^{44}$ T. Kobayashi, ${ }^{154}$ M. Kobel,${ }^{43}$ M. Kocian, ${ }^{142}$ P. Kodys, ${ }^{125}$ K. Köneke, ${ }^{29}$ A. C. König, ${ }^{103}$ S. Koenig, ${ }^{80}$

L. Köpke, ${ }^{80}$ F. Koetsveld, ${ }^{103}$ P. Koevesarki, ${ }^{20}$ T. Koffas, ${ }^{28}$ E. Koffeman, ${ }^{104}$ L. A. Kogan, ${ }^{117}$ S. Kohlmann, ${ }^{174}$

F. Kohn, ${ }^{53}$ Z. Kohout, ${ }^{126}$ T. Kohriki, ${ }^{64}$ T. Koi, ${ }^{142}$ G. M. Kolachev, ${ }^{106, a}$ H. Kolanoski, ${ }^{15}$ V. Kolesnikov, ${ }^{63}$

I. Koletsou, ${ }^{88 \mathrm{a}}$ J. Koll, ${ }^{87}$ M. Kollefrath, ${ }^{47}$ A. A. Komar, ${ }^{93}$ Y. Komori, ${ }^{154}$ T. Kondo, ${ }^{64}$ T. Kono, ${ }^{41, t}$ A. I. Kononov, ${ }^{47}$ R. Konoplich, ${ }^{107, \mathrm{u}}$ N. Konstantinidis, ${ }^{76}$ S. Koperny, ${ }^{37}$ K. Korcyl, ${ }^{38}$ K. Kordas, ${ }^{153}$ A. Korn, ${ }^{117}$ A. Korol, ${ }^{106}$

I. Korolkov, ${ }^{11}$ E. V. Korolkova, ${ }^{138}$ V. A. Korotkov, ${ }^{127}$ O. Kortner, ${ }^{98}$ S. Kortner,${ }^{98}$ V. V. Kostyukhin, ${ }^{20}$ S. Kotov, ${ }^{98}$

V. M. Kotov, ${ }^{63}$ A. Kotwal,${ }^{44}$ C. Kourkoumelis,${ }^{8}$ V. Kouskoura, ${ }^{153}$ A. Koutsman, ${ }^{158 a}$ R. Kowalewski, ${ }^{168}$

T.Z. Kowalski, ${ }^{37}$ W. Kozanecki, ${ }^{135}$ A. S. Kozhin, ${ }^{127}$ V. Kral, ${ }^{126}$ V. A. Kramarenko, ${ }^{96}$ G. Kramberger,${ }^{73}$ M. W. Krasny ${ }^{77}$ A. Krasznahorkay, ${ }^{107}$ J. Kraus,${ }^{87}$ J. K. Kraus, ${ }^{20}$ S. Kreiss ${ }^{107}$ F. Krejci, ${ }^{126}$ J. Kretzschmar, ${ }^{72}$ N. Krieger, ${ }^{53}$ P. Krieger, ${ }^{157}$ K. Kroeninger ${ }^{53}$ H. Kroha, ${ }^{98}$ J. Kroll, ${ }^{119}$ J. Kroseberg, ${ }^{20}$ J. Krstic, ${ }^{12 a}$ U. Kruchonak, ${ }^{63}$ H. Krüger, ${ }^{20}$ T. Kruker, ${ }^{16}$ N. Krumnack, ${ }^{62}$ Z. V. Krumshteyn, ${ }^{63}$ A. Kruth, ${ }^{20}$ T. Kubota, ${ }^{85}$ S. Kuday, ${ }^{3 a}$ S. Kuehn,${ }^{47}$ A. Kugel, ${ }^{57 \mathrm{c}}$ T. Kuhl,${ }^{41}$ D. Kuhn, ${ }^{60}$ V. Kukhtin, ${ }^{63}$ Y. Kulchitsky, ${ }^{89}$ S. Kuleshov, ${ }^{31 b}$ C. Kummer, ${ }^{97}$ M. Kuna, ${ }^{77}$

J. Kunkle, ${ }^{119}$ A. Kupco, ${ }^{124}$ H. Kurashige, ${ }^{65}$ M. Kurata, ${ }^{159}$ Y. A. Kurochkin, ${ }^{89}$ V. Kus, ${ }^{124}$ E. S. Kuwertz, ${ }^{146}$ M. Kuze,${ }^{156}$ J. Kvita, ${ }^{141}$ R. Kwee, ${ }^{15}$ A. La Rosa,${ }^{48}$ L. La Rotonda,${ }^{36 a, 36 b}$ L. Labarga, ${ }^{79}$ J. Labbe, ${ }^{4}$ S. Lablak, ${ }^{134 a}$ C. Lacasta, ${ }^{166}$ F. Lacava, ${ }^{131 a, 131 b}$ H. Lacker, ${ }^{15}$ D. Lacour, ${ }^{77}$ V. R. Lacuesta, ${ }^{166}$ E. Ladygin, ${ }^{63}$ R. Lafaye,${ }^{4}$ B. Laforge,${ }^{77}$ T. Lagouri, ${ }^{79}$ S. Lai, ${ }^{47}$ E. Laisne,${ }^{54}$ M. Lamanna, ${ }^{29}$ L. Lambourne, ${ }^{76}$ C. L. Lampen, ${ }^{6}$ W. Lampl,${ }^{6}$ E. Lancon, ${ }^{135}$

U. Landgraf, ${ }^{47}$ M. P. J. Landon, ${ }^{74}$ J. L. Lane, ${ }^{81}$ C. Lange, ${ }^{41}$ A. J. Lankford, ${ }^{162}$ F. Lanni, ${ }^{24}$ K. Lantzsch,,${ }^{174}$ S. Laplace, ${ }^{77}$ C. Lapoire, ${ }^{20}$ J. F. Laporte, ${ }^{135}$ T. Lari, ${ }^{88 \mathrm{a}}$ A. Larner, ${ }^{117}$ M. Lassnig, ${ }^{29}$ P. Laurelli, ${ }^{46}$ V. Lavorini, ${ }^{36 a, 36 b}$ W. Lavrijsen, ${ }^{14}$ P. Laycock, ${ }^{72}$ O. Le Dortz ${ }^{77}$ E. Le Guirriec,${ }^{82}$ C. Le Maner, ${ }^{157}$ E. Le Menedeu, ${ }^{11}$ T. LeCompte, ${ }^{5}$

F. Ledroit-Guillon, ${ }^{54}$ H. Lee, ${ }^{104}$ J. S. H. Lee, ${ }^{115}$ S. C. Lee, ${ }^{150}$ L. Lee, ${ }^{175}$ M. Lefebvre, ${ }^{168}$ M. Legendre,${ }^{135}$ B. C. LeGeyt, ${ }^{119}$ F. Legger, ${ }^{97}$ C. Leggett, ${ }^{14}$ M. Lehmacher, ${ }^{20}$ G. Lehmann Miotto,${ }^{29}$ X. Lei, ${ }^{6}$ M. A. L. Leite,${ }^{23 d}$ R. Leitner, ${ }^{125}$ D. Lellouch, ${ }^{171}$ B. Lemmer, ${ }^{53}$ V. Lendermann, ${ }^{57 a}$ K. J. C. Leney, ${ }^{144 b}$ T. Lenz, ${ }^{104}$ G. Lenzen, ${ }^{174}$ B. Lenzi ${ }^{29}$ K. Leonhardt, ${ }^{43}$ S. Leontsinis, ${ }^{9}$ F. Lepold, ${ }^{57 a}$ C. Leroy, ${ }^{92}$ J-R. Lessard, ${ }^{168}$ C. G. Lester, ${ }^{27}$ C. M. Lester, ${ }^{119}$ J. Levêque, ${ }^{4}$ D. Levin, ${ }^{86}$ L. J. Levinson, ${ }^{171}$ A. Lewis,${ }^{117}$ G. H. Lewis, ${ }^{107}$ A. M. Leyko, ${ }^{20}$ M. Leyton, ${ }^{15}$ B. Li,${ }^{82}$ H. Li, ${ }^{172, v}$ S. Li,${ }^{32 b, w}$ X. Li, ${ }^{86}$ Z. Liang, ${ }^{117, x}$ H. Liao, ${ }^{33}$ B. Liberti, ${ }^{132 a}$ P. Lichard,${ }^{29}$ M. Lichtnecker,${ }^{97}$ K. Lie, ${ }^{164}$ W. Liebig, ${ }^{13}$ C. Limbach, ${ }^{20}$ A. Limosani ${ }^{85}$ M. Limper, ${ }^{61}$ S. C. Lin, ${ }^{150, y}$ F. Linde, ${ }^{104}$ J. T. Linnemann, ${ }^{87}$ E. Lipeles, ${ }^{119}$ A. Lipniacka, ${ }^{13}$ T. M. Liss, ${ }^{164}$ D. Lissauer, ${ }^{24}$ A. Lister, ${ }^{48}$ A. M. Litke, ${ }^{136}$ C. Liu, ${ }^{28}$ D. Liu, ${ }^{150}$ H. Liu, ${ }^{86}$ J. B. Liu, ${ }^{86}$ M. Liu, ${ }^{32 b}$ Y. Liu, ${ }^{32 b}$ M. Livan, ${ }^{118 a, 118 b}$ S. S. A. Livermore, ${ }^{117}$ A. Lleres, ${ }^{54}$ J. Llorente Merino, ${ }^{79}$ S. L. Lloyd,${ }^{74}$ E. Lobodzinska, ${ }^{41}$ P. Loch,${ }^{6}$ W. S. Lockman, ${ }^{136}$ T. Loddenkoetter ${ }^{20}$ F. K. Loebinger, ${ }^{81}$ A. Loginov, ${ }^{175}$ C. W. Loh, ${ }^{167}$ T. Lohse,${ }^{15}$ K. Lohwasser, ${ }^{47}$ M. Lokajicek, ${ }^{124}$ V. P. Lombardo,${ }^{4}$ R. E. Long,${ }^{70}$ L. Lopes, ${ }^{123 a}$ D. Lopez Mateos, ${ }^{56}$ J. Lorenz, ${ }^{97}$ N. Lorenzo Martinez, ${ }^{114}$ M. Losada, ${ }^{161}$ P. Loscutoff, ${ }^{14}$ F. Lo Sterzo, ${ }^{131 a, 131 b}$ M. J. Losty, ${ }^{158 a}$ X. Lou, ${ }^{40}$ A. Lounis, ${ }^{114}$ K. F. Loureiro, ${ }^{161}$ J. Love,${ }^{21}$ P. A. Love, ${ }^{70}$ A. J. Lowe,${ }^{142, f}$ F. Lu, ${ }^{32 a}$ H. J. Lubatti, ${ }^{137}$ C. Luci, ${ }^{131 a, 131 b}$ A. Lucotte, ${ }^{54}$ A. Ludwig, ${ }^{43}$ D. Ludwig, ${ }^{41}$ I. Ludwig, ${ }^{47}$ J. Ludwig, ${ }^{47}$ F. Luehring, ${ }^{59}$ G. Luijckx, ${ }^{104}$ W. Lukas, ${ }^{60}$ D. Lumb,${ }^{47}$ L. Luminari, ${ }^{131 a}$ E. Lund, ${ }^{116}$ B. Lund-Jensen, ${ }^{146}$ B. Lundberg, ${ }^{78}$ J. Lundberg, ${ }^{145 a, 145 b}$ O. Lundberg, ${ }^{145 a, 145 b}$ J. Lundquist,${ }^{35}$ M. Lungwitz,${ }^{80}$ D. Lynn, ${ }^{24}$ E. Lytken, ${ }^{78}$ H. Ma, ${ }^{24}$ L. L. Ma, ${ }^{172}$ J. A. Macana Goia, ${ }^{92}$ G. Maccarrone,${ }^{46}$ A. Macchiolo, ${ }^{98}$ B. Maček, ${ }^{73}$ J. Machado Miguens, ${ }^{123 a}$ R. Mackeprang, ${ }^{35}$ R. J. Madaras, ${ }^{14}$ W. F. Mader, ${ }^{43}$ R. Maenner ${ }^{57 \mathrm{c}}$ T. Maeno, ${ }^{24}$ P. Mättig, ${ }^{174}$ S. Mättig, ${ }^{41}$ L. Magnoni, ${ }^{29}$ E. Magradze, ${ }^{53}$ K. Mahboubi,${ }^{47}$ S. Mahmoud ${ }^{72}$ G. Mahout,${ }^{17}$ C. Maiani, ${ }^{135}$ C. Maidantchik ${ }^{23 a}$ A. Maio, ${ }^{123 a, c}$ S. Majewski, ${ }^{24}$ 
Y. Makida,${ }^{64}$ N. Makovec, ${ }^{114}$ P. Mal, ${ }^{135}$ B. Malaescu, ${ }^{29}$ Pa. Malecki, ${ }^{38}$ P. Malecki, ${ }^{38}$ V. P. Maleev, ${ }^{120}$ F. Malek,${ }^{54}$ U. Mallik,${ }^{61}$ D. Malon, ${ }^{5}$ C. Malone,${ }^{142}$ S. Maltezos, ${ }^{9}$ V. Malyshev,${ }^{106}$ S. Malyukov,${ }^{29}$ R. Mameghani, ${ }^{97}$ J. Mamuzic, ${ }^{12 b}$ A. Manabe ${ }^{64}$ L. Mandelli, ${ }^{88 a}$ I. Mandić ${ }^{73}$ R. Mandrysch, ${ }^{15}$ J. Maneira, ${ }^{123 a}$ P. S. Mangeard, ${ }^{87}$ L. Manhaes de Andrade Filho, ${ }^{23 a}$ A. Mann, ${ }^{53}$ P. M. Manning, ${ }^{136}$ A. Manousakis-Katsikakis, ${ }^{8}$ B. Mansoulie, ${ }^{135}$ A. Mapelli, ${ }^{29}$ L. Mapelli, ${ }^{29}$ L. March, ${ }^{79}$ J. F. Marchand, ${ }^{28}$ F. Marchese, ${ }^{132 a, 132 b}$ G. Marchiori, ${ }^{77}$ M. Marcisovsky, ${ }^{124}$ C. P. Marino, ${ }^{168}$ F. Marroquim, ${ }^{23 a}$ Z. Marshall, ${ }^{29}$ F. K. Martens, ${ }^{157}$ S. Marti-Garcia, ${ }^{166}$ B. Martin, ${ }^{29}$ B. Martin, ${ }^{87}$

J. P. Martin, ${ }^{92}$ T. A. Martin, ${ }^{17}$ V. J. Martin, ${ }^{45}$ B. Martin dit Latour, ${ }^{48}$ S. Martin-Haugh, ${ }^{148}$ M. Martinez, ${ }^{11}$

V. Martinez Outschoorn, ${ }^{56}$ A. C. Martyniuk, ${ }^{168}$ M. Marx,${ }^{81}$ F. Marzano, ${ }^{131 a}$ A. Marzin,${ }^{110}$ L. Masetti, ${ }^{80}$ T. Mashimo, ${ }^{154}$ R. Mashinistov, ${ }^{93}$ J. Masik, ${ }^{81}$ A. L. Maslennikov, ${ }^{106}$ I. Massa, ${ }^{19 a, 19 b}$ G. Massaro, ${ }^{104}$ N. Massol, ${ }^{4}$ A. Mastroberardino, ${ }^{36 a, 36 b}$ T. Masubuchi, ${ }^{154}$ P. Matricon, ${ }^{114}$ H. Matsunaga,${ }^{154}$ T. Matsushita, ${ }^{65}$ C. Mattravers, ${ }^{117, d}$ J. Maurer, ${ }^{82}$ S. J. Maxfield, ${ }^{72}$ A. Mayne, ${ }^{138}$ R. Mazini, ${ }^{150}$ M. Mazur, ${ }^{20}$ L. Mazzaferro, ${ }^{132 a, 132 b}$ M. Mazzanti, ${ }^{88 a}$ S. P. Mc Kee ${ }^{86}$ A. McCarn, ${ }^{164}$ R. L. McCarthy, ${ }^{147}$ T. G. McCarthy,${ }^{28}$ N. A. McCubbin, ${ }^{128}$ K. W. McFarlane, ${ }^{55, a}$ J. A. Mcfayden, ${ }^{138}$ H. McGlone, ${ }^{52}$ G. Mchedlidze ${ }^{50 b}$ T. Mclaughlan, ${ }^{17}$ S. J. McMahon, ${ }^{128}$ R. A. McPherson, ${ }^{168,1}$ A. Meade ${ }^{83}$ J. Mechnich, ${ }^{104}$ M. Mechtel,,${ }^{174}$ M. Medinnis, ${ }^{41}$ R. Meera-Lebbai, ${ }^{110}$ T. Meguro, ${ }^{115}$ R. Mehdiyev ${ }^{92}$ S. Mehlhase, ${ }^{35}$ A. Mehta, ${ }^{72}$ K. Meier,${ }^{57 a}$ B. Meirose,${ }^{78}$ C. Melachrinos, ${ }^{30}$ B. R. Mellado Garcia,${ }^{172}$ F. Meloni, ${ }^{88 a, 88 b}$ L. Mendoza Navas, ${ }^{161}$ Z. Meng, ${ }^{150, v}$ A. Mengarelli ${ }^{19 a, 19 b}$ S. Menke, ${ }^{98}$ E. Meoni, ${ }^{160}$ K. M. Mercurio, ${ }^{56}$ P. Mermod, ${ }^{48}$ L. Merola, ${ }^{101 \mathrm{a}, 101 \mathrm{~b}}$ C. Meroni, ${ }^{88 \mathrm{a}}$ F. S. Merritt, ${ }^{30}$ H. Merritt, ${ }^{108}$ A. Messina,${ }^{29, \mathrm{z}}$ J. Metcalfe, ${ }^{102}$ A. S. Mete, ${ }^{162}$ C. Meyer, ${ }^{80}$ C. Meyer,${ }^{30}$ J-P. Meyer, ${ }^{135}$ J. Meyer,${ }^{173}$ J. Meyer, ${ }^{53}$ T. C. Meyer ${ }^{29}$ W. T. Meyer, ${ }^{62}$ J. Miao, ${ }^{32 \mathrm{~d}}$ S. Michal, ${ }^{29}$ L. Micu, ${ }^{25 a}$ R. P. Middleton, ${ }^{128}$ S. Migas, ${ }^{72}$ L. Mijović, ${ }^{135}$ G. Mikenberg, ${ }^{171}$ M. Mikestikova, ${ }^{124}$ M. Mikuž ${ }^{73}$ D. W. Miller, ${ }^{30}$ R. J. Miller, ${ }^{87}$ W. J. Mills, ${ }^{167}$ C. Mills,${ }^{56}$ A. Milov, ${ }^{171}$ D. A. Milstead,${ }^{145 a, 145 b}$ D. Milstein, ${ }^{171}$ A. A. Minaenko, ${ }^{127}$ M. Miñano Moya, ${ }^{166}$ I. A. Minashvili, ${ }^{63}$ A. I. Mincer, ${ }^{107}$ B. Mindur, ${ }^{37}$ M. Mineev, ${ }^{63}$ Y. Ming, ${ }^{172}$ L. M. Mir, ${ }^{11}$ G. Mirabelli, ${ }^{131 a}$ J. Mitrevski, ${ }^{136}$ V. A. Mitsou, ${ }^{166}$ S. Mitsui, ${ }^{64}$ P. S. Miyagawa, ${ }^{138}$ K. Miyazaki, ${ }^{65}$ J. U. Mjörnmark, ${ }^{78}$ T. Moa, ${ }^{145 a, 145 b}$ V. Moeller, ${ }^{27}$ K. Mönig, ${ }^{41}$ N. Möser ${ }^{20}$ S. Mohapatra, ${ }^{147}$ W. Mohr,${ }^{47}$ R. Moles-Valls, ${ }^{166}$ J. Molina-Perez,${ }^{29}$ J. Monk, ${ }^{76}$ E. Monnier ${ }^{82}$ S. Montesano,${ }^{88 a, 88 b}$ F. Monticelli, ${ }^{69}$ S. Monzani, ${ }^{19 a, 19 b}$ R. W. Moore, ${ }^{2}$ G. F. Moorhead ${ }^{85}$ C. Mora Herrera ${ }^{48}$ A. Moraes ${ }^{52}$ N. Morange, ${ }^{135}$ J. Morel,${ }^{53}$ G. Morello, ${ }^{36 a, 36 b}$ D. Moreno, ${ }^{80}$ M. Moreno Llácer, ${ }^{166}$ P. Morettini, ${ }^{49 a}$ M. Morgenstern,${ }^{43}$ M. Morii, ${ }^{56}$ A. K. Morley, ${ }^{29}$ G. Mornacchi, ${ }^{29}$ J. D. Morris,${ }^{74}$ L. Morvaj, ${ }^{100}$ H. G. Moser, ${ }^{98}$ M. Mosidze, ${ }^{50 b}$ J. Moss, ${ }^{108}$ R. Mount, ${ }^{142}$ E. Mountricha, ${ }^{9, a a}$ S. V. Mouraviev, ${ }^{93, a}$ E. J. W. Moyse, ${ }^{83}$ F. Mueller, ${ }^{57 a}$ J. Mueller, ${ }^{122}$ K. Mueller ${ }^{20}$ T. A. Müller, ${ }^{97}$ T. Mueller, ${ }^{80}$ D. Muenstermann, ${ }^{29}$ Y. Munwes, ${ }^{152}$ W. J. Murray, ${ }^{128}$ I. Mussche, ${ }^{104}$ E. Musto, ${ }^{101 a, 101 b}$ A. G. Myagkov, ${ }^{127}$ M. Myska, ${ }^{124}$ J. Nadal,${ }^{11}$ K. Nagai,${ }^{159}$ K. Nagano ${ }^{64}$ A. Nagarkar,${ }^{108}$ Y. Nagasaka, ${ }^{58}$ M. Nagel,${ }^{98}$

A. M. Nairz, ${ }^{29}$ Y. Nakahama, ${ }^{29}$ K. Nakamura, ${ }^{154}$ T. Nakamura, ${ }^{154}$ I. Nakano, ${ }^{109}$ G. Nanava, ${ }^{20}$ A. Napier, ${ }^{160}$ R. Narayan, ${ }^{57 b}$ M. Nash, ${ }^{76, d}$ T. Nattermann,${ }^{20}$ T. Naumann, ${ }^{41}$ G. Navarro, ${ }^{161}$ H. A. Neal, ${ }^{86}$ P. Yu. Nechaeva,${ }^{93}$ T. J. Neep, ${ }^{81}$ A. Negri, ${ }^{118 a, 118 b}$ G. Negri, ${ }^{29}$ S. Nektarijevic, ${ }^{48}$ A. Nelson, ${ }^{162}$ T. K. Nelson, ${ }^{142}$ S. Nemecek, ${ }^{124}$ P. Nemethy, ${ }^{107}$ A. A. Nepomuceno, ${ }^{23 a}$ M. Nessi, ${ }^{29, b b}$ M. S. Neubauer,${ }^{164}$ A. Neusiedl, ${ }^{80}$ R. M. Neves, ${ }^{107}$ P. Nevski, ${ }^{24}$ P. R. Newman, ${ }^{17}$ V. Nguyen Thi Hong, ${ }^{135}$ R. B. Nickerson, ${ }^{117}$ R. Nicolaidou, ${ }^{135}$ B. Nicquevert, ${ }^{29}$ F. Niedercorn, ${ }^{114}$ J. Nielsen, ${ }^{136}$ N. Nikiforou, ${ }^{34}$ A. Nikiforov, ${ }^{15}$ V. Nikolaenko, ${ }^{127}$ I. Nikolic-Audit, ${ }^{77}$ K. Nikolics,${ }^{48}$ K. Nikolopoulos, ${ }^{24}$ H. Nilsen, ${ }^{47}$ P. Nilsson, ${ }^{7}$ Y. Ninomiya, ${ }^{154}$ A. Nisati, ${ }^{131 a}$ T. Nishiyama,${ }^{65}$ R. Nisius, ${ }^{98}$ L. Nodulman, ${ }^{5}$ M. Nomachi,${ }^{115}$ I. Nomidis, ${ }^{153}$ M. Nordberg, ${ }^{29}$ P. R. Norton, ${ }^{128}$ J. Novakova, ${ }^{125}$ M. Nozaki, ${ }^{64}$ L. Nozka, ${ }^{112}$ I. M. Nugent, ${ }^{158 a}$ A.-E. Nuncio-Quiroz, ${ }^{20}$ G. Nunes Hanninger, ${ }^{85}$ T. Nunnemann, ${ }^{97}$ E. Nurse,${ }^{76}$ B. J. O'Brien ${ }^{45}$ S. W. O’Neale, ${ }^{17, a}$ D. C. O'Neill, ${ }^{141}$ V. O'Shea, ${ }^{52}$ L. B. Oakes, ${ }^{97}$ F. G. Oakham, ${ }^{28, e}$ H. Oberlack, ${ }^{98}$ J. Ocariz, ${ }^{77}$ A. Ochi, ${ }^{65}$ S. Oda, ${ }^{68}$ S. Odaka, ${ }^{64}$ J. Odier,${ }^{82}$ H. Ogren ${ }^{59}$ A. Oh,${ }^{81}$ S. H. Oh,${ }^{44}$ C. C. Ohm,${ }^{145 a, 145 b}$ T. Ohshima, ${ }^{100}$ S. Okada, ${ }^{65}$ H. Okawa, ${ }^{162}$ Y. Okumura, ${ }^{100}$ T. Okuyama, ${ }^{154}$ A. Olariu, ${ }^{25 a}$ A. G. Olchevski, ${ }^{63}$ S. A. Olivares Pino, ${ }^{31 a}$ M. Oliveira, ${ }^{123 a, i}$

D. Oliveira Damazio, ${ }^{24}$ E. Oliver Garcia, ${ }^{166}$ D. Olivito, ${ }^{119}$ A. Olszewski,${ }^{38}$ J. Olszowska, ${ }^{38}$ A. Onofre, ${ }^{123 a, c c}$ P. U.E. Onyisi, ${ }^{30}$ C. J. Oram, ${ }^{158 a}$ M. J. Oreglia, ${ }^{30}$ Y. Oren, ${ }^{152}$ D. Orestano, ${ }^{133 a, 133 b}$ N. Orlando, ${ }^{71 a, 71 b}$ I. Orlov, ${ }^{106}$ C. Oropeza Barrera, ${ }^{52}$ R. S. Orr, ${ }^{157}$ B. Osculati, ${ }^{49 a, 49 b}$ R. Ospanov, ${ }^{119}$ C. Osuna, ${ }^{11}$ G. Otero y Garzon, ${ }^{26}$ J. P. Ottersbach, ${ }^{104}$ M. Ouchrif, ${ }^{134 \mathrm{~d}}$ E. A. Ouellette, ${ }^{168}$ F. Ould-Saada, ${ }^{116}$ A. Ouraou, ${ }^{135}$ Q. Ouyang, ${ }^{32 a}$ A. Ovcharova, ${ }^{14}$ M. Owen, ${ }^{81}$ S. Owen, ${ }^{138}$ V. E. Ozcan, ${ }^{18 a}$ N. Ozturk, ${ }^{7}$ A. Pacheco Pages, ${ }^{11}$ C. Padilla Aranda, ${ }^{11}$ S. Pagan Griso, ${ }^{14}$ E. Paganis, ${ }^{138}$ F. Paige,${ }^{24}$ P. Pais,${ }^{83}$ K. Pajchel, ${ }^{116}$ G. Palacino, ${ }^{158 b}$ C. P. Paleari, ${ }^{6}$ S. Palestini, ${ }^{29}$

D. Pallin, ${ }^{33}$ A. Palma, ${ }^{123 a}$ J. D. Palmer, ${ }^{17}$ Y. B. Pan, ${ }^{172}$ E. Panagiotopoulou, ${ }^{9}$ P. Pani, ${ }^{104}$ N. Panikashvili,${ }^{86}$ S. Panitkin, ${ }^{24}$ D. Pantea, ${ }^{25 a}$ A. Papadelis, ${ }^{145 a}$ Th. D. Papadopoulou, ${ }^{9}$ A. Paramonov, ${ }^{5}$ D. Paredes Hernandez, ${ }^{33}$ W. Park, ${ }^{24, d d}$ M. A. Parker,${ }^{27}$ F. Parodi, ${ }^{49 a, 49 b}$ J. A. Parsons,${ }^{34}$ U. Parzefall,${ }^{47}$ S. Pashapour, ${ }^{53}$ E. Pasqualucci, ${ }^{131 a}$ 
S. Passaggio, ${ }^{49 a}$ A. Passeri, ${ }^{133 a}$ F. Pastore, ${ }^{133 a, 133 b, a}$ Fr. Pastore, ${ }^{75}$ G. Pásztor, ${ }^{48, e e}$ S. Pataraia, ${ }^{174}$ N. Patel,,${ }^{149}$ J. R. Pater, ${ }^{81}$ S. Patricelli, ${ }^{101 \mathrm{a}, 101 \mathrm{~b}}$ T. Pauly, ${ }^{29}$ M. Pecsy,${ }^{143 \mathrm{a}}$ M. I. Pedraza Morales, ${ }^{172}$ S. V. Peleganchuk, ${ }^{106}$ D. Pelikan, ${ }^{165}$ H. Peng, ${ }^{32 b}$ B. Penning, ${ }^{30}$ A. Penson, ${ }^{34}$ J. Penwell,${ }^{59}$ M. Perantoni,${ }^{23 a}$ K. Perez,${ }^{34, f f}$

T. Perez Cavalcanti, ${ }^{41}$ E. Perez Codina, ${ }^{158 a}$ M. T. Pérez García-Estañ, ${ }^{166}$ V. Perez Reale,${ }^{34}$ L. Perini, ${ }^{88 a, 88 b}$ H. Pernegger, ${ }^{29}$ R. Perrino, ${ }^{71 a}$ P. Perrodo, ${ }^{4}$ V. D. Peshekhonov, ${ }^{63}$ K. Peters,${ }^{29}$ B. A. Petersen, ${ }^{29}$ J. Petersen,${ }^{29}$ T. C. Petersen, ${ }^{35}$ E. Petit, ${ }^{4}$ A. Petridis, ${ }^{153}$ C. Petridou, ${ }^{153}$ E. Petrolo, ${ }^{131 a}$ F. Petrucci, ${ }^{133 a, 133 b}$ D. Petschull, ${ }^{41}$ M. Petteni, ${ }^{141}$ R. Pezoa ${ }^{31 b}$ A. Phan,${ }^{85}$ P. W. Phillips, ${ }^{128}$ G. Piacquadio, ${ }^{29}$ A. Picazio, ${ }^{48}$ E. Piccaro, ${ }^{74}$ M. Piccinini, ${ }^{19 a, 19 b}$ S. M. Piec, ${ }^{41}$ R. Piegaia,${ }^{26}$ D. T. Pignotti ${ }^{108}$ J. E. Pilcher, ${ }^{30}$ A. D. Pilkington, ${ }^{81}$ J. Pina, ${ }^{123 a, c}$ M. Pinamonti, ${ }^{163 a, 163 c}$ A. Pinder, ${ }^{117}$ J. L. Pinfold,${ }^{2}$ B. Pinto, ${ }^{123 a}$ C. Pizio,${ }^{88 a, 88 b}$ M. Plamondon, ${ }^{168}$ M.-A. Pleier,${ }^{24}$ E. Plotnikova, ${ }^{63}$ A. Poblaguev, ${ }^{24}$ S. Poddar, ${ }^{57 a}$ F. Podlyski, ${ }^{33}$ L. Poggioli, ${ }^{114}$ T. Poghosyan, ${ }^{20}$ M. Pohl,${ }^{48}$ F. Polci, ${ }^{54}$ G. Polesello, ${ }^{18 \mathrm{a}}$ A. Policicchio, ${ }^{36 \mathrm{a}, 36 \mathrm{~b}}$ A. Polini, ${ }^{19 \mathrm{a}} \mathrm{J}$. Poll,${ }^{74}$ V. Polychronakos,${ }^{24}$ D. Pomeroy,${ }^{22} \mathrm{~K}$. Pommès, ${ }^{29}$

L. Pontecorvo, ${ }^{131 a}$ B. G. Pope,${ }^{87}$ G. A. Popeneciu, ${ }^{25 a}$ D. S. Popovic, ${ }^{12 a}$ A. Poppleton, ${ }^{29}$ X. Portell Bueso,${ }^{29}$

G. E. Pospelov, ${ }^{98}$ S. Pospisil,${ }^{126}$ I. N. Potrap,${ }^{98}$ C. J. Potter, ${ }^{148}$ C. T. Potter, ${ }^{113}$ G. Poulard, ${ }^{29}$ J. Poveda,${ }^{59}$ V. Pozdnyakov, ${ }^{63}$ R. Prabhu ${ }^{76}$ P. Pralavorio, ${ }^{82}$ A. Pranko, ${ }^{14}$ S. Prasad,${ }^{29}$ R. Pravahan, ${ }^{24}$ S. Prell, ${ }^{62}$ K. Pretzl, ${ }^{16}$ D. Price, ${ }^{59}$ J. Price, ${ }^{72}$ L. E. Price,${ }^{5}$ D. Prieur, ${ }^{122}$ M. Primavera, ${ }^{71 a}$ K. Prokofiev, ${ }^{107}$ F. Prokoshin,${ }^{31 b}$ S. Protopopescu, ${ }^{24}$

J. Proudfoot, ${ }^{5}$ X. Prudent,${ }^{43}$ M. Przybycien, ${ }^{37}$ H. Przysiezniak, ${ }^{4}$ S. Psoroulas, ${ }^{20}$ E. Ptacek, ${ }^{113}$ E. Pueschel,${ }^{83}$ J. Purdham, ${ }^{86}$ M. Purohit, ${ }^{24, d d}$ P. Puzo, ${ }^{114}$ Y. Pylypchenko, ${ }^{61}$ J. Qian,${ }^{86}$ A. Quadt, ${ }^{53}$ D. R. Quarrie, ${ }^{14}$ W. B. Quayle, ${ }^{172}$ F. Quinonez, ${ }^{31 \mathrm{a}}$ M. Raas, ${ }^{103}$ V. Radescu, ${ }^{41}$ P. Radloff, ${ }^{113}$ T. Rador, ${ }^{18 a}$ F. Ragusa, ${ }^{88 a},{ }^{88 b}$ G. Rahal,${ }^{177}$ A. M. Rahimi, ${ }^{108}$ D. Rahm, ${ }^{24}$ S. Rajagopalan,${ }^{24}$ M. Rammensee,${ }^{47}$ M. Rammes ${ }^{140}$ A. S. Randle-Conde, ${ }^{39} \mathrm{~K}$. Randrianarivony,${ }^{28}$ F. Rauscher, ${ }^{97}$ T. C. Rave,${ }^{47}$ M. Raymond ${ }^{29}$ A. L. Read, ${ }^{116}$ D. M. Rebuzzi, ${ }^{118 a, 118 b}$ A. Redelbach, ${ }^{173}$ G. Redlinger,${ }^{24}$ R. Reece, ${ }^{119}$ K. Reeves, ${ }^{40}$ E. Reinherz-Aronis, ${ }^{152}$ A. Reinsch,${ }^{113}$ I. Reisinger, ${ }^{42}$ C. Rembser ${ }^{29}$ Z. L. Ren, ${ }^{150}$

A. Renaud, ${ }^{114}$ M. Rescigno, ${ }^{131 a}$ S. Resconi, ${ }^{88 a}$ B. Resende, ${ }^{135}$ P. Reznicek,${ }^{97}$ R. Rezvani, ${ }^{157}$ R. Richter,${ }^{98}$ E. Richter-Was, ${ }^{4, g g}$ M. Ridel, ${ }^{77}$ M. Rijpstra, ${ }^{104}$ M. Rijssenbeek, ${ }^{147}$ A. Rimoldi, ${ }^{118 a, 118 b}$ L. Rinaldi, ${ }^{19 a}$ R. R. Rios, ${ }^{39}$ I. Riu, ${ }^{11}$ G. Rivoltella, ${ }^{88 a}, 88 \mathrm{~b}$ F. Rizatdinova, ${ }^{111}$ E. Rizvi, ${ }^{74}$ S. H. Robertson, ${ }^{84,1}$ A. Robichaud-Veronneau, ${ }^{117}$ D. Robinson, ${ }^{27}$ J. E. M. Robinson, ${ }^{76}$ A. Robson, ${ }^{52}$ J. G. Rocha de Lima, ${ }^{105}$ C. Roda, ${ }^{121 a, 121 b}$ D. Roda Dos Santos, ${ }^{29}$ A. Roe, ${ }^{53}$ S. Roe, ${ }^{29}$ O. Røhne, ${ }^{116}$ S. Rolli, ${ }^{160}$ A. Romaniouk,,${ }^{95}$ M. Romano, ${ }^{19 a, 19 b}$ G. Romeo, ${ }^{26}$ E. Romero Adam, ${ }^{166}$ L. Roos, ${ }^{77}$ E. Ros, ${ }^{166}$ S. Rosati, ${ }^{131 a}$ K. Rosbach, ${ }^{48}$ A. Rose,${ }^{148}$ M. Rose, ${ }^{75}$ G. A. Rosenbaum,,${ }^{157}$ E. I. Rosenberg, ${ }^{62}$ P. L. Rosendahl, ${ }^{13}$ O. Rosenthal, ${ }^{140}$ L. Rosselet, ${ }^{48}$ V. Rossetti, ${ }^{11}$ E. Rossi, ${ }^{131 a, 131 b}$ L. P. Rossi ${ }^{49 a}$ M. Rotaru, ${ }^{25 a}$ I. Roth, ${ }^{171}$ J. Rothberg, ${ }^{137}$ D. Rousseau, ${ }^{114}$ C. R. Royon, ${ }^{135}$ A. Rozanov, ${ }^{82}$ Y. Rozen, ${ }^{151}$ X. Ruan, ${ }^{32 a, h h}$ F. Rubbo, ${ }^{11}$ I. Rubinskiy, ${ }^{41}$ B. Ruckert, ${ }^{97}$ N. Ruckstuhl, ${ }^{104}$ V. I. Rud,${ }^{96}$ C. Rudolph,${ }^{43}$ G. Rudolph, ${ }^{60}$ F. Rühr, ${ }^{6}$ F. Ruggieri, ${ }^{133 a, 133 b}$ A. Ruiz-Martinez, ${ }^{62}$ L. Rumyantsev, ${ }^{63}$ Z. Rurikova, ${ }^{47}$ N. A. Rusakovich, ${ }^{63}$ J. P. Rutherfoord, ${ }^{6}$ C. Ruwiedel, ${ }^{14, a}$ P. Ruzicka, ${ }^{124}$ Y. F. Ryabov, ${ }^{120}$ P. Ryan,${ }^{87}$ M. Rybar, ${ }^{125}$ G. Rybkin, ${ }^{114}$ N. C. Ryder, ${ }^{117}$ A. F. Saavedra, ${ }^{149}$ I. Sadeh ${ }^{152}$

H. F. -W. Sadrozinski, ${ }^{136}$ R. Sadykov, ${ }^{63}$ F. Safai Tehrani, ${ }^{131 a}$ H. Sakamoto, ${ }^{154}$ G. Salamanna, ${ }^{74}$ A. Salamon, ${ }^{132 a}$ M. Saleem, ${ }^{110}$ D. Salek, ${ }^{29}$ D. Salihagic, ${ }^{98}$ A. Salnikov, ${ }^{142}$ J. Salt, ${ }^{166}$ B. M. Salvachua Ferrando, ${ }^{5}$ D. Salvatore, ${ }^{36 a, 36 b}$ F. Salvatore, ${ }^{148}$ A. Salvucci, ${ }^{103}$ A. Salzburger, ${ }^{29}$ D. Sampsonidis, ${ }^{153}$ B. H. Samset, ${ }^{116}$ A. Sanchez, ${ }^{101 a, 101 b}$ V. Sanchez Martinez, ${ }^{166}$ H. Sandaker, ${ }^{13}$ H. G. Sander,${ }^{80}$ M. P. Sanders, ${ }^{97}$ M. Sandhoff, ${ }^{174}$ T. Sandoval, ${ }^{27}$ C. Sandoval,${ }^{161}$ R. Sandstroem,${ }^{98}$ D. P. C. Sankey, ${ }^{128}$ A. Sansoni, ${ }^{46}$ C. Santamarina Rios,${ }^{84}$ C. Santoni,${ }^{33}$ R. Santonico, ${ }^{132 a, 132 b}$ H. Santos, ${ }^{123 a}$ J. G. Saraiva, ${ }^{123 a}$ T. Sarangi, ${ }^{172}$ E. Sarkisyan-Grinbaum, ${ }^{7}$ F. Sarri, ${ }^{121 a, 121 b}$ G. Sartisohn, ${ }^{174}$ O. Sasaki, ${ }^{64}$ N. Sasao, ${ }^{66}$ I. Satsounkevitch,${ }^{89}$ G. Sauvage, ${ }^{4, a}$ E. Sauvan, ${ }^{4}$ J. B. Sauvan, ${ }^{114}$ P. Savard ${ }^{157, \mathrm{e}}$ V. Savinov, ${ }^{122}$ D. O. Savu, ${ }^{29}$ L. Sawyer, ${ }^{24, \mathrm{n}}$ D. H. Saxon, ${ }^{52}$ J. Saxon, ${ }^{119}$ C. Sbarra, ${ }^{19 a}$ A. Sbrizzi, ${ }^{19 a, 19 b}$ O. Scallon, ${ }^{92}$ D. A. Scannicchio, ${ }^{162}$ M. Scarcella, ${ }^{149}$ J. Schaarschmidt, ${ }^{114}$ P. Schacht, ${ }^{98}$ D. Schaefer, ${ }^{119}$ U. Schäfer, ${ }^{80}$ S. Schaepe, ${ }^{20}$ S. Schaetzel,${ }^{57 b}$ A. C. Schaffer, ${ }^{114}$ D. Schaile, ${ }^{97}$ R. D. Schamberger, ${ }^{147}$ A. G. Schamov, ${ }^{106}$ V. Scharf, ${ }^{57 a}$ V. A. Schegelsky, ${ }^{120}$ D. Scheirich,${ }^{86}$ M. Schernau, ${ }^{162}$ M. I. Scherzer, ${ }^{34}$ C. Schiavi, ${ }^{49 a, 49 b}$ J. Schieck, ${ }^{97}$

M. Schioppa, ${ }^{36 a, 36 b}$ S. Schlenker, ${ }^{29}$ E. Schmidt, ${ }^{47}$ K. Schmieden, ${ }^{20}$ C. Schmitt, ${ }^{80}$ S. Schmitt, ${ }^{57 b}$ M. Schmitz, ${ }^{20}$ B. Schneider, ${ }^{16}$ U. Schnoor, ${ }^{43}$ A. Schoening, ${ }^{57 b}$ A. L. S. Schorlemmer, ${ }^{53}$ M. Schott,${ }^{29}$ D. Schouten,,${ }^{158 a}$ J. Schovancova, ${ }^{124}$ M. Schram, ${ }^{84}$ C. Schroeder, ${ }^{80}$ N. Schroer, ${ }^{57 c}$ M. J. Schultens, ${ }^{20}$ J. Schultes, ${ }^{174}$

H.-C. Schultz-Coulon, ${ }^{57 a}$ H. Schulz, ${ }^{15}$ J. W. Schumacher, ${ }^{20}$ M. Schumacher, ${ }^{47}$ B. A. Schumm, ${ }^{136}$ Ph. Schune, ${ }^{135}$ C. Schwanenberger, ${ }^{81}$ A. Schwartzman, ${ }^{142}$ Ph. Schwemling, ${ }^{77}$ R. Schwienhorst, ${ }^{87}$ R. Schwierz, ${ }^{43}$ J. Schwindling, ${ }^{135}$ T. Schwindt, ${ }^{20}$ M. Schwoerer, ${ }^{4}$ G. Sciolla, ${ }^{22}$ W. G. Scott, ${ }^{128}$ J. Searcy, ${ }^{113}$ G. Sedov,${ }^{41}$ E. Sedykh, ${ }^{120}$ S. C. Seidel, ${ }^{102}$ A. Seiden, ${ }^{136}$ F. Seifert, ${ }^{43}$ J. M. Seixas, ${ }^{23 a}$ G. Sekhniaidze, ${ }^{101 a}$ S. J. Sekula ${ }^{39}$ K. E. Selbach, ${ }^{45}$ D. M. Seliverstov, ${ }^{120}$ B. Sellden, ${ }^{145 a}$ G. Sellers, ${ }^{72}$ M. Seman, ${ }^{143 b}$ N. Semprini-Cesari, ${ }^{19 a, 19 b}$ C. Serfon,${ }^{97}$ L. Serin, ${ }^{114}$ L. Serkin,${ }^{53}$ 
R. Seuster, ${ }^{98}$ H. Severini, ${ }^{110}$ A. Sfyrla, ${ }^{29}$ E. Shabalina, ${ }^{53}$ M. Shamim, ${ }^{113}$ L. Y. Shan, ${ }^{32 a}$ J. T. Shank, ${ }^{21}$ Q. T. Shao, ${ }^{85}$ M. Shapiro, ${ }^{14}$ P. B. Shatalov, ${ }^{94}$ K. Shaw, ${ }^{163 a, 163 c}$ D. Sherman, ${ }^{175}$ P. Sherwood ${ }^{76}$ A. Shibata, ${ }^{107}$ H. Shichi, ${ }^{100}$ S. Shimizu, ${ }^{29}$ M. Shimojima, ${ }^{99}$ T. Shin,${ }^{55}$ M. Shiyakova, ${ }^{63}$ A. Shmeleva, ${ }^{93}$ M. J. Shochet, ${ }^{30}$ D. Short, ${ }^{117}$ S. Shrestha, ${ }^{62}$ E. Shulga, ${ }^{95}$ M. A. Shupe, ${ }^{6}$ P. Sicho, ${ }^{124}$ A. Sidoti, ${ }^{131 a}$ F. Siegert, ${ }^{47}$ Dj. Sijacki, ${ }^{12 a}$ O. Silbert, ${ }^{171}$ J. Silva, ${ }^{123 a}$ Y. Silver, ${ }^{152}$ D. Silverstein, ${ }^{142}$ S. B. Silverstein, ${ }^{145 a}$ V. Simak, ${ }^{126}$ O. Simard, ${ }^{135}$ Lj. Simic, ${ }^{12 a}$ S. Simion, ${ }^{114}$ E. Simioni, ${ }^{80}$ B. Simmons, ${ }^{76}$ R. Simoniello, ${ }^{88 a, 88 b}$ M. Simonyan, ${ }^{35}$ P. Sinervo, ${ }^{157}$ N. B. Sinev, ${ }^{113}$ V. Sipica, ${ }^{140}$

G. Siragusa, ${ }^{173}$ A. Sircar, ${ }^{24}$ A. N. Sisakyan, ${ }^{63, a}$ S. Yu. Sivoklokov, ${ }^{96}$ J. Sjölin,${ }^{145 a, 145 b}$ T. B. Sjursen, ${ }^{13}$

L. A. Skinnari, ${ }^{14}$ H. P. Skottowe, ${ }^{56}$ K. Skovpen, ${ }^{106}$ P. Skubic, ${ }^{110}$ M. Slater, ${ }^{17}$ T. Slavicek, ${ }^{126}$ K. Sliwa, ${ }^{160}$ V. Smakhtin, ${ }^{171}$ B. H. Smart, ${ }^{45}$ S. Yu. Smirnov, ${ }^{95}$ Y. Smirnov, ${ }^{95}$ L. N. Smirnova, ${ }^{96}$ O. Smirnova,${ }^{78}$ B. C. Smith, ${ }^{56}$ D. Smith, ${ }^{142}$ K. M. Smith, ${ }^{52}$ M. Smizanska, ${ }^{70}$ K. Smolek, ${ }^{126}$ A. A. Snesarev, ${ }^{93}$ S. W. Snow, ${ }^{81}$ J. Snow, ${ }^{110}$ S. Snyder,${ }^{24}$ R. Sobie, ${ }^{168,1}$ J. Sodomka, ${ }^{126}$ A. Soffer, ${ }^{152}$ C. A. Solans, ${ }^{166}$ M. Solar, ${ }^{126}$ J. Solc, ${ }^{126}$ E. Yu. Soldatov,${ }^{95}$ U. Soldevila, ${ }^{166}$ E. Solfaroli Camillocci, ${ }^{131 \mathrm{a}, 131 \mathrm{~b}}$ A. A. Solodkov, ${ }^{127}$ O. V. Solovyanov, ${ }^{127}$ N. Soni, ${ }^{2}$ V. Sopko, ${ }^{126}$ B. Sopko, ${ }^{126}$ M. Sosebee, ${ }^{7}$ R. Soualah, ${ }^{163 a, 163 c}$ A. Soukharev, ${ }^{106}$ S. Spagnolo, ${ }^{71 a, 71 b}$ F. Spanò,${ }^{75}$ R. Spighi, ${ }^{19 a}$ G. Spigo, ${ }^{29}$ F. Spila, ${ }^{131 \mathrm{a}, 131 \mathrm{~b}}$ R. Spiwoks, ${ }^{29}$ M. Spousta, ${ }^{125, \text { ii }}$ T. Spreitzer, ${ }^{157}$ B. Spurlock, ${ }^{7}$ R. D. St. Denis, ${ }^{52}$ J. Stahlman, ${ }^{119}$ R. Stamen, ${ }^{57 a}$ E. Stanecka, ${ }^{38}$ R. W. Stanek, ${ }^{5}$ C. Stanescu, ${ }^{133 a}$ M. Stanescu-Bellu, ${ }^{41}$ S. Stapnes, ${ }^{116}$ E. A. Starchenko, ${ }^{127}$ J. Stark, ${ }^{54}$ P. Staroba, ${ }^{124}$ P. Starovoitov, ${ }^{41}$ A. Staude,,${ }^{97}$ P. Stavina, ${ }^{143 a, a}$ G. Steele, ${ }^{52}$ P. Steinbach, ${ }^{43}$ P. Steinberg, ${ }^{24}$ I. Stekl, ${ }^{126}$ B. Stelzer, ${ }^{141}$ H. J. Stelzer, ${ }^{87}$ O. Stelzer-Chilton, ${ }^{158 a}$ H. Stenzel, ${ }^{51}$ S. Stern, ${ }^{98}$ G. A. Stewart, ${ }^{29}$ J. A. Stillings, ${ }^{20}$ M. C. Stockton, ${ }^{84}$ K. Stoerig, ${ }^{47}$ G. Stoicea, ${ }^{25 a}$ S. Stonjek, ${ }^{98}$ P. Strachota, ${ }^{125}$ A. R. Stradling, ${ }^{7}$ A. Straessner, ${ }^{43}$ J. Strandberg, ${ }^{146}$ S. Strandberg, ${ }^{145 a, 145 b}$ A. Strandlie, ${ }^{116}$ M. Strang, ${ }^{108}$ E. Strauss, ${ }^{142}$ M. Strauss, ${ }^{110}$ P. Strizenec, ${ }^{143 b}$ R. Ströhmer, ${ }^{173}$ D. M. Strom, ${ }^{113}$ J. A. Strong, ${ }^{75, a}$ R. Stroynowski, ${ }^{39}$ J. Strube, ${ }^{128}$ B. Stugu, ${ }^{13}$ I. Stumer, ${ }^{24, a}$ J. Stupak, ${ }^{147}$ P. Sturm, ${ }^{174}$ N. A. Styles, ${ }^{41}$ D. A. Soh,${ }^{150, x}$ D. Su, ${ }^{142}$ HS. Subramania, ${ }^{2}$ A. Succurro, ${ }^{11}$ Y. Sugaya,${ }^{15}$ C. Suhr, ${ }^{105}$ K. Suita, ${ }^{65}$ M. Suk,${ }^{125}$ V. V. Sulin, ${ }^{93}$ S. Sultansoy, ${ }^{3 \mathrm{~d}}$ T. Sumida, ${ }^{66}$ X. Sun, ${ }^{54}$ J. E. Sundermann, ${ }^{47}$ K. Suruliz, ${ }^{138}$ G. Susinno, ${ }^{36 a, 36 b}$ M. R. Sutton, ${ }^{148}$ Y. Suzuki, ${ }^{64}$ Y. Suzuki, ${ }^{65}$ M. Svatos, ${ }^{124}$ S. Swedish, ${ }^{167}$ I. Sykora, ${ }^{143 a}$ T. Sykora, ${ }^{125}$ J. Sánchez, ${ }^{166}$ D. Ta,${ }^{104}$ K. Tackmann, ${ }^{41}$ A. Taffard, ${ }^{162}$ R. Tafirout, ${ }^{158 a}$ N. Taiblum, ${ }^{152}$ Y. Takahashi,${ }^{100}$ H. Takai, ${ }^{24}$ R. Takashima,${ }^{67}$ H. Takeda, ${ }^{65}$ T. Takeshita, ${ }^{139}$ Y. Takubo, ${ }^{64}$ M. Talby, ${ }^{82}$ A. Talyshev, ${ }^{106,9}$ M. C. Tamsett, ${ }^{24}$ J. Tanaka, ${ }^{154}$ R. Tanaka, ${ }^{114}$ S. Tanaka, ${ }^{130}$ S. Tanaka ${ }^{64}$ A. J. Tanasijczuk,${ }^{141}$ K. Tani, ${ }^{65}$ N. Tannoury, ${ }^{82}$ S. Tapprogge, ${ }^{80}$ D. Tardif,,${ }^{157}$ S. Tarem,${ }^{151}$ F. Tarrade, ${ }^{28}$ G. F. Tartarelli, ${ }^{88 \mathrm{a}}$ P. Tas, ${ }^{125}$ M. Tasevsky, ${ }^{124}$ E. Tassi, ${ }^{36 a, 36 \mathrm{~b}}$ M. Tatarkhanov, ${ }^{14}$ Y. Tayalati, ${ }^{134 \mathrm{~d}}$

C. Taylor, ${ }^{76}$ F. E. Taylor, ${ }^{91}$ G. N. Taylor, ${ }^{85}$ W. Taylor, ${ }^{158 b}$ M. Teinturier,${ }^{114}$ M. Teixeira Dias Castanheira,${ }^{74}$ P. Teixeira-Dias, ${ }^{75}$ K. K. Temming,${ }^{47}$ H. Ten Kate, ${ }^{29}$ P. K. Teng, ${ }^{150}$ S. Terada,${ }^{64}$ K. Terashi,,${ }^{154}$ J. Terron, ${ }^{79}$ M. Testa, ${ }^{46}$ R. J. Teuscher, ${ }^{157,1}$ J. Therhaag, ${ }^{20}$ T. Theveneaux-Pelzer, ${ }^{77}$ S. Thoma, ${ }^{47}$ J.P. Thomas, ${ }^{17}$ E. N. Thompson, ${ }^{34}$ P. D. Thompson, ${ }^{17}$ P. D. Thompson, ${ }^{157}$ A. S. Thompson, ${ }^{52}$ L. A. Thomsen, ${ }^{35}$ E. Thomson, ${ }^{119}$ M. Thomson, ${ }^{27}$ R. P. Thun, ${ }^{86}$ F. Tian, ${ }^{34}$ M. J. Tibbetts, ${ }^{14}$ T. Tic,${ }^{124}$ V. O. Tikhomirov, ${ }^{93}$ Y. A. Tikhonov, ${ }^{106,9}$ S. Timoshenko, ${ }^{95}$ P. Tipton, ${ }^{175}$ F. J. Tique Aires Viegas, ${ }^{29}$ S. Tisserant, ${ }^{82}$ T. Todorov ${ }^{4}$ S. Todorova-Nova, ${ }^{160}$ B. Toggerson, ${ }^{162}$ J. Tojo, ${ }^{68}$ S. Tokár, ${ }^{143 a} \mathrm{~K}$. Tokunaga, ${ }^{65} \mathrm{~K}$. Tokushuku, ${ }^{64} \mathrm{~K}$. Tollefson, ${ }^{87} \mathrm{M}$. Tomoto, ${ }^{100} \mathrm{~L}$. Tompkins,${ }^{30} \mathrm{~K}$. Toms,${ }^{102}$ A. Tonoyan, ${ }^{13}$ C. Topfel, ${ }^{16}$ N. D. Topilin,${ }^{63}$ I. Torchiani, ${ }^{29}$ E. Torrence, ${ }^{113}$ H. Torres,${ }^{77}$ E. Torró Pastor,,${ }^{166}$ J. Toth,,${ }^{82, e e}$ F. Touchard, ${ }^{82}$ D. R. Tovey, ${ }^{138}$ T. Trefzger, ${ }^{173}$ L. Tremblet, ${ }^{29}$ A. Tricoli, ${ }^{29}$ I. M. Trigger, ${ }^{158 a}$ S. Trincaz-Duvoid, ${ }^{77}$ M. F. Tripiana, ${ }^{69}$ W. Trischuk, ${ }^{157}$ B. Trocmé,${ }^{54} \mathrm{C}$. Troncon, ${ }^{88 a}$ M. Trottier-McDonald, ${ }^{141}$ M. Trzebinski, ${ }^{38}$ A. Trzupek, ${ }^{38}$ C. Tsarouchas, ${ }^{29}$ J. C. -L. Tseng, ${ }^{117}$ M. Tsiakiris, ${ }^{104}$ P. V. Tsiareshka, ${ }^{89}$ D. Tsionou, ${ }^{4, j j}$ G. Tsipolitis, ${ }^{9}$ S. Tsiskaridze, ${ }^{11}$ V. Tsiskaridze,${ }^{47}$ E. G. Tskhadadze, ${ }^{50 a}$ I. I. Tsukerman,${ }^{94}$ V. Tsulaia, ${ }^{14}$ J.-W. Tsung, ${ }^{20}$ S. Tsuno, ${ }^{64}$ D. Tsybychev, ${ }^{147}$ A. Tua ${ }^{138}$ A. Tudorache, ${ }^{25 a}$ V. Tudorache,${ }^{25 a}$ J. M. Tuggle,${ }^{30} \mathrm{M}$. Turala,${ }^{38} \mathrm{D}$. Turecek, ${ }^{126}$ I. Turk Cakir, ${ }^{3 \mathrm{e}}$ E. Turlay, ${ }^{104}$ R. Turra, ${ }^{88 a, 88 \mathrm{~b}}$ P. M. Tuts, ${ }^{34}$ A. Tykhonov, ${ }^{73}$ M. Tylmad,${ }^{145 a, 145 b}$ M. Tyndel, ${ }^{128}$ G. Tzanakos, ${ }^{8}$ K. Uchida, ${ }^{20}$ I. Ueda, ${ }^{154}$ R. Ueno, ${ }^{28}$ M. Ugland,${ }^{13}$ M. Uhlenbrock, ${ }^{20}$ M. Uhrmacher, ${ }^{53}$ F. Ukegawa, ${ }^{159}$ G. Unal, ${ }^{29}$ A. Undrus, ${ }^{24}$ G. Unel, ${ }^{162}$ Y. Unno, ${ }^{64}$ D. Urbaniec,${ }^{34}$ G. Usai, ${ }^{7}$ M. Uslenghi,${ }^{118 a, 118 b}$ L. Vacavant, ${ }^{82}$ V. Vacek, ${ }^{126}$ B. Vachon, ${ }^{84}$ S. Vahsen, ${ }^{14}$ J. Valenta, ${ }^{124}$ P. Valente, ${ }^{131 a}$ S. Valentinetti, ${ }^{19 a, 19 b}$ S. Valkar, ${ }^{125}$ E. Valladolid Gallego, ${ }^{166}$ S. Vallecorsa, ${ }^{151}$ J. A. Valls Ferrer, ${ }^{166}$ H. van der Graaf, ${ }^{104}$ E. van der Kraaij, ${ }^{104}$ R. Van Der Leeuw, ${ }^{104}$ E. van der Poel, ${ }^{104}$ D. van der Ster ${ }^{29}$ N. van Eldik, ${ }^{29}$ P. van Gemmeren, ${ }^{5}$ I. van Vulpen, ${ }^{104}$ M. Vanadia, ${ }^{98}$ W. Vandelli, ${ }^{29}$ A. Vaniachine,${ }^{5}$ P. Vankov, ${ }^{41}$ F. Vannucci, ${ }^{77}$ R. Vari, ${ }^{131 a}$ T. Varol, ${ }^{83}$ D. Varouchas, ${ }^{14}$ A. Vartapetian, ${ }^{7}$ K. E. Varvell ${ }^{149}$ V. I. Vassilakopoulos, ${ }^{55}$ F. Vazeille, ${ }^{33}$ T. Vazquez Schroeder, ${ }^{53}$ G. Vegni, ${ }^{88 a, 88 b}$ J. J. Veillet, ${ }^{114}$ F. Veloso, ${ }^{123 a}$ R. Veness,${ }^{29}$ S. Veneziano, ${ }^{131 a}$ A. Ventura, ${ }^{71 a, 71 b}$ D. Ventura,${ }^{83}$ M. Venturi, ${ }^{47}$ N. Venturi, ${ }^{157}$ V. Vercesi, ${ }^{118 \mathrm{a}}$ M. Verducci, ${ }^{137}$ W. Verkerke, ${ }^{104}$ J. C. Vermeulen, ${ }^{104}$ A. Vest,${ }^{43}$ M. C. Vetterli, ${ }^{141, \mathrm{e}}$ 
I. Vichou, ${ }^{164}$ T. Vickey, ${ }^{144 b, k k}$ O. E. Vickey Boeriu, ${ }^{144 b}$ G. H. A. Viehhauser,,${ }^{17}$ S. Viel, ${ }^{167}$ M. Villa, ${ }^{19 a, 19 b}$ M. Villaplana Perez, ${ }^{166}$ E. Vilucchi ${ }^{46}$ M. G. Vincter, ${ }^{28}$ E. Vinek,${ }^{29}$ V. B. Vinogradov ${ }^{63}$ M. Virchaux,${ }^{135, \text {,a }}$. Virzi, ${ }^{14}$ O. Vitells, ${ }^{171}$ M. Viti, ${ }^{41}$ I. Vivarelli, ${ }^{47}$ F. Vives Vaque, ${ }^{2}$ S. Vlachos, ${ }^{9}$ D. Vladoiu, ${ }^{97}$ M. Vlasak, ${ }^{126}$ A. Vogel, ${ }^{20}$ P. Vokac, ${ }^{16}$ G. Volpi, ${ }^{46}$ M. Volpi, ${ }^{85}$ G. Volpini, ${ }^{88 a}$ H. von der Schmitt, ${ }^{98}$ J. von Loeben, ${ }^{98}$ H. von Radziewski, ${ }^{47}$ E. von Toerne, ${ }^{20}$ V. Vorobel, ${ }^{125}$ V. Vorwerk, ${ }^{11}$ M. Vos, ${ }^{166}$ R. Voss, ${ }^{29}$ T. T. Voss, ${ }^{174}$ J. H. Vossebeld, ${ }^{72}$ N. Vranjes,${ }^{135}$ M. Vranjes Milosavljevic, ${ }^{104}$ V. Vrba, ${ }^{124}$ M. Vreeswijk, ${ }^{104}$ T. Vu Anh,${ }^{47}$ R. Vuillermet, ${ }^{29}$ I. Vukotic, ${ }^{14}$ W. Wagner, ${ }^{174}$ P. Wagner, ${ }^{119}$ H. Wahlen, ${ }^{174}$ S. Wahrmund, ${ }^{43}$ J. Wakabayashi, ${ }^{100}$ S. Walch, ${ }^{86}$ J. Walder,${ }^{70}$ R. Walker,${ }^{97}$ W. Walkowiak, ${ }^{140}$ R. Wall, ${ }^{175}$ P. Waller,${ }^{72} \mathrm{C}$. Wang, ${ }^{44} \mathrm{H}$. Wang, ${ }^{172} \mathrm{H}$. Wang, ${ }^{32 \mathrm{~b}, 11} \mathrm{~J}$. Wang, ${ }^{150} \mathrm{~J}$. Wang, ${ }^{54} \mathrm{R}$. Wang, ${ }^{102}$ S. M. Wang, ${ }^{150}$ T. Wang, ${ }^{20}$ A. Warburton, ${ }^{84}$ C. P. Ward,${ }^{27}$ M. Warsinsky, ${ }^{47}$ A. Washbrook, ${ }^{45}$ C. Wasicki, ${ }^{41}$ P. M. Watkins, ${ }^{17}$ A. T. Watson, ${ }^{17}$ I. J. Watson, ${ }^{149}$ M. F. Watson, ${ }^{17}$ G. Watts, ${ }^{137}$ S. Watts,${ }^{81}$ A. T. Waugh, ${ }^{149}$ B. M. Waugh, ${ }^{76}$ M. Weber ${ }^{128}$ M. S. Weber, ${ }^{16}$ P. Weber ${ }^{53}$ A. R. Weidberg, ${ }^{117}$ P. Weigell, ${ }^{98}$ J. Weingarten, ${ }^{53}$ C. Weiser, ${ }^{47}$ H. Wellenstein, ${ }^{22}$ P. S. Wells, ${ }^{29}$ T. Wenaus, ${ }^{24}$ D. Wendland, ${ }^{15}$ Z. Weng, ${ }^{150, x}$ T. Wengler, ${ }^{29}$ S. Wenig, ${ }^{29}$ N. Wermes ${ }^{20} \mathrm{M}$. Werner ${ }^{47} \mathrm{P}$. Werner, ${ }^{29} \mathrm{M}$. Werth, ${ }^{162} \mathrm{M}$. Wessels, ${ }^{57 \mathrm{a}} \mathrm{J}$. Wetter, ${ }^{160} \mathrm{C}$. Weydert, ${ }^{54} \mathrm{~K}$. Whalen, ${ }^{28}$ S. J. Wheeler-Ellis, ${ }^{162}$ A. White, ${ }^{7}$ M. J. White, ${ }^{85}$ S. White, ${ }^{121 \mathrm{a}, 121 \mathrm{~b}}$ S. R. Whitehead, ${ }^{117}$ D. Whiteson, ${ }^{162}$ D. Whittington, ${ }^{59}$ F. Wicek, ${ }^{114}$ D. Wicke, ${ }^{174}$ F. J. Wickens, ${ }^{128}$ W. Wiedenmann, ${ }^{172}$ M. Wielers,${ }^{128}$ P. Wienemann, ${ }^{20}$ C. Wiglesworth ${ }^{74}$ L. A. M. Wiik-Fuchs,${ }^{47}$ P. A. Wijeratne, ${ }^{76}$ A. Wildauer, ${ }^{166}$ M. A. Wildt, ${ }^{41, t}$ I. Wilhelm, ${ }^{125}$ H. G. Wilkens, ${ }^{29}$ J.Z. Will, ${ }^{97}$ E. Williams,${ }^{34}$ H. H. Williams, ${ }^{119}$ W. Willis,${ }^{34}$ S. Willocq,${ }^{83}$ J. A. Wilson, ${ }^{17}$ M. G. Wilson, ${ }^{142}$ A. Wilson, ${ }^{86}$ I. Wingerter-Seez,${ }^{4}$ S. Winkelmann, ${ }^{47}$ F. Winklmeier, ${ }^{29}$ M. Wittgen, ${ }^{142}$ M. W. Wolter, ${ }^{38}$ H. Wolters, ${ }^{123 a, i}$ W. C. Wong, ${ }^{40}$ G. Wooden, ${ }^{86}$ B. K. Wosiek, ${ }^{38}$ J. Wotschack, ${ }^{29}$ M. J. Woudstra ${ }^{81}$ K. W. Wozniak, ${ }^{38}$ K. Wraight, ${ }^{52}$ C. Wright,${ }^{52}$ M. Wright, ${ }^{52}$ B. Wrona, ${ }^{72}$ S. L. Wu, ${ }^{172} \mathrm{X}$. Wu, ${ }^{48} \mathrm{Y} . \mathrm{Wu},{ }^{32 \mathrm{~b}, \mathrm{~mm}}$ E. Wulf, ${ }^{34}$ B. M. Wynne, ${ }^{45}$ S. Xella, ${ }^{35}$ M. Xiao, ${ }^{135}$ S. Xie,${ }^{47}$ C. Xu, ${ }^{32 b, a a}$ D. Xu ${ }^{138}$ B. Yabsley, ${ }^{149}$ S. Yacoob, ${ }^{144 b}$ M. Yamada ${ }^{64}$ H. Yamaguchi, ${ }^{154}$ A. Yamamoto, ${ }^{64}$ K. Yamamoto, ${ }^{62}$ S. Yamamoto, ${ }^{154}$ T. Yamamura, ${ }^{154}$ T. Yamanaka, ${ }^{154}$ J. Yamaoka, ${ }^{44}$ T. Yamazaki, ${ }^{154}$ Y. Yamazaki, ${ }^{65}$ Z. Yan, ${ }^{21}$ H. Yang, ${ }^{86}$ U. K. Yang, ${ }^{81}$ Y. Yang, ${ }^{59}$ Z. Yang, ${ }^{145 a, 145 b}$ S. Yanush, ${ }^{90}$ L. Yao, ${ }^{32 a}$ Y. Yao, ${ }^{14}$ Y. Yasu, ${ }^{64}$ G. V. Ybeles Smit, ${ }^{129}$ J. Ye,${ }^{39}$ S. Ye,${ }^{24}$ M. Yilmaz,${ }^{3 c}$ R. Yoosoofmiya, ${ }^{122}$ K. Yorita, ${ }^{170}$ R. Yoshida, ${ }^{5}$ C. Young, ${ }^{142}$ C. J. Young, ${ }^{117}$ S. Youssef, ${ }^{21}$ D. Yu, ${ }^{24}$ J. Yu, ${ }^{7}$ J. Yu, ${ }^{111}$ L. Yuan, ${ }^{65}$ A. Yurkewicz, ${ }^{105}$ B. Zabinski, ${ }^{38}$ R. Zaidan, ${ }^{61}$ A. M. Zaitsev, ${ }^{127}$ Z. Zajacova, ${ }^{29}$ L. Zanello, ${ }^{131 a, 131 b}$ A. Zaytsev ${ }^{106}$ C. Zeitnitz, ${ }^{174}$ M. Zeman, ${ }^{124}$ A. Zemla,${ }^{38}$ C. Zendler ${ }^{20}$ O. Zenin,${ }^{127}$ T. Ženiš, ${ }^{143 a}$ Z. Zinonos, ${ }^{121 a}, 121 b$ S. Zenz ${ }^{14}$ D. Zerwas, ${ }^{114}$ G. Zevi della Porta, ${ }^{56}$ Z. Zhan, ${ }^{32 \mathrm{~d}}$ D. Zhang, ${ }^{32 \mathrm{~b}, 11}$ H. Zhang, ${ }^{87}$ J. Zhang, ${ }^{5}$ X. Zhang, ${ }^{32 \mathrm{~d}}$ Z. Zhang, ${ }^{114}$ L. Zhao, ${ }^{107}$ T. Zhao, ${ }^{137}$ Z. Zhao, ${ }^{32 b}$ A. Zhemchugov, ${ }^{63}$ J. Zhong, ${ }^{117}$ B. Zhou ${ }^{86}$ N. Zhou, ${ }^{162}$ Y. Zhou, ${ }^{150}$ C. G. Zhu, ${ }^{32 \mathrm{~d}}$ H. Zhu, ${ }^{41}$ J. Zhu, ${ }^{86}$ Y. Zhu, ${ }^{32 b}$ X. Zhuang, ${ }^{97}$ V. Zhuravlov, ${ }^{98}$ D. Zieminska, ${ }^{59}$ N. I. Zimin,${ }^{63}$ R. Zimmermann, ${ }^{20}$ S. Zimmermann ${ }^{20}$ S. Zimmermann, ${ }^{47}$ M. Ziolkowski, ${ }^{140}$ R. Zitoun, ${ }^{4}$ L. Živković,${ }^{34}$ V. V. Zmouchko, ${ }^{127, a}$ G. Zobernig, ${ }^{172}$ A. Zoccoli, ${ }^{19 a, 19 b}$ M. zur Nedden, ${ }^{15}$ V. Zutshi, ${ }^{105}$ and L. Zwalinski ${ }^{29}$

\title{
(ATLAS Collaboration)
}

\author{
${ }^{1}$ Physics Department, SUNY Albany, Albany, New York, USA \\ ${ }^{2}$ Department of Physics, University of Alberta, Edmonton, Alberta, Canada \\ ${ }^{3 a}$ Department of Physics, Ankara University, Ankara, Turkey \\ ${ }^{3 \mathrm{~b}}$ Department of Physics, Dumlupinar University, Kutahya, Turkey \\ ${ }^{3 \mathrm{c}}$ Department of Physics, Gazi University, Ankara, Turkey \\ ${ }^{3 \mathrm{~d}}$ Division of Physics, TOBB University of Economics and Technology, Ankara, Turkey \\ ${ }^{3 \mathrm{e}}$ Turkish Atomic Energy Authority, Ankara, Turkey \\ ${ }^{4}$ LAPP, CNRS/IN2P3 and Université de Savoie, Annecy-le-Vieux, France \\ ${ }^{5}$ High Energy Physics Division, Argonne National Laboratory, Argonne, Illinois, USA \\ ${ }^{6}$ Department of Physics, University of Arizona, Tucson, Arizona, USA \\ ${ }^{7}$ Department of Physics, The University of Texas at Arlington, Arlington, Texas, USA \\ ${ }^{8}$ Physics Department, University of Athens, Athens, Greece \\ ${ }^{9}$ Physics Department, National Technical University of Athens, Zografou, Greece \\ ${ }^{10}$ Institute of Physics, Azerbaijan Academy of Sciences, Baku, Azerbaijan \\ ${ }^{11}$ Institut de Física d'Altes Energies and Departament de Física de la Universitat Autònoma de Barcelona and ICREA, \\ Barcelona, Spain \\ ${ }^{12 a}$ Institute of Physics, University of Belgrade, Belgrade, Serbia \\ ${ }^{12 \mathrm{~b}}$ Vinca Institute of Nuclear Sciences, University of Belgrade, Belgrade, Serbia \\ ${ }^{13}$ Department for Physics and Technology, University of Bergen, Bergen, Norway
}


${ }^{14}$ Physics Division, Lawrence Berkeley National Laboratory and University of California, Berkeley, California, USA

${ }^{15}$ Department of Physics, Humboldt University, Berlin, Germany

${ }^{16}$ Albert Einstein Center for Fundamental Physics and Laboratory for High Energy Physics, University of Bern, Bern, Switzerland

${ }^{17}$ School of Physics and Astronomy, University of Birmingham, Birmingham, United Kingdom

${ }^{18 a}$ Department of Physics, Bogazici University, Istanbul, Turkey

${ }^{18 \mathrm{~b}}$ Division of Physics, Dogus University, Istanbul, Turkey

${ }^{18 c}$ Department of Physics Engineering, Gaziantep University, Gaziantep, Turkey

${ }^{18 \mathrm{~d} D e p a r t m e n t}$ of Physics, Istanbul Technical University, Istanbul, Turkey

${ }^{19 a}$ INFN Sezione di Bologna, Italy

${ }^{19 b}$ Dipartimento di Fisica, Università di Bologna, Bologna, Italy

${ }^{20}$ Physikalisches Institut, University of Bonn, Bonn, Germany

${ }^{21}$ Department of Physics, Boston University, Boston, Massachusetts, USA

${ }^{22}$ Department of Physics, Brandeis University, Waltham, Massachusetts, USA

${ }^{23 a}$ Universidade Federal do Rio De Janeiro COPPE/EE/IF, Rio de Janeiro, Brazil

${ }^{23 \mathrm{~b}}$ Federal University of Juiz de Fora (UFJF), Juiz de Fora, Brazil

${ }^{23 \mathrm{c}}$ Federal University of Sao Joao del Rei (UFSJ), Sao Joao del Rei, Brazil

${ }^{23 \mathrm{~d}}$ Instituto de Fisica, Universidade de Sao Paulo, Sao Paulo, Brazil

${ }^{24}$ Physics Department, Brookhaven National Laboratory, Upton, New York, USA

${ }^{25 a}$ National Institute of Physics and Nuclear Engineering, Bucharest, Romania

${ }^{25 \mathrm{~b}}$ University Politehnica Bucharest, Bucharest, Romania

${ }^{25 c}$ West University in Timisoara, Timisoara, Romania

${ }^{26}$ Departamento de Física, Universidad de Buenos Aires, Buenos Aires, Argentina

${ }^{27}$ Cavendish Laboratory, University of Cambridge, Cambridge, United Kingdom

${ }^{28}$ Department of Physics, Carleton University, Ottawa, Ontario, Canada

${ }^{29}$ CERN, Geneva, Switzerland

${ }^{30}$ Enrico Fermi Institute, University of Chicago, Chicago, Illinois, USA

${ }^{31 a}$ Departamento de Física, Pontificia Universidad Católica de Chile, Santiago, Chile

${ }^{31 \mathrm{~b}}$ Departamento de Física, Universidad Técnica Federico Santa María, Valparaíso, Chile

${ }^{32 a}$ Institute of High Energy Physics, Chinese Academy of Sciences, Beijing, China

${ }^{32 \mathrm{~b}}$ Department of Modern Physics, University of Science and Technology of China, Anhui, China

${ }^{32 \mathrm{c}}$ Department of Physics, Nanjing University, Jiangsu, China

${ }^{32 \mathrm{~d}}$ School of Physics, Shandong University, Shandong, China

${ }^{33}$ Laboratoire de Physique Corpusculaire, Clermont Université and Université Blaise Pascal and CNRS/IN2P3,

Aubiere Cedex, France

${ }^{34}$ Nevis Laboratory, Columbia University, Irvington, New York, USA

${ }^{35}$ Niels Bohr Institute, University of Copenhagen, Kobenhavn, Denmark

${ }^{36}$ INFN Gruppo Collegato di Cosenza, Italy

${ }^{36 \mathrm{~b}}$ Dipartimento di Fisica, Università della Calabria, Arcavata di Rende, Italy

${ }^{37}$ AGH University of Science and Technology, Faculty of Physics and Applied Computer Science, Krakow, Poland

${ }^{38}$ The Henryk Niewodniczanski Institute of Nuclear Physics, Polish Academy of Sciences, Krakow, Poland

${ }^{39}$ Physics Department, Southern Methodist University, Dallas, Texas, USA

${ }^{40}$ Physics Department, University of Texas at Dallas, Richardson, Texas, USA

${ }^{41}$ DESY, Hamburg and Zeuthen, Germany

${ }^{42}$ Institut für Experimentelle Physik IV, Technische Universität Dortmund, Dortmund, Germany

${ }^{43}$ Institut für Kern- und Teilchenphysik, Technical University Dresden, Dresden, Germany

${ }^{44}$ Department of Physics, Duke University, Durham, North Carolina, USA

${ }^{45}$ SUPA-School of Physics and Astronomy, University of Edinburgh, Edinburgh, United Kingdom

${ }^{46}$ INFN Laboratori Nazionali di Frascati, Frascati, Italy

${ }^{47}$ Fakultät für Mathematik und Physik, Albert-Ludwigs-Universität, Freiburg i.Br., Germany

${ }^{48}$ Section de Physique, Université de Genève, Geneva, Switzerland

${ }^{49 a}$ INFN Sezione di Genova, Italy

${ }^{49 \mathrm{~b}}$ Dipartimento di Fisica, Università di Genova, Genova, Italy

${ }^{50 a}$ E. Andronikashvili Institute of Physics, Tbilisi State University, Tbilisi, Georgia

${ }^{50 \mathrm{~b}}$ High Energy Physics Institute, Tbilisi State University, Tbilisi, Georgia

${ }^{51}$ II Physikalisches Institut, Justus-Liebig-Universität Giessen, Giessen, Germany

${ }^{52}$ SUPA-School of Physics and Astronomy, University of Glasgow, Glasgow, United Kingdom

${ }^{53}$ II Physikalisches Institut, Georg-August-Universität, Göttingen, Germany

${ }^{54}$ Laboratoire de Physique Subatomique et de Cosmologie, Université Joseph Fourier and CNRS/IN2P3 and Institut National Polytechnique de Grenoble, Grenoble, France

${ }^{55}$ Department of Physics, Hampton University, Hampton, Virginia, USA

${ }^{56}$ Laboratory for Particle Physics and Cosmology, Harvard University, Cambridge, Massachusetts, USA 
${ }^{57 a}$ Kirchhoff-Institut für Physik, Ruprecht-Karls-Universität Heidelberg, Heidelberg, Germany

${ }^{57 b}$ Physikalisches Institut, Ruprecht-Karls-Universität Heidelberg, Heidelberg, Germany

${ }^{57 \mathrm{c}}$ ZITI Institut für technische Informatik, Ruprecht-Karls-Universität Heidelberg, Mannheim, Germany

${ }^{58}$ Faculty of Applied Information Science, Hiroshima Institute of Technology, Hiroshima, Japan

${ }^{59}$ Department of Physics, Indiana University, Bloomington, Indiana, USA

${ }^{60}$ Institut für Astro- und Teilchenphysik, Leopold-Franzens-Universität, Innsbruck, Austria

${ }^{61}$ University of Iowa, Iowa City, Iowa, USA

${ }^{62}$ Department of Physics and Astronomy, Iowa State University, Ames, Iowa, USA

${ }^{63}$ Joint Institute for Nuclear Research, JINR Dubna, Dubna, Russia

${ }^{64}$ KEK, High Energy Accelerator Research Organization, Tsukuba, Japan

${ }^{65}$ Graduate School of Science, Kobe University, Kobe, Japan

${ }^{66}$ Faculty of Science, Kyoto University, Kyoto, Japan

${ }^{67}$ Kyoto University of Education, Kyoto, Japan

${ }^{68}$ Department of Physics, Kyushu University, Fukuoka, Japan

${ }^{69}$ Instituto de Física La Plata, Universidad Nacional de La Plata and CONICET, La Plata, Argentina

${ }^{70}$ Physics Department, Lancaster University, Lancaster, United Kingdom

${ }^{71 \mathrm{a}}$ INFN Sezione di Lecce, Italy

${ }^{71 \mathrm{~b}}$ Dipartimento di Matematica e Fisica, Università del Salento, Lecce, Italy

${ }^{72}$ Oliver Lodge Laboratory, University of Liverpool, Liverpool, United Kingdom

${ }^{73}$ Department of Physics, Jožef Stefan Institute and University of Ljubljana, Ljubljana, Slovenia

${ }^{74}$ School of Physics and Astronomy, Queen Mary University of London, London, United Kingdom

${ }^{75}$ Department of Physics, Royal Holloway University of London, Surrey, United Kingdom

${ }^{76}$ Department of Physics and Astronomy, University College London, London, United Kingdom

${ }^{77}$ Laboratoire de Physique Nucléaire et de Hautes Energies, UPMC and Université Paris-Diderot and CNRS/IN2P3, Paris, France

${ }^{78}$ Fysiska institutionen, Lunds universitet, Lund, Sweden

${ }^{79}$ Departamento de Fisica Teorica C-15, Universidad Autonoma de Madrid, Madrid, Spain

${ }^{80}$ Institut für Physik, Universität Mainz, Mainz, Germany

${ }^{81}$ School of Physics and Astronomy, University of Manchester, Manchester, United Kingdom

${ }^{82}$ CPPM, Aix-Marseille Université and CNRS/IN2P3, Marseille, France

${ }^{83}$ Department of Physics, University of Massachusetts, Amherst, Massachusetts, USA

${ }^{84}$ Department of Physics, McGill University, Montreal, Quebec, Canada

${ }^{85}$ School of Physics, University of Melbourne, Victoria, Australia

${ }^{86}$ Department of Physics, The University of Michigan, Ann Arbor, Michigan, USA

${ }^{87}$ Department of Physics and Astronomy, Michigan State University, East Lansing, Michigan, USA

${ }^{88}$ INFN Sezione di Milano, Italy

${ }^{88 \mathrm{~b}}$ Dipartimento di Fisica, Università di Milano, Milano, Italy

${ }^{89}$ B. I. Stepanov Institute of Physics, National Academy of Sciences of Belarus, Minsk, Republic of Belarus

${ }^{90}$ National Scientific and Educational Centre for Particle and High Energy Physics, Minsk, Republic of Belarus

${ }^{91}$ Department of Physics, Massachusetts Institute of Technology, Cambridge, Massachusetts, USA

${ }^{92}$ Group of Particle Physics, University of Montreal, Montreal, Quebec, Canada

${ }^{93}$ P. N. Lebedev Institute of Physics, Academy of Sciences, Moscow, Russia

${ }^{94}$ Institute for Theoretical and Experimental Physics (ITEP), Moscow, Russia

${ }^{95}$ Moscow Engineering and Physics Institute (MEPhI), Moscow, Russia

${ }^{96}$ Skobeltsyn Institute of Nuclear Physics, Lomonosov Moscow State University, Moscow, Russia

${ }^{97}$ Fakultät für Physik, Ludwig-Maximilians-Universität München, München, Germany

${ }^{98}$ Max-Planck-Institut für Physik (Werner-Heisenberg-Institut), München, Germany

${ }^{99}$ Nagasaki Institute of Applied Science, Nagasaki, Japan

${ }^{100}$ Graduate School of Science and Kobayashi-Maskawa Institute, Nagoya University, Nagoya, Japan

${ }^{101 a}$ INFN Sezione di Napoli, Italy

${ }^{101 \mathrm{~b}}$ Dipartimento di Scienze Fisiche, Università di Napoli, Napoli, Italy

${ }^{102}$ Department of Physics and Astronomy, University of New Mexico, Albuquerque, New Mexico, USA

${ }^{103}$ Institute for Mathematics, Astrophysics and Particle Physics, Radboud University Nijmegen/Nikhef, Nijmegen, Netherlands

${ }^{104}$ Nikhef National Institute for Subatomic Physics and University of Amsterdam, Amsterdam, Netherlands

${ }^{105}$ Department of Physics, Northern Illinois University, DeKalb, Illinois, USA

${ }^{106}$ Budker Institute of Nuclear Physics, SB RAS, Novosibirsk, Russia

${ }^{107}$ Department of Physics, New York University, New York, New York, USA

${ }^{108}$ Ohio State University, Columbus, Ohio, USA

${ }^{109}$ Faculty of Science, Okayama University, Okayama, Japan

${ }^{110}$ Homer L. Dodge Department of Physics and Astronomy, University of Oklahoma, Norman, Oklahoma, USA

${ }^{111}$ Department of Physics, Oklahoma State University, Stillwater, Oklahoma, USA

${ }^{112}$ Palacký University, RCPTM, Olomouc, Czech Republic 
${ }^{113}$ Center for High Energy Physics, University of Oregon, Eugene, Oregon, USA

${ }^{114}$ LAL, Université Paris-Sud and CNRS/IN2P3, Orsay, France

${ }^{115}$ Graduate School of Science, Osaka University, Osaka, Japan

${ }^{116}$ Department of Physics, University of Oslo, Oslo, Norway

${ }^{117}$ Department of Physics, Oxford University, Oxford, United Kingdom

${ }^{118 a}$ INFN Sezione di Pavia, Italy

${ }^{118 b}$ Dipartimento di Fisica, Università di Pavia, Pavia, Italy

${ }^{119}$ Department of Physics, University of Pennsylvania, Philadelphia, Pennsylvania, USA

${ }^{120}$ Petersburg Nuclear Physics Institute, Gatchina, Russia

${ }^{121 \text { a } I N F N ~ S e z i o n e ~ d i ~ P i s a, ~ I t a l y ~}$

${ }^{121 \mathrm{~b}}$ Dipartimento di Fisica E. Fermi, Università di Pisa, Pisa, Italy

${ }^{122}$ Department of Physics and Astronomy, University of Pittsburgh, Pittsburgh, Pennsylvania, USA

${ }^{123 a}$ Laboratorio de Instrumentacao e Fisica Experimental de Particulas-LIP, Lisboa, Portugal

${ }^{123 b}$ Departamento de Fisica Teorica y del Cosmos and CAFPE, Universidad de Granada, Granada, Spain

${ }^{124}$ Institute of Physics, Academy of Sciences of the Czech Republic, Praha, Czech Republic

${ }^{125}$ Faculty of Mathematics and Physics, Charles University in Prague, Praha, Czech Republic

${ }^{126}$ Czech Technical University in Prague, Praha, Czech Republic

${ }^{127}$ State Research Center Institute for High Energy Physics, Protvino, Russia

${ }^{128}$ Particle Physics Department, Rutherford Appleton Laboratory, Didcot, United Kingdom

${ }^{129}$ Physics Department, University of Regina, Regina, Saskatchewan, Canada

${ }^{130}$ Ritsumeikan University, Kusatsu, Shiga, Japan

${ }^{131 \mathrm{a}}$ INFN Sezione di Roma I, Italy

${ }^{131 \mathrm{~b}}$ Dipartimento di Fisica, Università La Sapienza, Roma, Italy

${ }^{132 a}$ INFN Sezione di Roma Tor Vergata, Italy

${ }^{132 \mathrm{~b}}$ Dipartimento di Fisica, Università di Roma Tor Vergata, Roma, Italy

${ }^{133 a}$ INFN Sezione di Roma Tre, Italy

${ }^{133 b}$ Dipartimento di Fisica, Università Roma Tre, Roma, Italy

${ }^{134 a}$ Faculté des Sciences Ain Chock, Réseau Universitaire de Physique des Hautes Energies-Université Hassan II, Casablanca, Morocco

${ }^{134 \mathrm{~b}}$ Centre National de l'Energie des Sciences Techniques Nucleaires, Rabat, Morocco

${ }^{134 \mathrm{c}}$ Faculté des Sciences Semlalia, Université Cadi Ayyad, LPHEA-Marrakech, Morocco

${ }^{134 \mathrm{~d}}$ Faculté des Sciences, Université Mohamed Premier and LPTPM, Oujda, Morocco

${ }^{134 \mathrm{e}}$ Faculté des sciences, Université Mohammed V-Agdal, Rabat, Morocco

${ }^{135}$ DSM/IRFU (Institut de Recherches sur les Lois Fondamentales de l'Univers), CEA Saclay (Commissariat a l'Energie Atomique), Gif-sur-Yvette, France

${ }^{136}$ Santa Cruz, Institute for Particle Physics, University of California Santa Cruz, Santa Cruz, California, USA

${ }^{137}$ Department of Physics, University of Washington, Seattle, Washington, USA

${ }^{138}$ Department of Physics and Astronomy, University of Sheffield, Sheffield, United Kingdom

${ }^{139}$ Department of Physics, Shinshu University, Nagano, Japan

${ }^{140}$ Fachbereich Physik, Universität Siegen, Siegen, Germany

${ }^{141}$ Department of Physics, Simon Fraser University, Burnaby, British Columbia, Canada

${ }^{142}$ SLAC National Accelerator Laboratory, Stanford, California, USA

${ }^{143 a}$ Faculty of Mathematics, Physics \& Informatics, Comenius University, Bratislava, Slovak Republic

${ }^{143 b}$ Department of Subnuclear Physics, Institute of Experimental Physics of the Slovak Academy of Sciences, Kosice, Slovak Republic

${ }^{144 a}$ Department of Physics, University of Johannesburg, Johannesburg, South Africa

${ }^{144 \mathrm{~b}}$ School of Physics, University of the Witwatersrand, Johannesburg, South Africa

${ }^{145}$ Department of Physics, Stockholm University, Sweden

${ }^{145 \mathrm{~b}}$ The Oskar Klein Centre, Stockholm, Sweden

${ }^{146}$ Physics Department, Royal Institute of Technology, Stockholm, Sweden

${ }^{147}$ Departments of Physics \& Astronomy and Chemistry, Stony Brook University, Stony Brook, New York, USA

${ }^{148}$ Department of Physics and Astronomy, University of Sussex, Brighton, United Kingdom

${ }^{149}$ School of Physics, University of Sydney, Sydney, Australia

${ }^{150}$ Institute of Physics, Academia Sinica, Taipei, Taiwan

${ }^{151}$ Department of Physics, Technion: Israel Institute of Technology, Haifa, Israel

${ }^{152}$ Raymond and Beverly Sackler School of Physics and Astronomy, Tel Aviv University, Tel Aviv, Israel

${ }^{153}$ Department of Physics, Aristotle University of Thessaloniki, Thessaloniki, Greece

${ }^{154}$ International Center for Elementary Particle Physics and Department of Physics, The University of Tokyo, Tokyo, Japan

${ }^{155}$ Graduate School of Science and Technology, Tokyo Metropolitan University, Tokyo, Japan

${ }^{156}$ Department of Physics, Tokyo Institute of Technology, Tokyo, Japan

${ }^{157}$ Department of Physics, University of Toronto, Toronto, Ontario, Canada

${ }^{158}$ TRIUMF, Vancouver, British Columbia, Canada 
${ }^{158 b}$ Department of Physics and Astronomy, York University, Toronto, Ontario, Canada

${ }^{159}$ Institute of Pure and Applied Sciences, University of Tsukuba,1-1-1 Tennodai,Tsukuba, Ibaraki 305-8571, Japan

${ }^{160}$ Science and Technology Center, Tufts University, Medford, Massachusetts, USA

${ }^{161}$ Centro de Investigaciones, Universidad Antonio Narino, Bogota, Colombia

${ }^{162}$ Department of Physics and Astronomy, University of California Irvine, Irvine, California, USA

${ }^{163 a}$ INFN Gruppo Collegato di Udine, Italy

${ }^{163 \mathrm{~b}}$ ICTP, Trieste, Italy

${ }^{163 \mathrm{c}}$ Dipartimento di Chimica, Fisica e Ambiente, Università di Udine, Udine, Italy

${ }^{164}$ Department of Physics, University of Illinois, Urbana, Illinois, USA

${ }^{165}$ Department of Physics and Astronomy, University of Uppsala, Uppsala, Sweden

${ }^{166}$ Instituto de Física Corpuscular (IFIC) and Departamento de Física Atómica, Molecular y Nuclear and Departamento de Ingeniería Electrónica and Instituto de Microelectrónica de Barcelona (IMB-CNM), University of Valencia and CSIC, Valencia, Spain

${ }^{167}$ Department of Physics, University of British Columbia, Vancouver, British Columbia, Canada

${ }^{168}$ Department of Physics and Astronomy, University of Victoria, Victoria, British Columbia, Canada

${ }^{169}$ Department of Physics, University of Warwick, Coventry, United Kingdom

${ }^{170}$ Waseda University, Tokyo, Japan

${ }^{171}$ Department of Particle Physics, The Weizmann Institute of Science, Rehovot, Israel

${ }^{172}$ Department of Physics, University of Wisconsin, Madison, Wisconsin, USA

${ }^{173}$ Fakultät für Physik und Astronomie, Julius-Maximilians-Universität, Würzburg, Germany

${ }^{174}$ Fachbereich C Physik, Bergische Universität Wuppertal, Wuppertal, Germany

${ }^{175}$ Department of Physics, Yale University, New Haven, Connecticut, USA

${ }^{176}$ Yerevan Physics Institute, Yerevan, Armenia

${ }^{177}$ Domaine scientifique de la Doua, Centre de Calcul CNRS/IN2P3, Villeurbanne Cedex, France

${ }^{\mathrm{a}}$ Deceased.

${ }^{\mathrm{b}}$ Also at Laboratorio de Instrumentacao e Fisica Experimental de Particulas-LIP, Lisboa, Portugal.

${ }^{\mathrm{c}}$ Also at Faculdade de Ciencias and CFNUL, Universidade de Lisboa, Lisboa, Portugal.

${ }^{\mathrm{d}}$ Also at Particle Physics Department, Rutherford Appleton Laboratory, Didcot, United Kingdom.

${ }^{\mathrm{e}}$ Also at TRIUMF, Vancouver, BC, Canada.

${ }^{\mathrm{f}}$ Also at Department of Physics, California State University, Fresno, CA, USA.

${ }^{\mathrm{g}}$ Also at Novosibirsk State University, Novosibirsk, Russia.

${ }^{\mathrm{h}}$ Also at Fermilab, Batavia, IL, USA.

${ }^{\mathrm{i}}$ Also at Department of Physics, University of Coimbra, Coimbra, Portugal.

${ }^{\mathrm{j}}$ Also at Department of Physics, UASLP, San Luis Potosi, Mexico.

${ }^{\mathrm{k}}$ Also at Università di Napoli Parthenope, Napoli, Italy.

${ }^{1}$ Also at Institute of Particle Physics (IPP), Canada.

${ }^{m}$ Also at Department of Physics, Middle East Technical University, Ankara, Turkey.

${ }^{\mathrm{n}}$ Also at Louisiana Tech University, Ruston, LA, USA.

${ }^{\circ}$ Also at Dep Fisica and CEFITEC of Faculdade de Ciencias e Tecnologia, Universidade Nova de Lisboa, Caparica, Portugal.

${ }^{\mathrm{p}}$ Also at Department of Physics and Astronomy, University College London, London, United Kingdom.

${ }^{\mathrm{q}}$ Also at Group of Particle Physics, University of Montreal, Montreal, QC, Canada.

${ }^{\mathrm{r}}$ Also at Department of Physics, University of Cape Town, Cape Town, South Africa.

${ }^{\mathrm{s}}$ Also at Institute of Physics, Azerbaijan Academy of Sciences, Baku, Azerbaijan.

${ }^{\mathrm{t} A l s o}$ at Institut für Experimentalphysik, Universität Hamburg, Hamburg, Germany.

${ }^{\mathrm{u}}$ Also at Manhattan College, New York, NY, USA.

${ }^{v}$ Also at School of Physics, Shandong University, Shandong, China.

${ }^{w}$ Also at CPPM, Aix-Marseille Université and CNRS/IN2P3, Marseille, France.

${ }^{\mathrm{x}}$ Also at School of Physics and Engineering, Sun Yat-sen University, Guanzhou, China.

${ }^{y}$ Also at Academia Sinica Grid Computing, Institute of Physics, Academia Sinica, Taipei, Taiwan.

${ }^{\mathrm{z}}$ Also at INFN Sezione di Roma I, Italy.

${ }^{\text {aa }}$ Also at DSM/IRFU (Institut de Recherches sur les Lois Fondamentales de l'Univers), CEA Saclay (Commissariat a l'Energie Atomique), Gif-sur-Yvette, France.

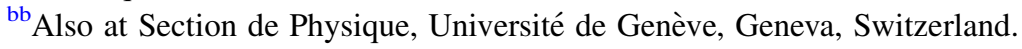

${ }^{\mathrm{cc}}$ Also at Departamento de Fisica, Universidade de Minho, Braga, Portugal.

${ }^{\mathrm{dd}}$ Also at Department of Physics and Astronomy, University of South Carolina, Columbia, SC, USA.

${ }^{e e}$ Also at Institute for Particle and Nuclear Physics, Wigner Research Centre for Physics, Budapest, Hungary.

${ }^{\mathrm{ff}}$ Also at California Institute of Technology, Pasadena, CA, USA. 
${ }^{g g}$ Also at Institute of Physics, Jagiellonian University, Krakow, Poland.

${ }^{\text {hh }}$ Also at LAL, Université Paris-Sud and CNRS/IN2P3, Orsay, France.

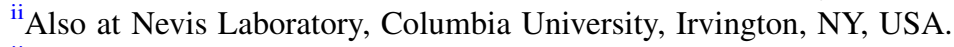

${ }^{\mathrm{jj}}$ Also at Department of Physics and Astronomy, University of Sheffield, Sheffield, United Kingdom.

${ }^{\mathrm{kk}}$ Also at Department of Physics, Oxford University, Oxford, United Kingdom.

${ }^{11}$ Also at Institute of Physics, Academia Sinica, Taipei, Taiwan.

${ }^{\mathrm{mm}}$ Also at Department of Physics, The University of Michigan, Ann Arbor, MI, USA. 